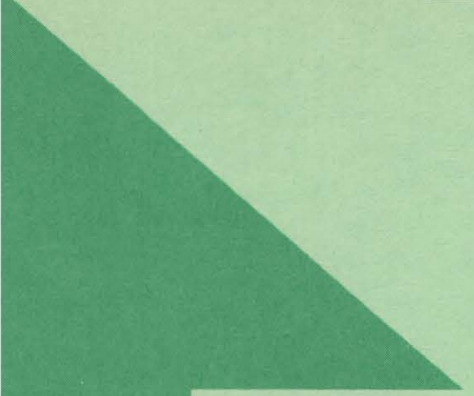

\title{
WATER TEMPERATURES IN THE LOWER COLUMBIA RIVER
}

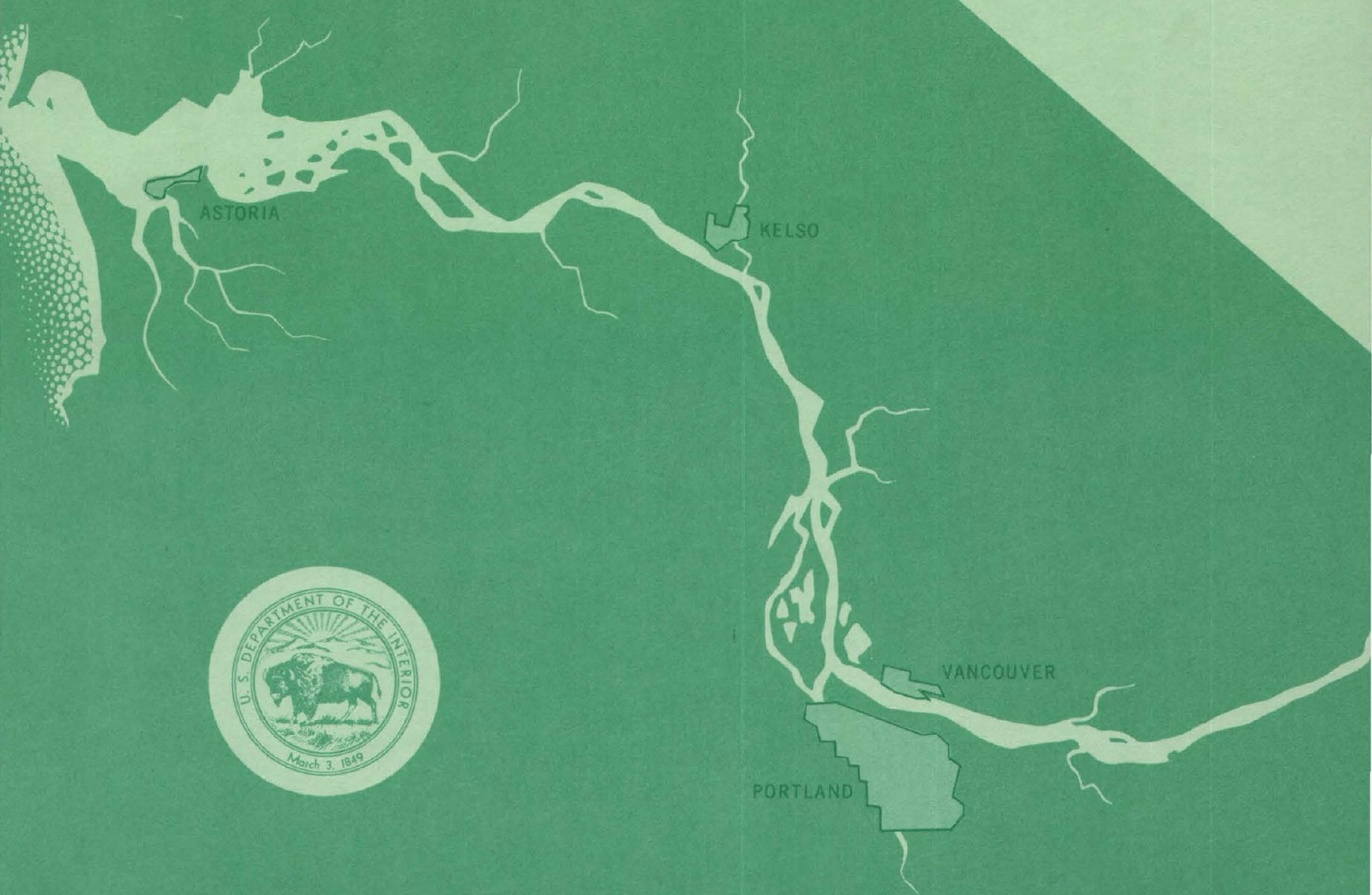

GEOLOGICAL SURVEY CIRCULAR 551 



\section{Water Temperatures in the Lower Columbia River}

GEOLOGICAL SURVEY CIRCULAR 551

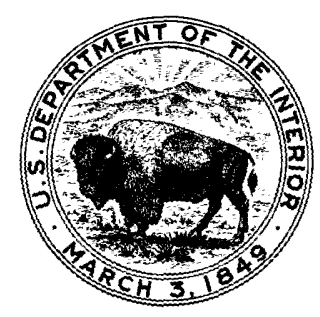


United States Department of the Interior STEWART L. UDALL, Secretary

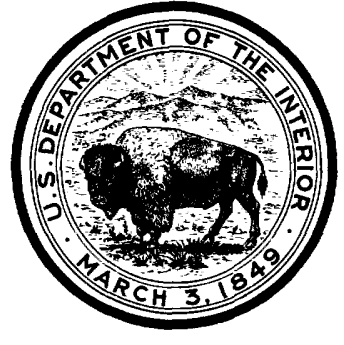

Geological Survey

William T. Pecora, Director

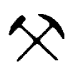




\section{CONTENTS}

$\begin{array}{cr}\text { Abstract } & \text { Page } \\ \text { Introduction } & 1 \\ \text { General background } & 1 \\ \text { Purpose and scope }- & 1 \\ \text { Acknowledgments } & 1 \\ \text { Daily water temperatures for 20 sites } & \end{array}$

Abstract

Monthly profiles of water temperature for August 1941 to July 1942

Explanation of profiles

Interpretation of profiles

Comparison with average conditions

Comparison with present conditions --.--

Conclusions

References
Page

2

2

10

10

10

15

16

\section{ILLUSTRATIONS}

Figure 1. Map showing study area and sites for which water temperatures are reported

2-7. Graphs of water-temperature profiles for lower Columbia River for-

2. August and September 1941

3. October and November 1941

4. December 1941 and January 1942

5. February and March 1942

6. April and May 1942

7. June and July 1942

8. Graph showing comparison of monthly water temperatures for 1941-42 with those for

1938-56 at Bonneville Dam

9. Graphs of monthly mean water temperatures at Bonneville Dam, 1938-66

10. Graphs showing correlation of water temperature at Bonneville Dam with air temperature and rate of flow (July)

11. Graphs showing correlation of water temperature at Bonneville Dam with air temperature and rate of flow (April)

\section{TABLES}

Table 1-6. Daily water temperatures of Columbia River, August 1941 to July 1942, at-

1. Warrendale, Oreg

2. Prindle, Wash

3. Washougal, Wash

4. Ellsworth, Wash

5. Vancouver, Wash

6. Kelley Point, Oreg

7. Daily water temperatures of Willamette River at Portland, Oreg. (Morrison Bridge),

August 1941 to July 1942

8. Daily water temperatures of Willamette River at Portland, Oreg. (U.S. Army Corps

of Engineers moorings), August 1941 to July 1942

9-14. Daily water temperatures of Columbia River, August 1941 to July 1942, at-

9. Willow-Bar, Wash

10. St. Helens, Oreg

11. Kalama, Wash

12. Longview, Wash

13. Stella, Wash

14. Eagle Cliff, Wash 
Table 15. Daily water temperatures of Columbia River at Wauna, Oreg., November 1940 to August 1942

16. Daily water temperatures of Columbia River at Cathlamet, Wash., September 1940 to July 1942

17. Daily water temperatures of Columbia River at Skamokawa, Wash., November $\mathbf{1 9 4 0}$ to July 1942

18. Daily water temperatures of Columbia River at Altoona, Wash., September 1940 to

19. Daily water temperatures of Columbia River at Astoria (Tongue Point), Oreg., 37 November 1940 to June 1942

20. Daily water temperatures of Columbia River at Fort Stevens, Oreg., August 1941 tc January 1942 and June, July 1942

21. Monthly maximum, minimum, and mean water temperatures of Columbia River at Bonneville Dam forebay 


\title{
WATER TEMPERATURES IN THE LOWER COLUMBIA RIVER
}

\author{
By A. M. MOoRE
}

\begin{abstract}
Daily observations of water temperature for 20 sites in the lower Columbia River are presented in tabular form and in profile form by months for the period August 1941 to July 1942. The profiles show minimum, mean (average), and maximum water temperatures for those months from river mile 142 to river mile 6.7. The data indicate that water temperature in the lower river trends upward from October to March and has no marked trend, or trends downward, during April to September.

Analysis of the water-temperature records at Bonneville Dam (river mile 146.1) for the 1938-66 period of record shows that the months from August 1941 to July 1942 are fairly representative of average conditions except for August and January, which are about $3^{\circ} \mathrm{F}$ above and $4^{\circ} \mathrm{F}$ below average, respectively. Analysis of the Bonneville Dam records also indicates that in the mid-1950's a man-caused change in watertemperature regimen occurred that resulted in higher temperatures at Bonneville Dam for 10 months of the year but no change in March and April. The changes for the other months ranged from $+0.5^{\circ}$ to $+2.0^{\circ} \mathrm{F}$. This means that average temperatures for the lower river are now somewhat warmer for most months than temperatures shown in the profiles.
\end{abstract}

\section{INTRODUCTION}

GENERAL BACKGROUND

Water temperatures in the Columbia River are becoming a matter of increasing interest and concern for several reasons. Water-quality standards are being drafted by the various States in the Columbia River basin, and a knowledge of water-temperature regimen throughout the basin is necessary in considering this phase of water quality. Furthermore, increasing use of the river by industry for cooling purposes can affect the water-temperature regimen as can the many reservoirs that have been or are being constructed. Proposed nuclear powerplants can affect water temperatures if river water is used as the coolant. Admittedly, a plant with a capacity of a million kilowatts will raise the temperature of the Columbia River by only a fraction of $1^{\circ} \mathrm{F}$ but several plants could have a significant cumulative effect.

Water-temperature records have been collected at hundreds of sites throughout the basin but very few in the tidal reach of the river below Bonneville Dam. In July 1966, the Northwest Water Resources Data Center began releasing weekly and monthly profiles of maximum and minimum water temperature for the main-stem Columbia and Snake Rivers to supply current information on water temperature. The Columbia River profile includes irformation for one site below Bonneville Dam, namely Beaver Army Terminal at river mile 53.5. The Corps of Engineers, U.S. Army, has in their files daily observations of water temparature at 18 sites in the main-stem Columbia River below Bonneville Dam and at two sites in the extreme lower Willamette River. These records, collected jointly by the Corps of Engineers and the U.S. Coast and Geodetic Survey, cover a period of a year or more in tho early 1940's.

\section{PURPOSE AND SCOPE}

Water-temperature records collected at 20 sites in the lower Columbia River basin (fig. 1) by the Corps of Engineers and the Coast and Geodetic Survey are presented in both tabular and profile form. The profiles show c'ata by months for the period August 1941 to Julr 1942. The report includes an analysis of the watertemperature records at Bonneville Dam for the period 1938-66 to determine the relationship of the profiles for 1941-42 to average corditions and whether there has been any change in temperature regimen in the lower river since 1942.

\section{ACKNOWLEDGMENTS}

The courtesy and cooperation of the Corps of Engineers and the Coast and Geodetic Survey in making these records availakle are gratefully acknowledged. 


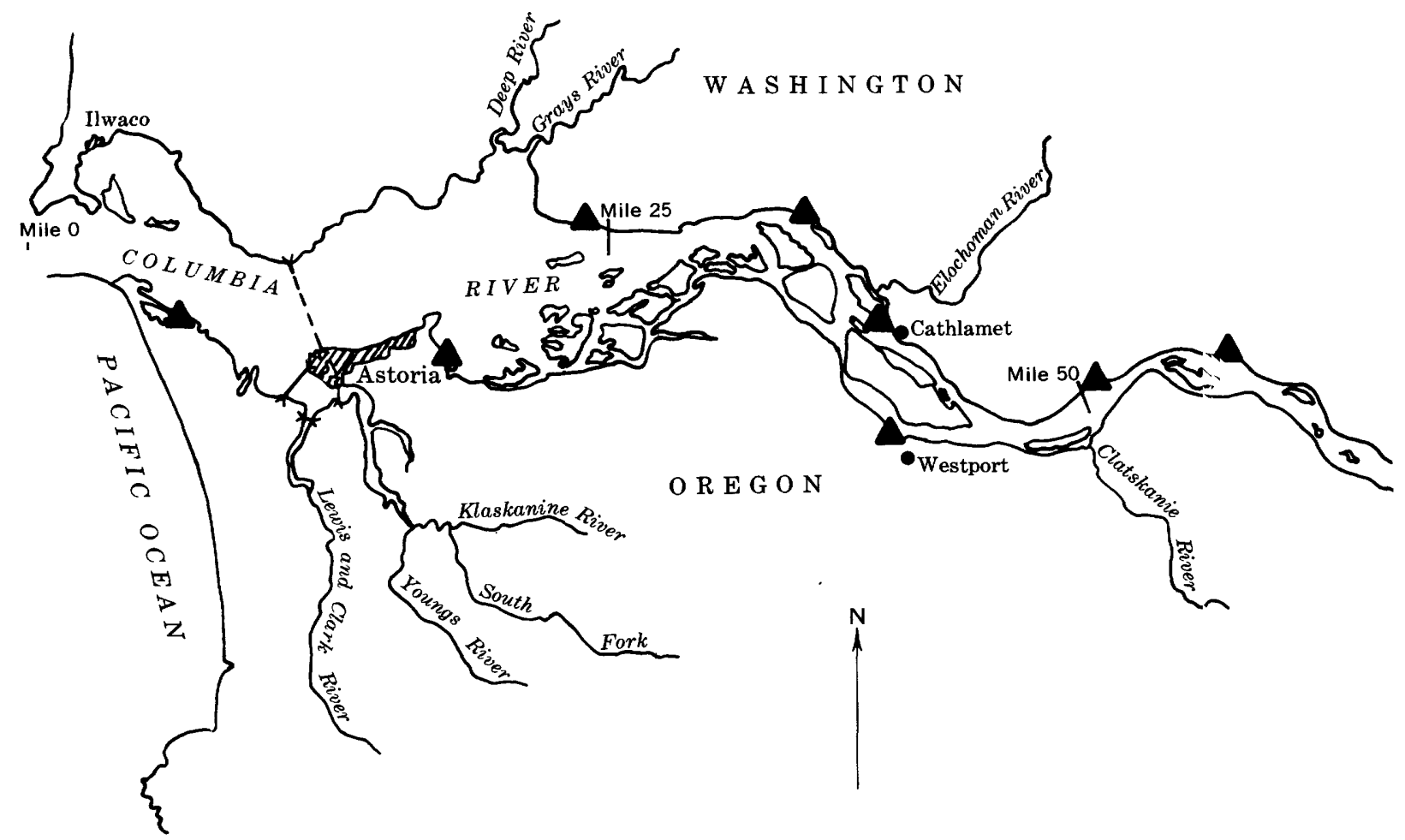

Water-temperature observation site

Figure 1.-Map showing study area and sites for which water temperatures are reported.

\section{DAILY WATER TEMPERATURES FOR 20 SITES}

Tables 1-20 list daily water temperatures for 18 sites in the main-stem Columbia River below Bonneville Dam and two sites in the lower Willamette River. All readings were made once daily with a hand thermometer, and no readings were made on some days (generally Sunday readings were omitted). The lack of readings on some days detracts only slightly from the utility of the records as there is little diurnal fluctuation of water temperature in the lower reaches of these rivers. Furthermore, the fluctuation that does occur is compensated for, as far as monthly means and extremes are concerned, because at most sites some readings were made in the morning and some in the afternoon. At some sites, observations were made using a centigrade (Celsius) thermometer but for this report were converted to the Fahrenheit scale. Some observations were recorded to tenths or halves of degrees but in all instances were rounded to the nearest degree.

\section{MONTHLY PROFILES OF WATER TEMPERATURE FOR AUGUST 1941 TO JULY 1942}

Profiles of monthly minimum, mean, and maximum water temperatures for the period $\mathrm{Au}$ gust 1941 to July 1942 are shown in figures 2-7.

\section{EXPLANATION OF PROFILES}

The profiles extend from Warrendale at river mile 142 to Fort Stevens at river mile 6.7. The monthly means and extremes of water temperature used in the profiles are shown at the bottom of tables 1-20. The 1941-42 period was selected because it covers 12 consecutive months for which data were available for each month at most of the sites.

The profiles generally were drawn as straight lines averaging the various points rather than as wavy lines passing through each point; the data are not complete or exact enough to define small variations between adjacent sites meaningfully. Data for a few sites may be affected by flow from a tributary that has not thoroughly mixed with the main-stem water. For 


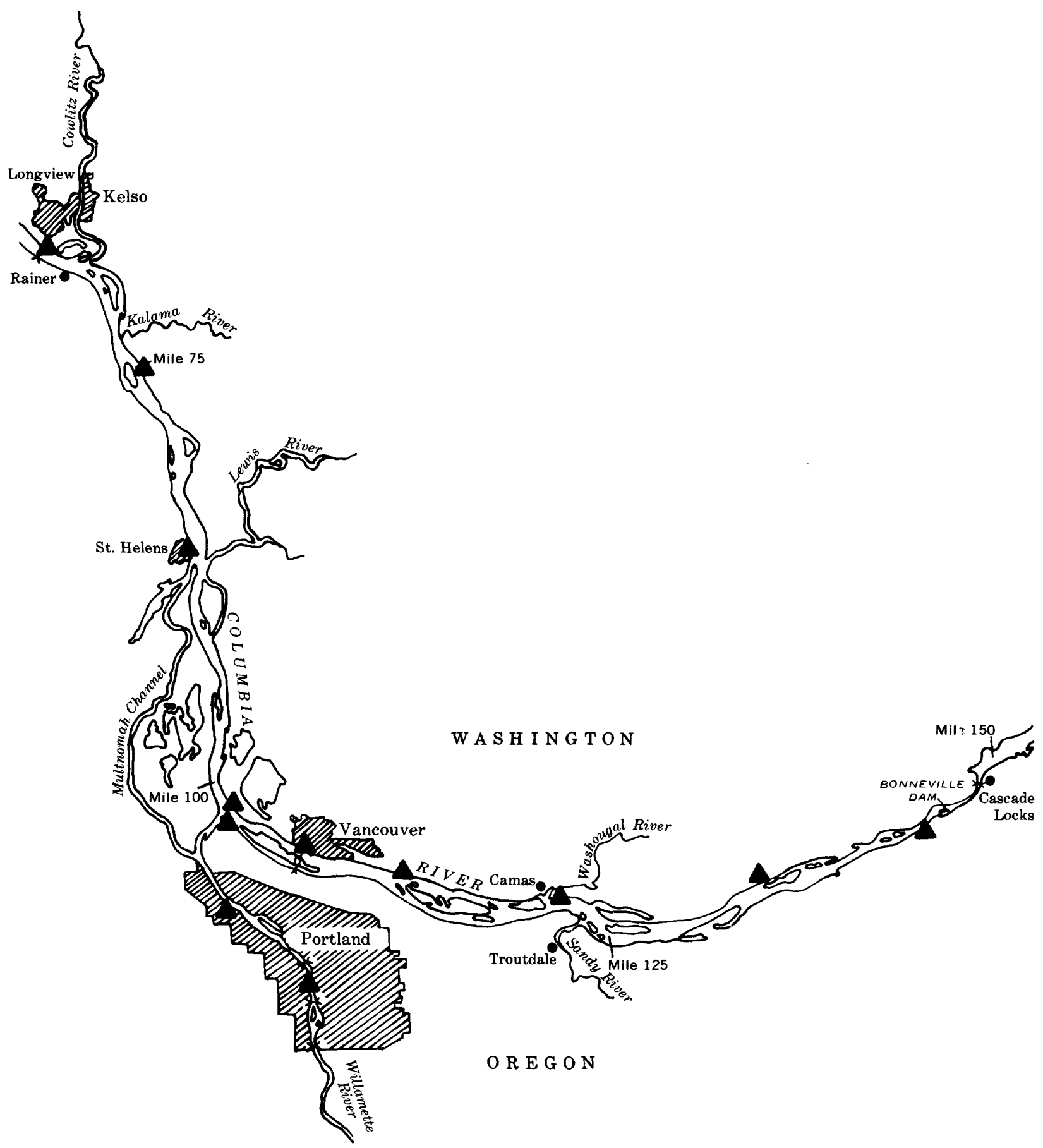

Figure 1.-Continued.

example, observations at Kelley Point, Oreg., are considerably higher than those upstream at Vancouver and those slightly downstream at Willow-Bar on the Washington side for the months of January to July 1942 and are slightly higher in 1 or 2 other months. Water from the Willamette River probably affected those readings. Willamette River water temperatures are shown on all the profiles. Note how closely the Kelley Point observations agree 


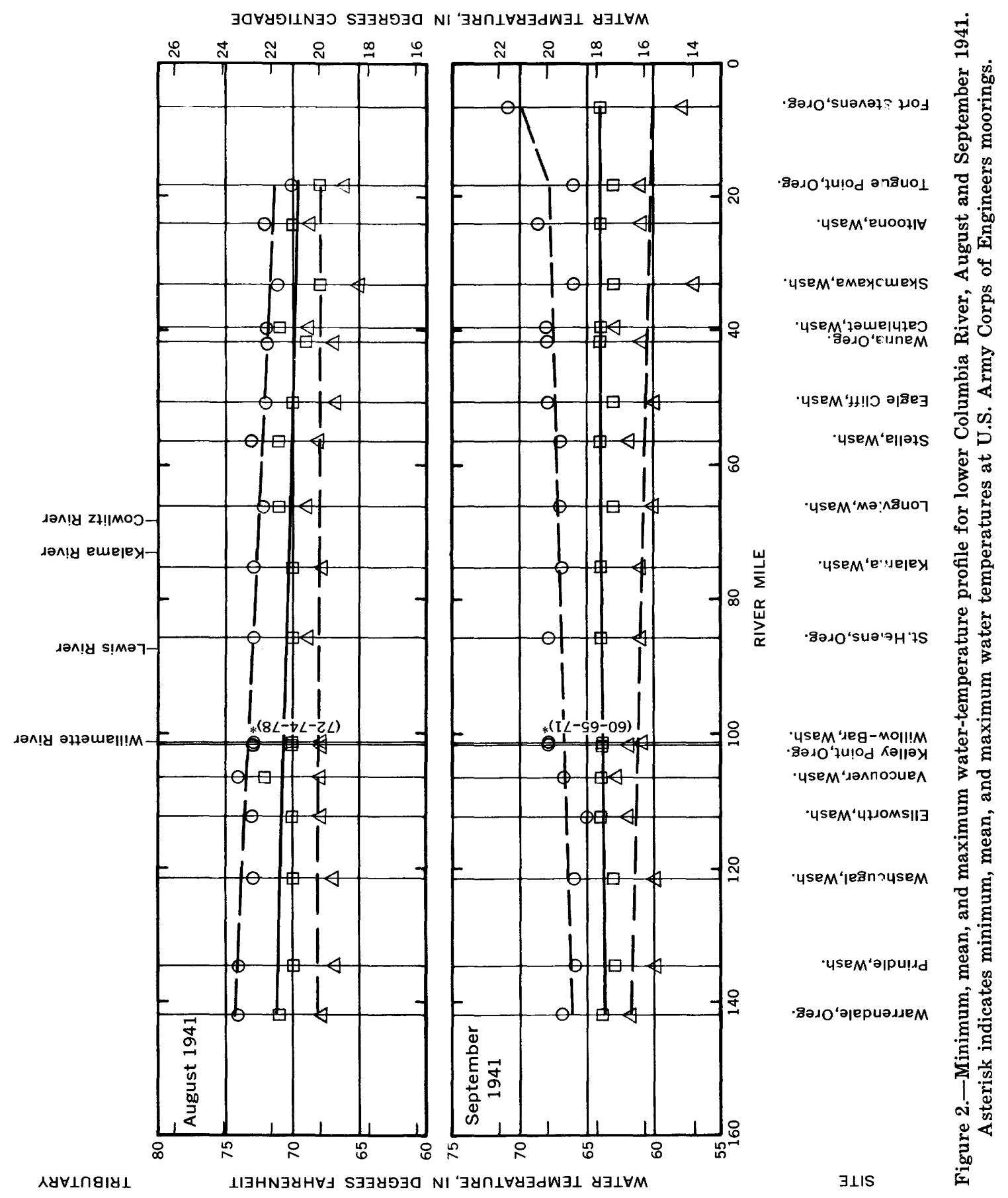



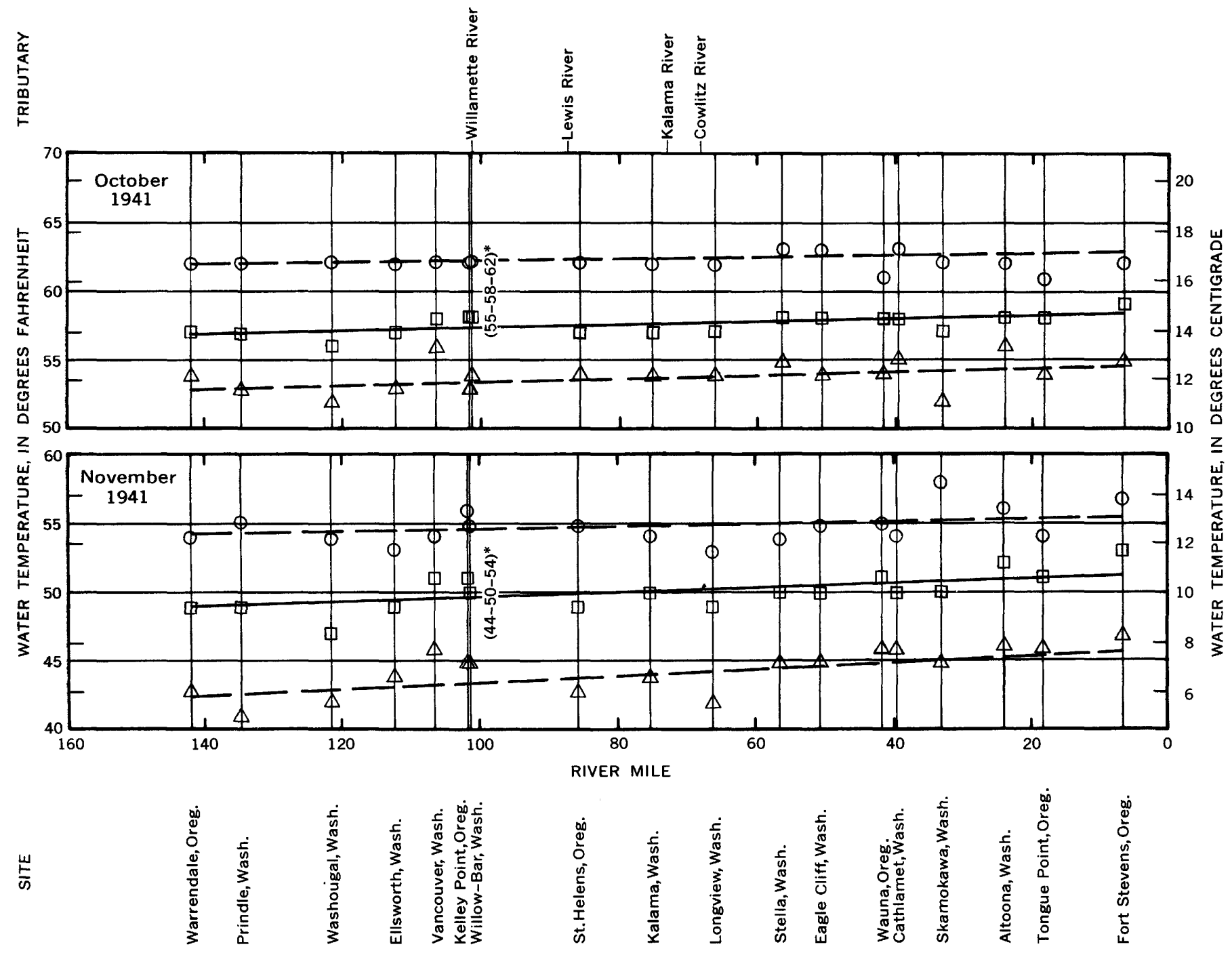

Figure 3.-Minimum, mean, and maximum water-temperature profile for lower Columbia River, October and November 1941. Asterisk indicates minimum, mean, and maximum water temperatures at Corps of Engineers moorings. 

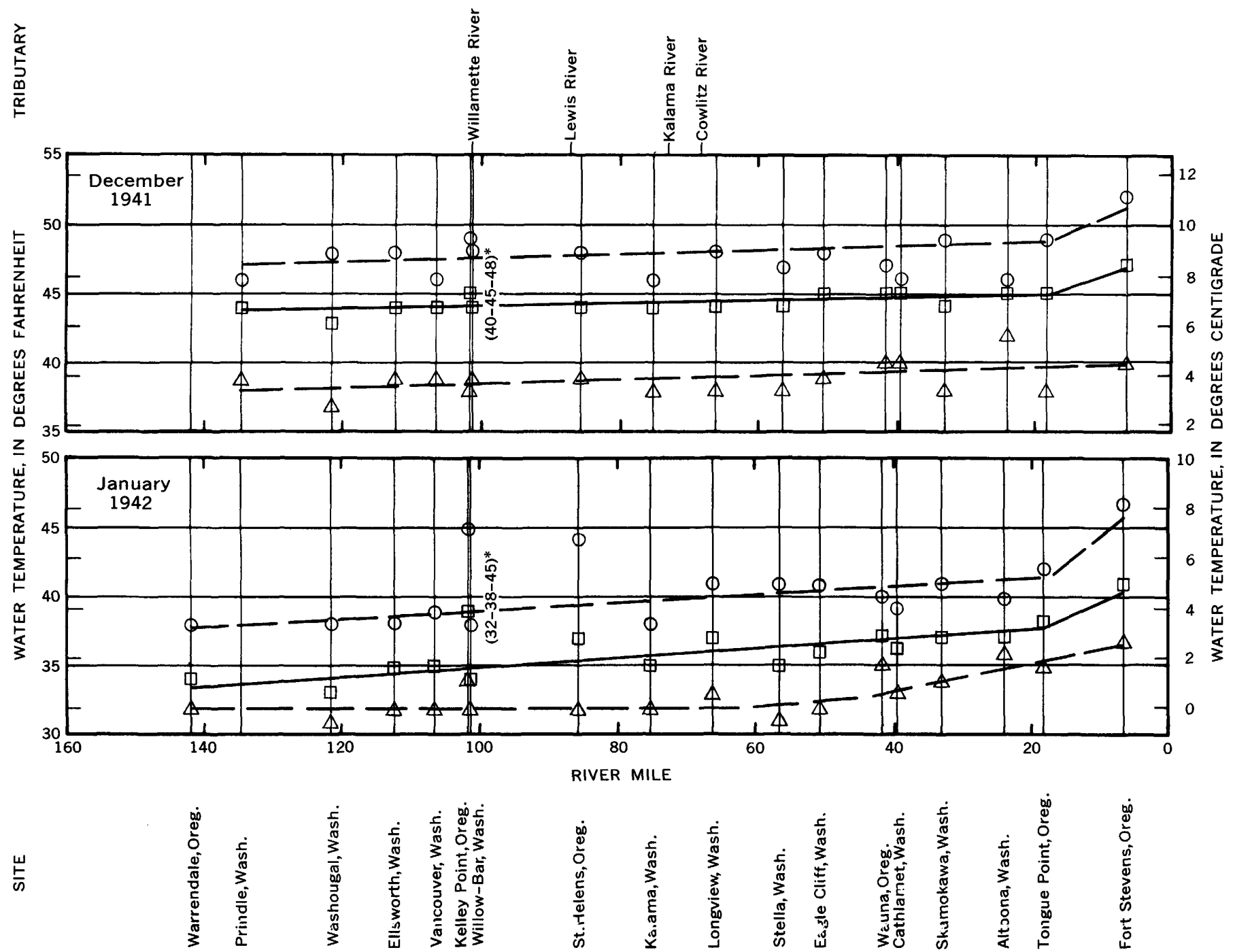

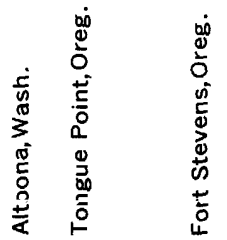

Figure 4.-Minimum, mean, and maximum water-temperature profile for lower Columbia River, December 1941 and January 1942. Asterisk indicates minimum, mean, and maximum water temperatures at Corps of Engineers moorings 

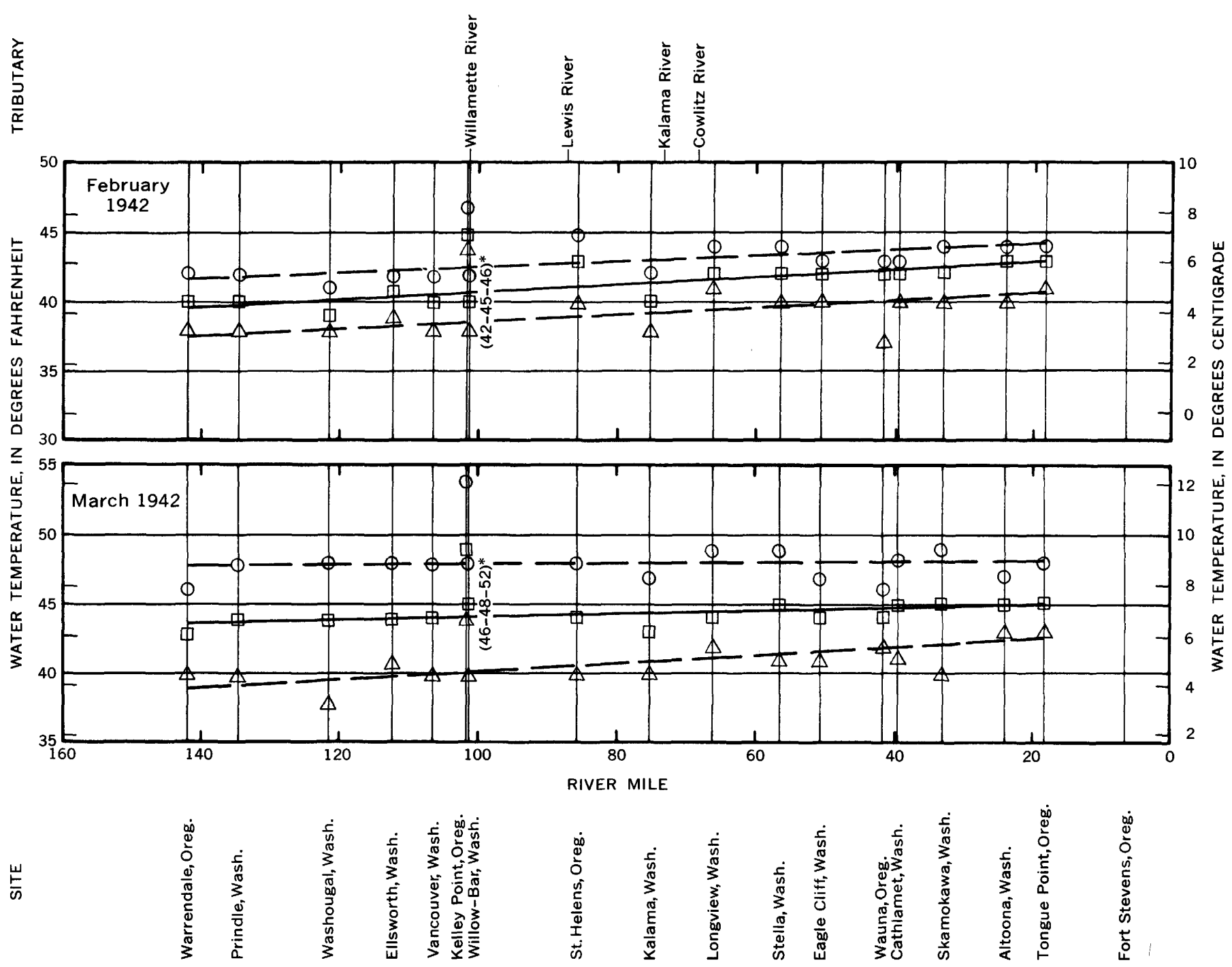

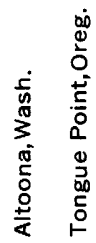

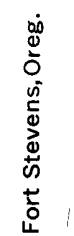

Figure 5.-Minimum, mean, and maximum water-temperature profile for lower Columbia River, February and March 1942. Asterisk indicates minimum, mean, and maximum water temperatures at Corps of Engineers moorings. 


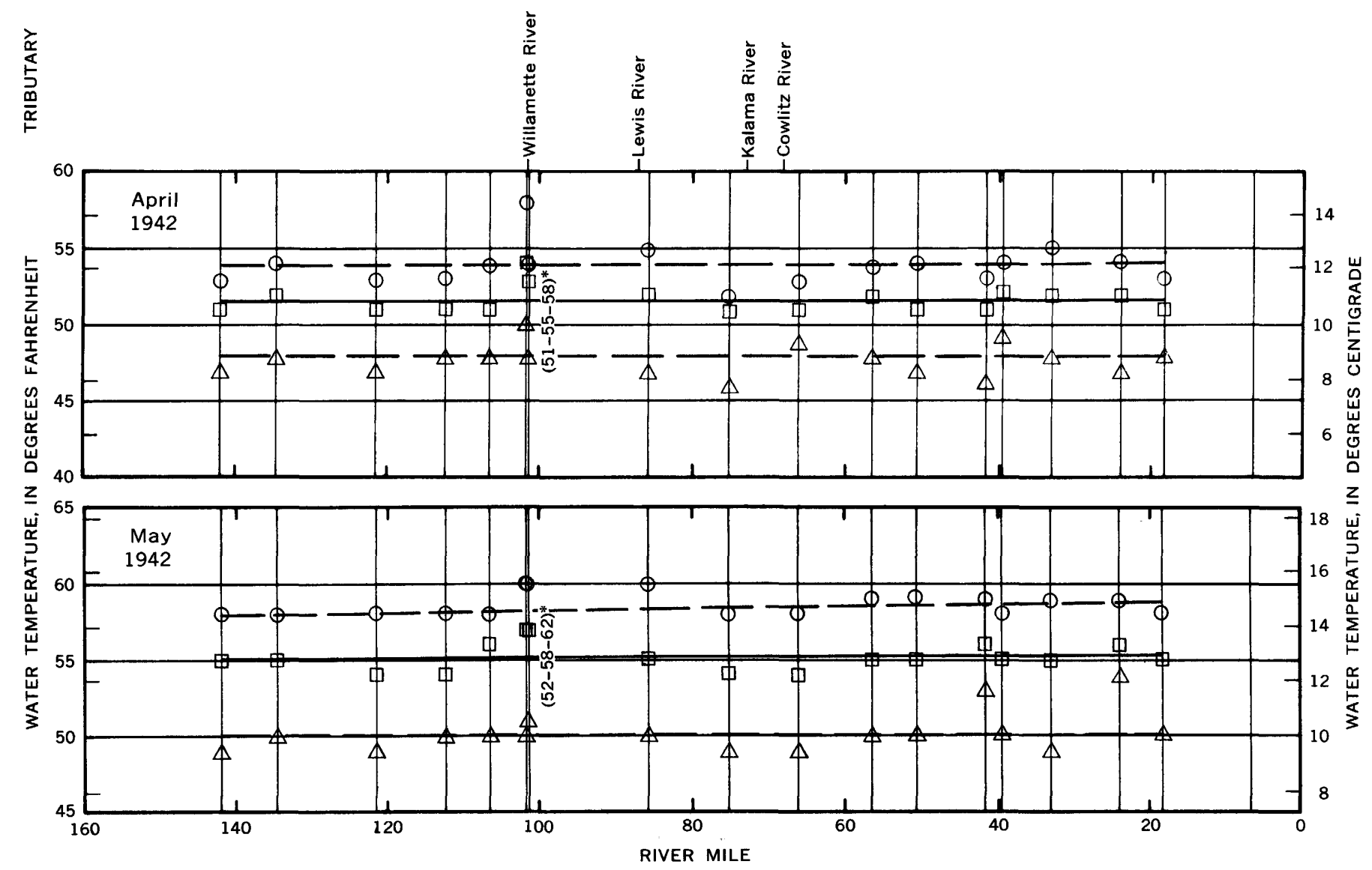

屴
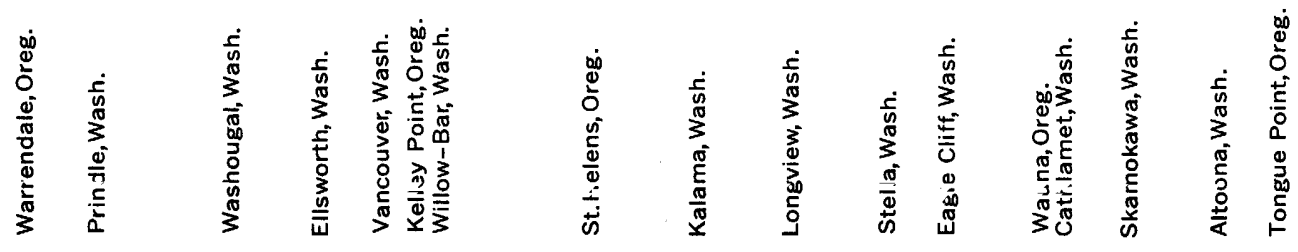

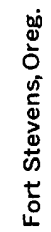

Figure 6.-Minimum, mean, and maximum water-temperature profile for lower Columbia River, April and May 1942. Asterisk indicates minimum, mean, and maximum water temperatures at Corps of Engineers moorings. 
WATER TEMPERATURE. IN DEGREES FAHRENHEIT

TRIBUTARY

SITE

胥

in

量.

依

․․․

:

萬

3.

క్․․․

\section{3}$$
\text { 今 }
$$$$
5
$$$$
\text { 茕 }
$$$$
\text { 造 }
$$$$
\text { ب. }
$$$$
\text { SITE }
$$

Warrendale, Oreg.

Prindle, Wash.

Washougal, Wash.

Ellsworth, Wash.

Vancouver, Wash.

Kelley Point, Oreg. Willow-Bar, Wash.

St. Helens, Oreg.

Kalama, Wash.

Longview, Wash.

Stella, Wash.

Eagle Cliff, Wash.

Wauna, Oreg.

Cathlamet, Wash.

Skamokawa, Wash.

Altoona, Wash.

Tongue Point, Oreg.

Fort Stevens, Oreg.

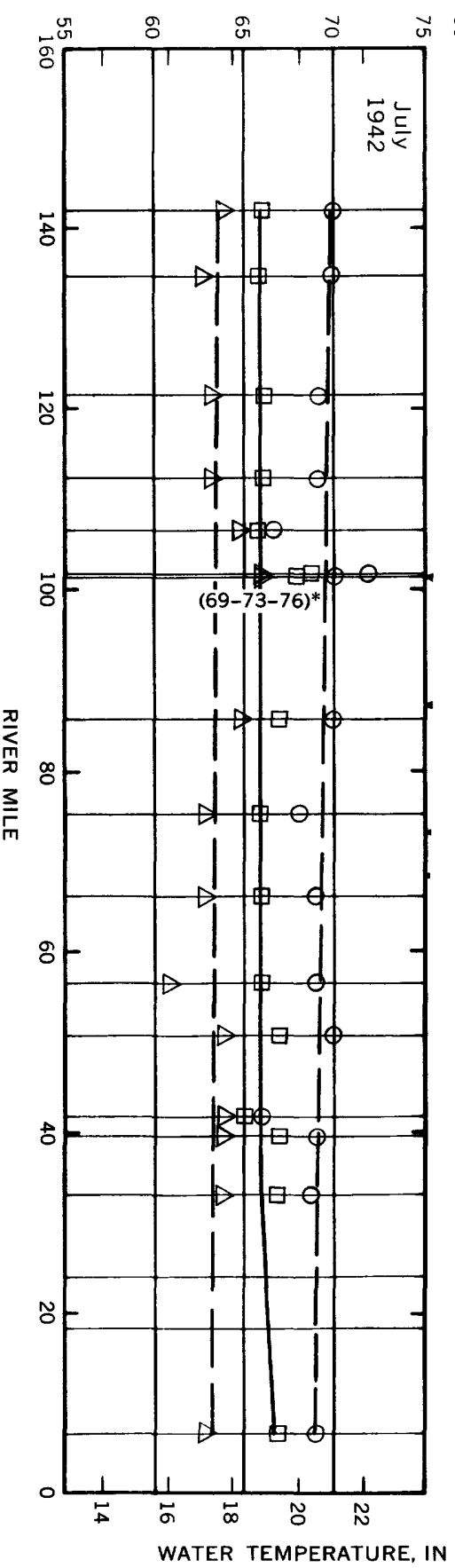

जे
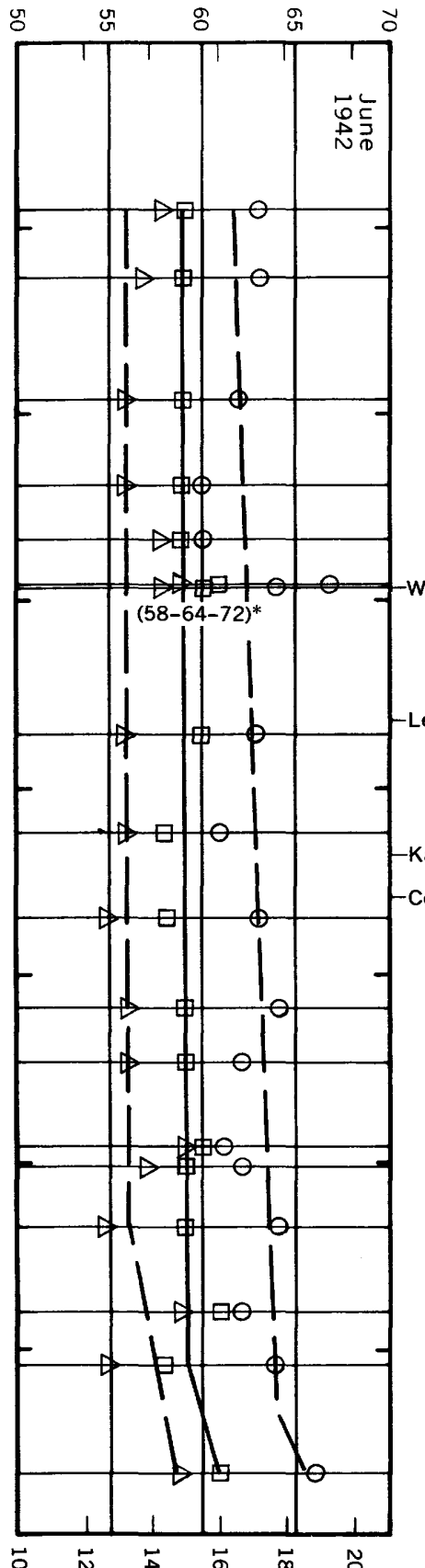

-Willamette River

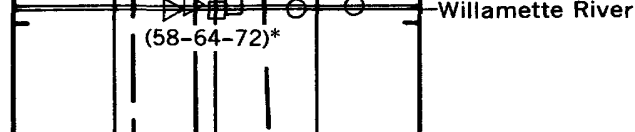

-Lewis River

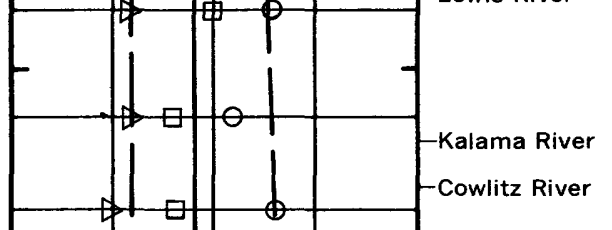


with Willamette River temperatures, especially during the winter months when Willamette River flows are high and Columbia River flows are low. For this reason the observations at Kelley Point were given no weight in drawing the profiles for most months.

\section{INTERPRETATION OF PROFILES}

The profiles indicate an upward trend in a downstream direction for October through March, and no pronounced trend or an upward trend during April through September. Meager data for the Fort Stevens site during the 12month period seem to indicate a rise in temperature in the last 15 miles above the mouth during winter and spring. Observations at that site may not represent true river-water temperatures because at high tide during the fall and winter, when the Columbia River is low, there probably is thorough mixing of fresh water with sea water which, at that time of the year, is warmer than the fresh water. Another possibility is that the observations at Fort Stevens were probably made in the smallboat basin where some water may remain for long periods before being flushed out, thereby providing a longer time for warming.

Questions that present themselves are: (1) How do these profiles compare with average conditions, and (2) are they representative of present conditions-that is, has there been a change in water-temperature regimen since 1942 ?

\section{Comparison With Average Conditions}

Because of the scarcity of long-term watertemperature data in the lower Columbia River, those obtained at Bonneville Dam, shown in table 21 , provide the best available means of comparing the 1941-42 data with average conditions. However, to make the comparison more meaningful, average maximum and minimum monthly temperatures rather than absolute maximum and minimum temperatures are used for 1938-56. The reason for using records for 1938-56 rather than $1938-66$ is that there appears to have been a change in the water-temperature regimen about 1956 . The data presented in figure 8 show that August 1941 was about $3^{\circ} \mathrm{F}$ above average and January 1942 was $4^{\circ} \mathrm{F}$ below average but the other months are within about $1^{\circ} \mathrm{F}$ of average conditions. Therefore, assuming that water-tempera- ture variations at Bonneville Dam are representative of those existing in the lower river, the profiles shown for the lower river are closely representative of average conditions for the period 1938-56 except for August 1941 and January 1942.

\section{Comparison With Present Conditions}

Because there are no longtime records at any of the sites used in the 1941-42 profiles, Bonneville Dam records also must be used to determine whether there has been any change in regimen since 1942. Figure 9 sl ows monthly mean water temperatures plotted against time for the 12 months of the year. ]'y inspection there appears to be an upward shift in water temperature after 1956 in all months except March and April, for which no shift is evident. The shift ranges from about $0.5^{\circ} \mathrm{F}$ in September to about $2^{\circ} \mathrm{F}$ in winter. The pcssibility that these shifts might be caused by natural phenomena such as a change in meteorologic conditions or deviations from average rates of flow was considered. To investigate these possibilities, water temperature was correlated with both air temperature and rate of flow.

There is a close correlation of monthly mean water temperature with monthly mean air temperature not only because air temperature affects water temperature but, more importantly, because both are affected by solar radiation. The correlation of water temperature with rate of flow is not so close, and for many months of the year the variatior in flow was so small that no correlation was found.

Although the multiple correlations were performed for each month of the year, only those for July and April (figs. 10 and 11) are shown here to illustrate methods used.

Air temperatures used were tro average of the monthly mean air temperatures at Wenatchee, Kennewick, Lewiston-Clarkston, and Bonneville. On figure $10 \mathrm{~A}$ the points representing July in the years 1938-56 are shown as circles, and those for 1957-66 are shown as triangles to provide easy visual identification of the data for each period. The fact that the points for $1957-66$ define a curve about $1^{\circ} \mathrm{F}$ to the right of the curve for 1938-56 indicates that the shift in mean water temperature was not caused by a change in meteorologic conditions. This still did not preclude the possibility 


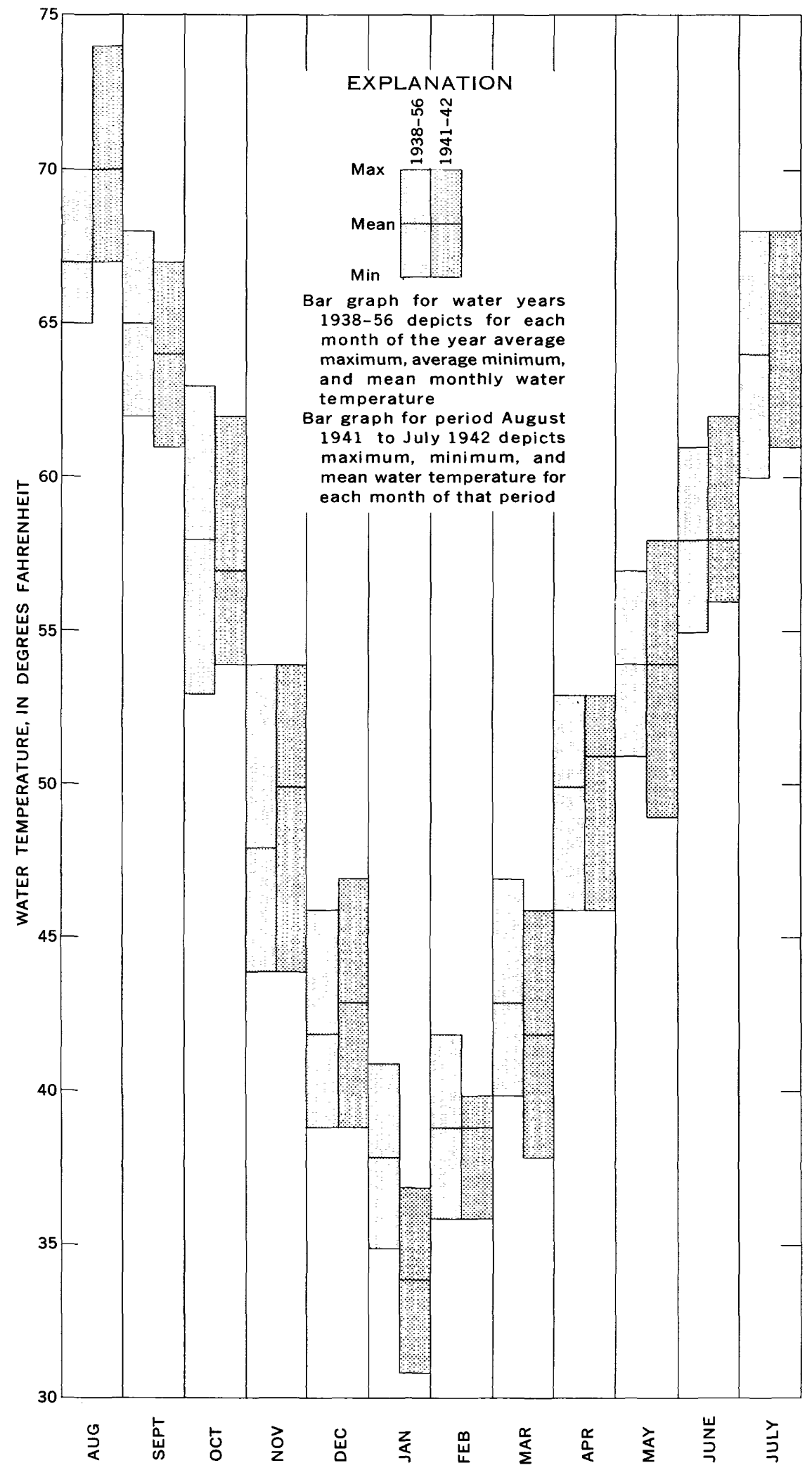

Figure 8.-Comparison of monthly water temperatures for 1941-42 with those for 1938-56 at Bonneville Dam. 

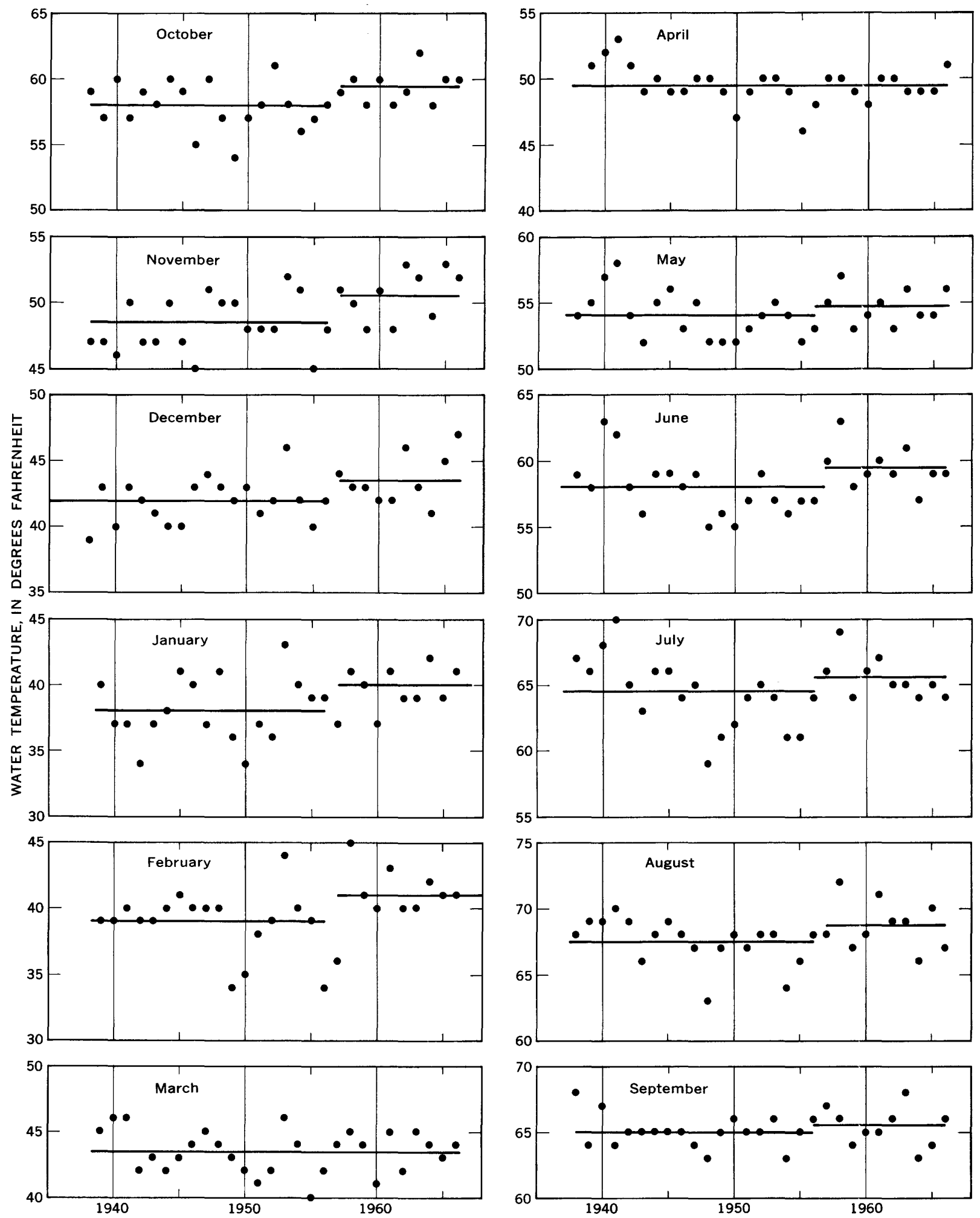

Figure 9.-Monthly mean water temperatures at Bonneville Dam, 1938-66. 


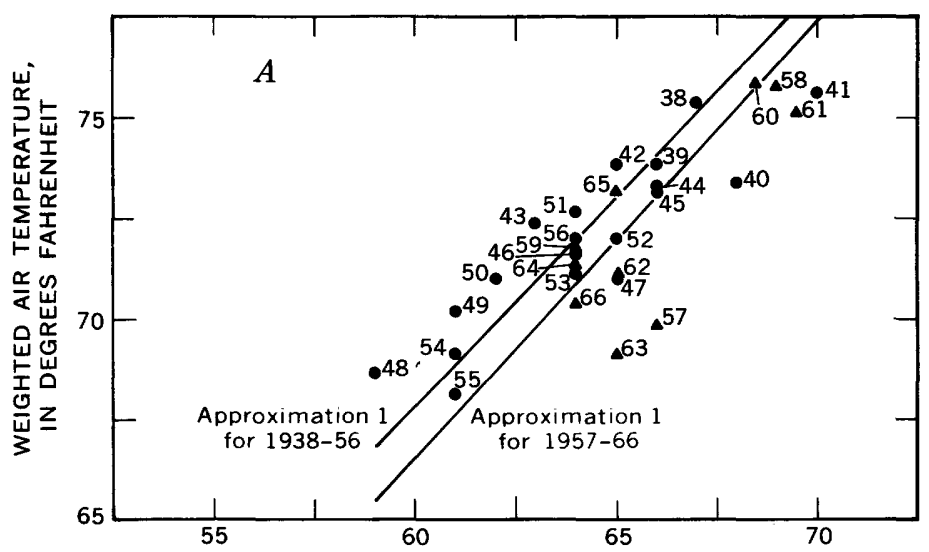

WATER TEMPERATURE, IN DEGREES FAHRENHEIT, AT BONNEVILLE DAM

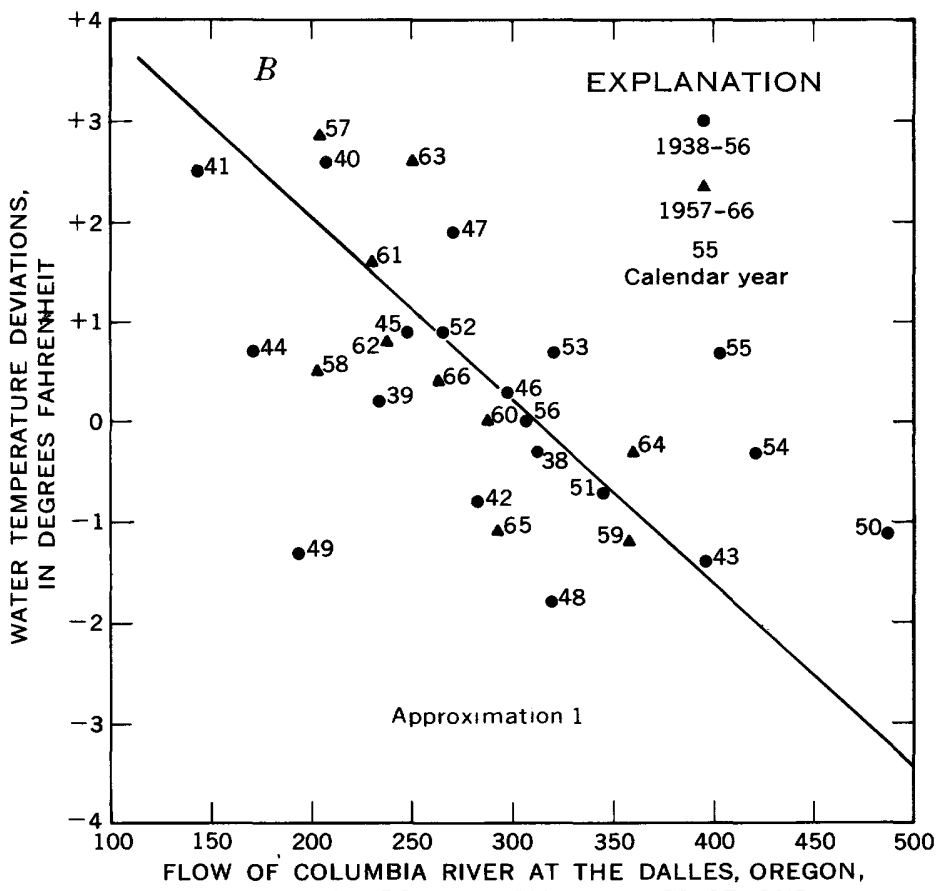

IN THOUSANDS OF CUBIC FEET PER SECOND

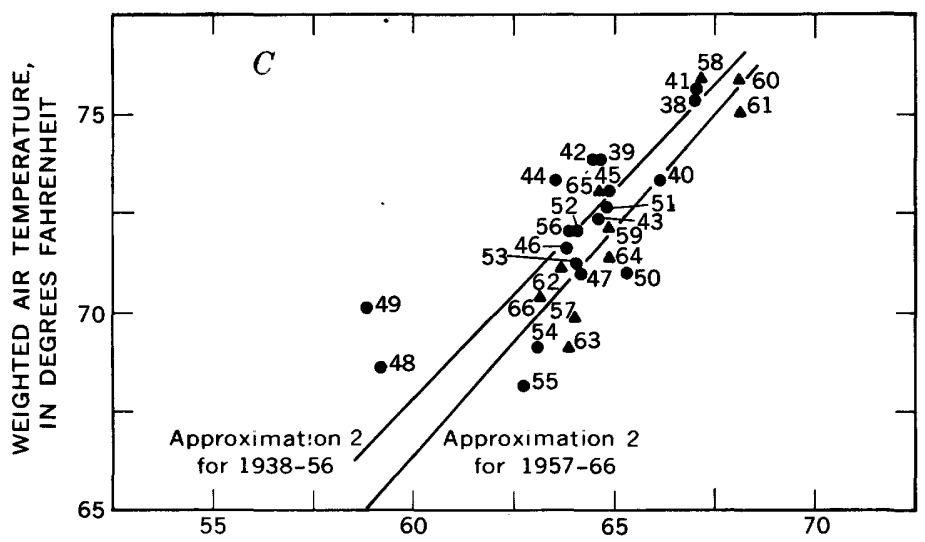

WATER TEMPERATURE, IN DEGREES FAHRENHEIT, AT BONNEVILLE DAM

Figure 10.-Correlation of water temperature at Bonneville Dam with air temperature and rate of flow (July). 


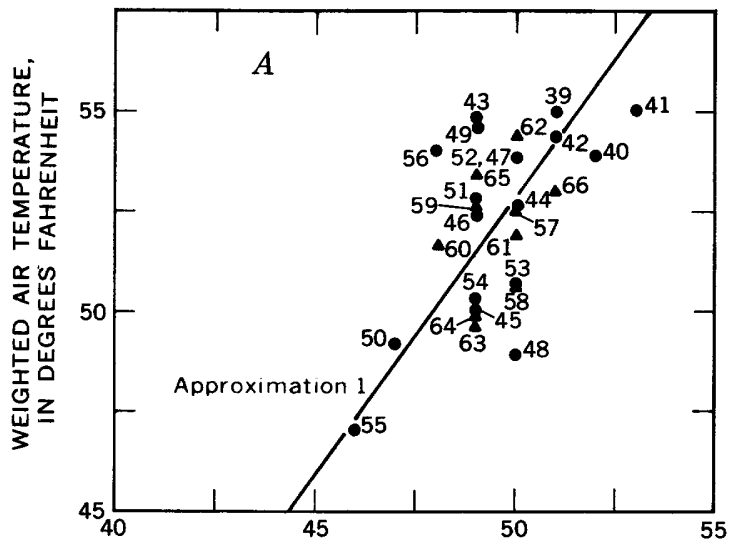

WATER TEMPERATURE, IN DEGREES FAHRENHEIT, AT BONNEVILLE DAM

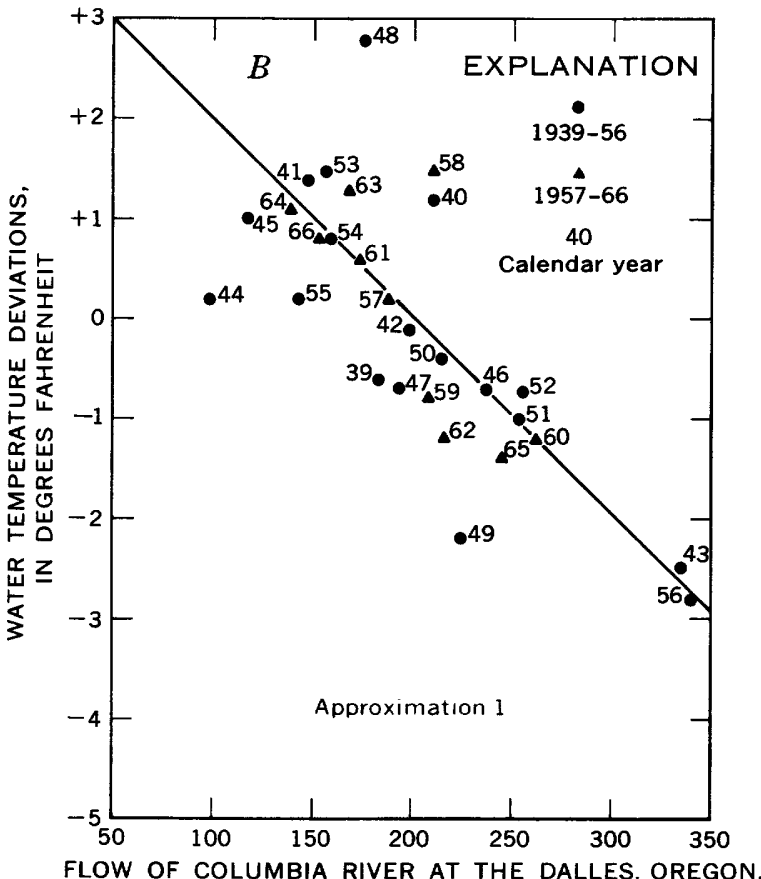

IN THOUSANDS OF CUBIC FEET PER SECOND

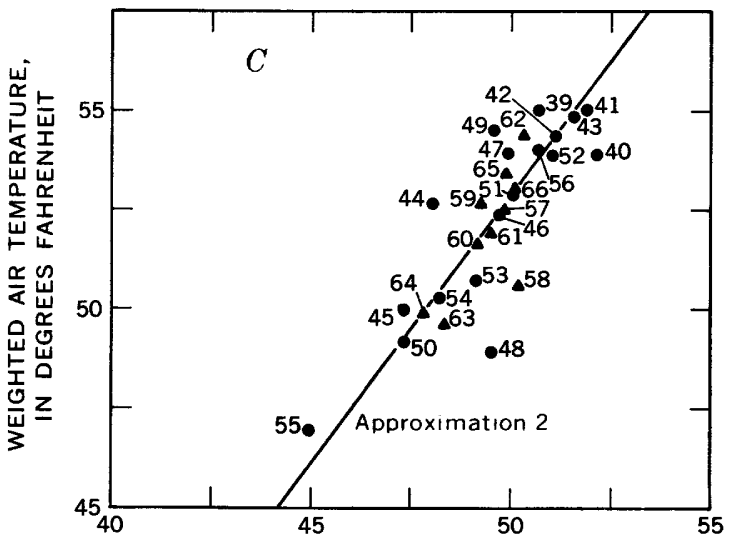

WATER TEMPERATURE, IN DEGREES FAHRENHEIT, AT BONNEVILLE DAM

Figure 11.-Correlation of water temperature at Bonneville Dam with air temperature and rate of flow (April). 
that rate of flow was causing the shift. Therefore, in figure $10 B$, flow was introduced as a second parameter by using the accepted graphic method of multiple correlation (Ezekiel and Fox, 1959, p. 254-277). Figure 10C indicates that, after taking both air temperature and flow into account, the points for 1957-66 still define a curve about $1^{\circ} \mathrm{F}$ to the right of that for 1938-56. This means that the $1^{\circ} \mathrm{F}$ upward shift in mean temperature shown by the July plot on figure 9 is not caused by a change in meteorologic conditions nor a change in the flow regimen and, therefore, represents a mancaused change in water-temperature regimen.

In figure $10 C$, the final plotting of points for 1952-56 might be taken to indicate that the shift in water temperature occurred as early as 1952. If the graphical correlation in figure 10 had been accomplished, assuming that the shift did occur in 1952, probably a shift of about $+1.5^{\circ} \mathrm{F}$ would have been found rather than $+1^{\circ} \mathrm{F}$. However, many of the multiple correlations for other months and the plotting in figure 9 for all the months of the year seem to indicate that the shift in temperature regimen occurred after 1956.

Figure 11 is similar to figure 10 but is for the month of April. The plot for April in figure 9 shows no change in water-temperature regimen. The graphical correlation in figure 11 checks figure 9 in this regard and further shows that meteorologic conditions and rate of flow were not masking a man-caused change in water temperature.

Results obtained from the graphical multiple correlations can be summarized as follows:

1. Shifts in water-temperature regimen, as shown by months in figure 9, are closely checked by the correlations.

2. These shifts, occurring in the middle 1950's probably are man caused, as the correlations indicate that they are not the result of changes in meteorologic conditions or flow regimen.

3. April through July were the only months where monthly flows varied enough from year to year to significantly affect the correlation between water and air temperatures.

\section{CONCLUSIONS}

First, the monthly profiles of water temperature for August 1941 to July 1942 indicate that, during the fall and winter, water temrorature of the Columbia River below Bonneville Dam trends upward in a downstream direction but during the spring and summer there is no pronounced trend or a downward trend. This regimen is probably attributable to the fact that above Bonneville Dam the Columbia River is exposed to a continental climate with cold winters and hot summers and that the lower river is exposed to a marine climate with moderate winters and summers.

Second, the profiles probably represent conditions that are within about $1^{\circ} \mathrm{F}$ of average for the period 1938-56 except those profiles for August 1941 and January 1942, which may be about $3^{\circ} \mathrm{F}$ higher and $4^{\circ} \mathrm{F}$ lower, respectively, than their 1938-56 averages.

Third, in the middle 1950's a change in water-temperature regimen occurred wlich apparently was man caused. The change resulted in an increase in water temperature at Bonneville Dam for all months except March and April, which were unchanged. The change was greatest, $1.5^{\circ}$ to $2^{\circ} \mathrm{F}$, during October through February. During the critically warm months of July to September the increase was only about $1^{\circ} \mathrm{F}$. It is understood that during the period 1954-61 operations of the Fanford Atomic Energy Plant at Richland, Wask, were increased considerably. This probably is the major cause of the change in water-temperature regimen at Bonneville Dam. However, concurrently with increased Hanford operations, interagency arrangements were made to provide for releases of cooler water from Lake Roosevelt to offset, at least in part, the warming effect of the atomic plant. Also, beginning with the 1959 water year, releases from the newly constructed Brownlee Reservoir supplied a cooling effect during summer, which amounted to as much as $2^{\circ} \mathrm{F}$ at Clarkston, Wash., 145 miles downstream (Moore, 1967, p. 36). Both Brownlee Reservoir and Lake Roosevelt supply a warming effect in fall and winter and a cooling effect in spring and summer. Lake Roose- 
velt had supplied this effect throughout practically the entire period of water-temperature record at Bonneville Dam, but in the middle 1950 's the cooling effect was intentionally increased as explained above. Therefore, the Hanford operations are believed to be the principal cause of the increase in water temperature at Bonneville Dam, but the temperature of releases from Lake Roosevelt and Brownlee Reservoir probably explain why the increase is only about $1^{\circ} \mathrm{F}$ in summer and is about $2^{\circ} \mathrm{F}$ in fall and winter. Construction of some "run-ofriver" reservoirs such as those above The Dalles and McNary Dams also may have had some effect on the temperature regimen, but this is believed to be relatively minor.
Fourth, the change in water-temperature regimen occurring in the middle 1950's at Bonneville Dam undoubtedly extends to the lower river, but probably the magnitude of the change diminishes in a downstream direction. The changes at Bonneville Dam, therefore, as shown in figure 9, cannot be used directly in determining how the 1941-42 prcfiles of water temperature compare with the 1957-66 monthly averages at Bonneville Dam. However, as far as the critical summer months are concerned, the July 1942 and September 1941 profiles probably are within about $1^{\circ} \mathrm{F}$ of the 1957-66 averages, and the profile for August 1941 should be somewhat less than $3^{\circ} \mathrm{F}$ above the 1957-66 average.

\section{REFERENCES}

Ezekiel, Mordecai, and Fox, Karl A., 1959, Methods of correlation and regression analysis: New York, John Wiley \& Sons, 548 p.
Moore, A. M., 1967, Correlation and aralysis of watertemperature data for Oregon streams: U.S. Geol. Survey Water-Supply Paper 1819-K, 53 p. 
TABLES 1-21 

Table 1.--Daily water temperatures of Columbia River at Warrendale, Oreg., August 1941 to July 1942

[Once-daily observations--mixed morning and afternoon readings at river mile 142 ]

\begin{tabular}{|c|c|c|c|c|c|c|c|c|c|c|c|c|}
\hline Day & August & September & October & November & De:ember & January & February & March & April & May & June & July \\
\hline 1 & & 67 & 62 & 54 & & 38 & 38 & 40 & 47 & 49 & 58 & 64 \\
\hline 2 & & 66 & 62 & - & & 35 & 38 & 40 & 48 & 50 & 58 & 64 \\
\hline 3 & & -- & 60 & 53 & & 33 & 38 & 40 & 49 & 50 & 58 & 64 \\
\hline 4 & & 66 & 60 & 53 & & 32 & 38 & 40 & 50 & 50 & 58 & 67 \\
\hline 5 & & 66 & 60 & 53 & & 32 & 40 & 40 & 50 & 52 & 58 & 67 \\
\hline 6 & & 65 & 58 & 52 & & $\frac{21}{32}$ & 39 & 41 & 50 & 53 & 59 & $\frac{11}{66}$ \\
\hline 7 & & 65 & 58 & 52 & & -- & 40 & 42 & 51 & 54 & 58 & 66 \\
\hline 8 & & 65 & 58 & 52 & & 32 & 40 & 42 & 52 & 54 & 60 & 66 \\
\hline 9 & & 65 & 58 & 52 & & 32 & 41 & 43 & 52 & 54 & 59 & 66 \\
\hline 10 & & 66 & 58 & 52 & & 32 & 41 & 43 & 52 & 54 & 59 & 66 \\
\hline 11 & 69 & -- & 57 & 52 & & 32 & 41 & 43 & -- & 54 & 58 & 65 \\
\hline 12 & 70 & 64 & 57 & 51 & & 32 & 41 & 44 & 53 & 55 & 59 & 64 \\
\hline 13 & 70 & 64 & 57 & 50 & & 32 & 42 & 44 & 52 & 54 & 60 & 64 \\
\hline 14 & 72 & 64 & 57 & 50 & & 32 & 41 & 45 & 52 & 55 & 59 & 64 \\
\hline 15 & 70 & 63 & 57 & 50 & & 32 & 41 & -- & 52 & 54 & 59 & 64 \\
\hline 16 & 70 & 63 & 57 & 50 & & 32 & 41 & 45 & 52 & 54 & 58 & 64 \\
\hline 17 & 72 & 63 & 57 & 50 & & 32 & 40 & 44 & 51 & 54 & 58 & 64 \\
\hline 18 & 74 & 62 & 56 & 50 & & 32 & 40 & 44 & 51 & 55 & 58 & 65 \\
\hline 19 & 74 & 62 & 56 & 48 & & 33 & 40 & 44 & 52 & 56 & 58 & 66 \\
\hline 20 & 74 & 62 & 56 & 48 & & 34 & 40 & 44 & 52 & 56 & 59 & 66 \\
\hline 21 & 73 & $\frac{02}{62}$ & 56 & 46 & & 34 & 40 & 44 & 52 & -- & 60 & 68 \\
\hline 22 & 72 & 62 & 56 & 46 & & 34 & 39 & 44 & 52 & 57 & 60 & 68 \\
\hline 23 & 72 & 62 & 56 & 46 & & 33 & 39 & 44 & 52 & 58 & 60 & 69 \\
\hline 24 & 72 & 62 & 56 & 44 & & 34 & 38 & 44 & 53 & 58 & 60 & 69 \\
\hline 25 & 70 & 62 & 56 & 46 & & 36 & 39 & 44 & 52 & 58 & 60 & 69 \\
\hline 26 & 70 & 62 & 56 & 44 & & 36 & 38 & 45 & 52 & 58 & 60 & 69 \\
\hline 27 & 69 & 63 & 55 & 44 & & 36 & 40 & 45 & 51 & 57 & 60 & -- \\
\hline 28 & 68 & 63 & 56 & 44 & & 36 & 40 & 46 & 50 & 56 & 60 & 70 \\
\hline 29 & 68 & 63 & 54 & 44 & & 37 & -- & 46 & 50 & 56 & 60 & 69 \\
\hline 30 & 68 & 62 & 54 & 43 & & 37 & ----- & 46 & 50 & 56 & 63 & 69 \\
\hline 31 & 68 & ---- & 54 & $-\infty-\infty$ & & 37 & ----- & 46 & ----- & 56 & ----- & 69 \\
\hline Avg & 71 & 64 & 57 & 49 & & 34 & 40 & 43 & 51 & 55 & 59 & 66 \\
\hline Max & 74 & 67 & 62 & 54 & & 38 & 42 & 46 & 53 & 58 & 63 & 70 \\
\hline Min & 68 & 62 & 54 & 43 & & 32 & 38 & 40 & 47 & 49 & 58 & 64 \\
\hline
\end{tabular}


Table 2.--Daily water temperatures of Columbia River at Prindle, Wash., August 1941 to July 1942

[Once-daily observations--mixed morning and afternoon readings at river mile 134.5]

\begin{tabular}{|c|c|c|c|c|c|c|c|c|c|c|c|c|}
\hline Day & August & September & October & November & December & January & February & March & April & May & June & July \\
\hline 1 & & 66 & 62 & 54 & 46 & 36 & 39 & 41 & 48 & 50 & 57 & 64 \\
\hline 2 & & 66 & 61 & 52 & -- & -- & -- & 40 & -- & 51 & 57 & 66 \\
\hline 3 & & 66 & 60 & 54 & 45 & 34 & 38 & 42 & 50 & -- & 58 & 66 \\
\hline 4 & & 66 & 60 & 53 & 46 & & 38 & 40 & 50 & 50 & 59 & 66 \\
\hline 5 & & 66 & 59 & 54 & 46 & & -- & 42 & 50 & -- & - & 66 \\
\hline 6 & & 65 & 58 & 55 & 46 & & 40 & 41 & 50 & 54 & 59 & 65 \\
\hline 7 & & 65 & 58 & -- & 46 & 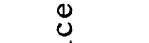 & 40 & 44 & 51 & 52 & 60 & -- \\
\hline 8 & & 66 & 58 & 52 & 45 & $\cdot-1$ & 40 & 44 & 52 & 54 & 60 & 66 \\
\hline 9 & & 64 & -- & 53 & 44 & ت्] & 41 & 43 & 52 & 54 & 59 & 65 \\
\hline 10 & & -- & 58 & 52 & 44 & $\pi$ & 41 & 44 & -- & 54 & 58 & 65 \\
\hline 11 & 70 & 65 & 57 & 52 & 44 & 3 & 41 & 44 & 52 & 54 & 58 & 65 \\
\hline 12 & 70 & 64 & 58 & 52 & 44 & E & 42 & 44 & 52 & 55 & 60 & -- \\
\hline 13 & 69 & 64 & 58 & -- & -- & 4 & 42 & 44 & 52 & 55 & 60 & 65 \\
\hline 14 & 72 & 63 & 56 & 50 & -- & 0 & 42 & -- & 52 & 56 & 60 & 65 \\
\hline 15 & 69 & 62 & 58 & 50 & -- & $\ddot{n}$ & 41 & 45 & 53 & 55 & 58 & 64 \\
\hline 16 & 71 & 62 & -- & 50 & 44 & $\vec{\pi}$ & 41 & 45 & 52 & 55 & 59 & 64 \\
\hline 17 & 70 & 62 & 58 & 50 & 43 & U & -- & 44 & 52 & -- & 58 & 64 \\
\hline 18 & 72 & 61 & 57 & 49 & 41 & م & -- & 44 & 53 & 56 & -- & 63 \\
\hline 19 & 74 & 60 & 56 & 50 & 44 & $\stackrel{0}{\oplus}$ & 40 & 44 & 52 & 56 & 59 & 65 \\
\hline 20 & 72 & 61 & 56 & -- & 44 & ?.-1 & 40 & 46 & 54 & 58 & 60 & 65 \\
\hline 21 & 72 & 62 & 57 & 47 & 44 & n & 40 & 45 & 53 & -- & 60 & 68 \\
\hline 22 & 70 & 63 & 57 & 45 & -- & O & 40 & 45 & 52 & 57 & 61 & 68 \\
\hline 23 & 72 & 61 & 56 & 45 & 44 & U్త & 39 & 44 & 53 & 56 & 60 & 69 \\
\hline 24 & 70 & 62 & -- & 46 & 44 & E. & -- & -- & 53 & 58 & 60 & 70 \\
\hline 25 & 70 & 64 & 56 & 46 & -- & $\varepsilon$ & 39 & 44 & 52 & 58 & 60 & 70 \\
\hline 26 & 70 & -- & 56 & 46 & -- & .0 & 40 & -- & 52 & 57 & -- & -- \\
\hline 27 & 68 & 62 & 56 & 44 & -- & 証 & 38 & 47 & -- & 57 & 59 & 68 \\
\hline 28 & 69 & 63 & -- & 41 & -- & 岕 & 41 & 47 & 50 & 58 & 60 & 68 \\
\hline 29 & 68 & 64 & 54 & -- & -- & & -- & 46 & 50 & 56 & 62 & 68 \\
\hline 30 & 68 & 62 & 54 & 46 & 39 & & $---\infty$ & 48 & -- & 56 & 63 & 68 \\
\hline 31 & 67 & $---n-n$ & 53 &.------ & -- & & ------ & -- & $-\cdots-\cdots$ & 56 & $\ldots-\ldots$ & 68 \\
\hline$\overline{\text { Avg }}$ & 70 & 63 & 57 & 49 & 44 & & 40 & 44 & 52 & 55 & 59 & 66 \\
\hline $\operatorname{Max}$ & 74 & 66 & 62 & 55 & 46 & & 42 & 48 & 54 & 58 & 63 & 70 \\
\hline Min & 67 & 60 & 53 & 41 & 39 & & 38 & 40 & 48 & 50 & 57 & 63 \\
\hline
\end{tabular}


Table 3.--Daily water temperatures of Columbia River at Washougal, Wash., August 1941 to July 1942

[Once-daily observations--8 to 10 a.m. at river mile 12l.6]

\begin{tabular}{|c|c|c|c|c|c|c|c|c|c|c|c|c|}
\hline Day & August & September & October & November & December & January & February & March & April & May & June & July \\
\hline 1 & & 66 & 62 & 52 & 46 & 33 & 39 & 39 & 48 & 49 & 56 & 63 \\
\hline 2 & & 66 & 61 & 52 & 48 & 33 & 39 & 44 & 47 & 49 & 57 & 64 \\
\hline 3 & & 65 & 61 & 53 & 45 & 34 & 39 & 40 & 48 & 50 & 57 & 65 \\
\hline 4 & & 66 & 60 & 52 & 45 & 32 & 39 & 40 & 49 & 51 & 57 & 65 \\
\hline 5 & & 66 & 59 & 54 & 46 & 31 & 39 & 44 & 49 & 50 & 58 & 66 \\
\hline 6 & & 65 & 59 & 53 & 46 & 31 & 39 & 42 & 50 & 52 & 59 & 66 \\
\hline 7 & & 64 & 58 & 51 & 46 & 31 & 39 & 38 & 50 & 52 & 59 & 65 \\
\hline 8 & & 65 & 57 & 51 & 45 & 31 & 39 & 43 & 50 & 53 & 60 & 65 \\
\hline 9 & & 65 & 58 & 51 & 43 & -- & 39 & 46 & 50 & 54 & 60 & 66 \\
\hline 10 & & 64 & 57 & 51 & 42 & -- & 39 & 44 & 51 & 53 & 59 & 66 \\
\hline 11 & 70 & 63 & 57 & 51 & 43 & -- & 41 & 45 & 52 & 53 & 58 & 65 \\
\hline 12 & 70 & 64 & 56 & 52 & 42 & -- & 40 & 42 & 51 & 54 & 57 & 64 \\
\hline 13 & 71 & 64 & 56 & 52 & 42 & 31 & 38 & 44 & 52 & 53 & 58 & 64 \\
\hline 14 & 70 & 64 & 55 & 51 & 42 & 32 & 40 & 43 & 51 & 53 & 59 & 64 \\
\hline 15 & 70 & 62 & 55 & 50 & 43 & 32 & 40 & 43 & 52 & 55 & 59 & 64 \\
\hline 16 & 69 & 63 & 55 & 49 & 43 & 32 & 40 & 44 & 52 & 55 & 59 & 64 \\
\hline 17 & 70 & 63 & 56 & 48 & 43 & 32 & 40 & 46 & 50 & 54 & 59 & 64 \\
\hline 18 & 71 & 62 & 56 & 48 & 43 & 32 & 40 & 44 & 52 & 55 & 58 & 63 \\
\hline 19 & 73 & 60 & 56 & 48 & 44 & 32 & 40 & 44 & 51 & 56 & 58 & 65 \\
\hline 20 & 73 & 60 & 55 & 46 & 44 & 32 & 39 & 43 & 52 & 56 & 58 & 65 \\
\hline 21 & 72 & 60 & 55 & 44 & 44 & 32 & 39 & 45 & 52 & 58 & 57 & 66 \\
\hline 22 & 72 & 61 & 54 & 44 & $4 \cdot \hat{r}$ & 32 & 39 & 45 & 51 & 58 & $5 \overline{9}$ & 67 \\
\hline 23 & 72 & 62 & 55 & 44 & 44 & 32 & 39 & 45 & 53 & 57 & 59 & 67 \\
\hline 24 & 70 & 62 & 55 & 42 & 43 & 33 & 39 & 44 & 53 & 57 & 60 & 68 \\
\hline 25 & 70 & 62 & 56 & 46 & 43 & 37 & 40 & 44 & 52 & 58 & 60 & 68 \\
\hline 26 & 69 & 62 & 55 & 44 & 41 & 38 & 39 & 44 & 51 & 57 & 60 & 67 \\
\hline 27 & 68 & 61 & 55 & 44 & 41 & 35 & 39 & 45 & 50 & 57 & 59 & 69 \\
\hline 28 & 68 & 62 & 55 & 44 & 40 & 37 & 39 & 45 & 50 & 57 & 59 & 69 \\
\hline 29 & 67 & 62 & 53 & 44 & 39 & 36 & -- & 46 & 49 & 56 & 60 & 69 \\
\hline 30 & 69 & 62 & 53 & 45 & 38 & 36 & $---\cdots$ & 46 & 50 & 56 & 62 & 68 \\
\hline 31 & 67 & $---\cdots-$ & 52 & $--\cdots--$ & 37 & 36 & $-----n$ & 48 & $-\cdots--$ & 55 & $-\cdots--$ & 69 \\
\hline $\overrightarrow{\mathrm{Avg}}$ & 70 & 63 & 56 & 47 & 43 & 33 & 39 & 44 & 51 & 54 & 59 & 66 \\
\hline Max & 73 & 66 & 62 & 54 & 48 & 38 & 41 & 48 & 53 & 58 & 62 & 69 \\
\hline Min & 67 & 60 & 52 & 42 & 37 & 31 & 38 & 38 & 47 & 49 & 56 & 63 \\
\hline
\end{tabular}


Table 4.--Daily water temperatures of Columbia River at Ellsworth, Wash., August 1941 to July 1942

[once-daily observations-mixed morning and afternoon readings at river mile 1l2.3]

\begin{tabular}{|c|c|c|c|c|c|c|c|c|c|c|c|c|}
\hline Day & August & September & October & November & December & January & February & March & April & May & June & July \\
\hline 1 & & $\overline{-}$ & 62 & 53 & 46 & 37 & 40 & -- & 49 & 50 & 56 & 63 \\
\hline 2 & & 65 & -- & - & 46 & 36 & 41 & 41 & 48 & 50 & -- & 64 \\
\hline 3 & & 65 & 60 & 53 & 46 & 36 & 40 & 41 & 49 & 50 & 57 & -- \\
\hline 4 & & 65 & - & - & 46 & 37 & 40 & 41 & 50 & 51 & -- & 66 \\
\hline 5 & & 65 & -- & 53 & 46 & 32 & 40 & 41 & -- & 51. & 58 & - \\
\hline 6 & & 65 & -- & 53 & 48 & 32 & 40 & -- & 51 & 52 & 59 & 66 \\
\hline 7 & & -- & 58 & 52 & -- & 32 & -- & 41 & 51 & 53 & -- & 65 \\
\hline 8 & & 65 & 58 & 52 & -- & 32 & -- & -- & 51 & 54 & 60 & 65 \\
\hline 9 & & 65 & 58 & -- & 46 & 32 & 41 & 44 & 51 & 54 & 60 & 66 \\
\hline 10 & & -- & 58 & 52 & -- & 32 & 42 & -- & 52 & -- & 59 & 66 \\
\hline 11 & & -- & -- & -- & 44 & -- & 42 & 44 & 52 & 54 & 59 & 65 \\
\hline 12 & 70 & 64 & -- & 52 & -- & 32 & 42 & 44 & -- & 54 & 59 & 65 \\
\hline 13 & 70 & 64 & 58 & 52 & 43 & 33 & 42 & -- & 52 & -- & -- & 65 \\
\hline 14 & 70 & -- & -- & 51 & -- & 33 & 42 & 43 & 52 & 55 & -- & 64 \\
\hline 15 & 71 & 63 & -- & 51 & 44 & 33 & -- & -- & 52 & 55 & 59 & 64 \\
\hline 16 & 70 & 63 & -- & -- & 44 & 33 & 42 & 46 & 52 & 55 & -- & -- \\
\hline 17 & -- & 63 & -- & 50 & 44 & -- & 42 & -- & 52 & -- & 59 & 64 \\
\hline 18 & 71 & -- & - & 49 & -- & -- & 40 & -- & 52 & 55 & 58 & -- \\
\hline 19 & 72 & -- & -- & -- & 47 & -- & 39 & -- & -- & 56 & 57 & -- \\
\hline 20 & 73 & -- & 55 & $=-$ & -- & 36 & 40 & 44 & 52 & -- & -- & 65 \\
\hline 21 & 72 & -- & 55 & 47 & -- & 36 & -- & 43 & 53 & -- & -- & 66 \\
\hline 22 & 72 & 62 & 57 & 46 & 44 & 35 & -- & -- & 52 & 56 & 58 & 67 \\
\hline 23 & 72 & -- & 56 & -- & 44 & 35 & 40 & 45 & 53 & -- & 59 & 67 \\
\hline 24 & -- & -- & -- & 46 & 44 & 35 & 40 & 45 & 53 & 58 & 60 & 68 \\
\hline 25 & 70 & -- & - & 46 & -- & -- & 40 & 44 & 53 & 58 & 60 & 68 \\
\hline 26 & 69 & 62 & -- & 45 & 43 & 36 & 40 & 45 & -- & 58 & 60 & -- \\
\hline 27 & 69 & -- & 55 & 45 & 41 & 38 & 40 & 46 & -- & 57 & 59 & 68 \\
\hline 28 & 68 & -- & 55 & 44 & -- & 38 & -- & 46 & 52 & 58 & 59 & 69 \\
\hline 29 & 68 & -- & 55 & 45 & 40 & 38 & -- & -- & 51 & -- & -- & 69 \\
\hline 30 & -- & 62 & -- & -- & 40 & 38 & ----- & 48 & 50 & 58 & 60 & 69 \\
\hline 31 & -- & ------ & 53 & $---\cdots$ & 39 & 38 & ------ & -- & ------ & -- & ------ & 69 \\
\hline Avg & 70 & 64 & 57 & 49 & 44 & 35 & 41 & 44 & 51 & 54 & 59 & 66 \\
\hline Ma: & 73 & 55 & $6 ?$ & 53 & 48 & 38 & $4 ?$ & 48 & 53 & 58 & 60 & 69 \\
\hline Min & 68 & 62 & 53 & 44 & 39 & 32 & 39 & 41 & 48 & 50 & 56 & 63 \\
\hline
\end{tabular}


Table 5.--Daily water temperatures of Columbia River at Vancouver, Wash., August 1941 to July 1942

[Once-daily observations--mostly afternoon readings at river mile 106.5]

\begin{tabular}{|c|c|c|c|c|c|c|c|c|c|c|c|c|}
\hline$\overline{\text { Day }}$ & August & September & Oetober & November & December & January & February & Mareh & April & May & June & July \\
\hline 1 & & -- & 62 & $-\infty$ & -- & -- & 38 & 40 & 48 & 50 & 58 & -- \\
\hline 2 & & 67 & -- & 54 & -- & 37 & -- & -- & 49 & -- & 58 & -- \\
\hline 3 & & -- & -- & 54 & 46 & $-\infty$ & 38 & 41 & 49 & $-\infty$ & -- & -- \\
\hline 4 & & 66 & 61 & 54 & 46 & 36 & -- & -- & -- & 51 & 58 & -- \\
\hline 5 & & -- & 60 & -- & 46 & -- & 39 & 41 & 50 & -- & -- & 67 \\
\hline 6 & & -- & 60 & 54 & -- & 32 & -- & 41 & -- & -- & 59 & -- \\
\hline 7 & & -- & - & -- & -- & -- & 41 & 42 & 51 & 54 & -- & -- \\
\hline 8 & & 66 & -- & -- & 46 & - & - & -- & 51 & 54 & 58 & -- \\
\hline 9 & & 66 & -- & 52 & 46 & -- & 42 & 42 & -- & 54 & -- & 67 \\
\hline 10 & & 65 & 58 & 52 & -- & 33 & -- & -- & 52 & -- & 58 & -- \\
\hline 11 & & -- & 58 & 52 & 44 & 33 & -- & 45 & 52 & 54 & 58 & -- \\
\hline 12 & 71 & 65 & -- & 52 & -- & 33 & -- & 44 & -- & 55 & 58 & 66 \\
\hline 13 & 72 & -- & 57 & -- & -- & 33 & 42 & -- & 53 & 56 & 58 & -- \\
\hline 14 & 72 & -- & 58 & -- & -- & 33 & -- & -- & 54 & 56 & -- & 65 \\
\hline 15 & -- & 63 & - & -- & 44 & 33 & 42 & -- & -- & -- & -- & 65 \\
\hline 16 & -- & 64 & -- & 50 & 44 & 34 & -- & 46 & 53 & -- & 58 & 65 \\
\hline 17 & -- & -- & 58 & -- & 44 & 34 & -- & 44 & 52 & 56 & -- & 65 \\
\hline 18 & 73 & - & -- & 50 & 44 & 34 & 41 & -- & 52 & - & 58 & - \\
\hline 19 & 74 & -- & -- & 50 & -- & - & 41 & 45 & -- & -- & -- & -- \\
\hline 20 & 74 & 63 & 58 & -- & -- & 36 & 40 & - & 52 & 55 & -- & 67 \\
\hline 21 & 74 & -- & 58 & -- & 44 & 36 & 40 & 45 & -- & -- & 59 & 67 \\
\hline 22 & 74 & 63 & 57 & -- & 44 & -- & -- & -- & 53 & 56 & -- & \\
\hline 23 & -- & 63 & 57 & 46 & -- & 36 & 40 & -- & -- & -- & 59 & \\
\hline 24 & 72 & -- & 57 & 46 & 45 & 36 & 40 & 45 & 53 & 58 & 59 & \\
\hline 25 & -- & 64 & -- & -- & -- & -- & 40 & 45 & 53 & 58 & 59 & \\
\hline 26 & $\cdots$ & 63 & -- & -- & 42 & 37 & 40 & 45 & -- & -- & -- & \\
\hline 27 & 69 & -- & 56 & -- & 41 & -- & -- & 46 & 52 & 58 & 59 & \\
\hline 28 & 69 & 63 & -- & -- & 42 & 39 & 40 & -- & 52 & 58 & -- & \\
\hline 29 & -- & -- & - & -- & 42 & 39 & -- & -- & -- & 58 & 60 & \\
\hline 30 & - & 63 & -- & -- & 40 & 38 & ----- & 46 & 50 & 58 & 60 & \\
\hline 31 & 68 & ------ & -- & $-\ldots--$ & 39 & 38 & $-m---$ & 48 & ----- & 58 & $-----\infty$ & \\
\hline$\overline{\text { Avg }}$ & 72 & 64 & 58 & 51 & 44 & 35 & 40 & 44 & 51 & 56 & 59 & 66 \\
\hline Max & 74 & 67 & 62 & 54 & 46 & 39 & 42 & 48 & 54 & 58 & 60 & 67 \\
\hline Min & 68 & 63 & 56 & 46 & 39 & 32 & 38 & 40 & 48 & 50 & 58 & 65 \\
\hline
\end{tabular}


Table 6.--Daily water temperatures of Columbia River at Kelley Point, Oreg., August 1941 to July 1942

[Once-daily observations--mixed morning and afternoon readings at river mile lol.8]

\begin{tabular}{|c|c|c|c|c|c|c|c|c|c|c|c|c|}
\hline Day & August & September & October & November & December & January & February & March & April & May & June & July \\
\hline 1 & & 66 & 62 & 54 & 47 & 36 & -- & -- & 50 & 50 & 59 & 72 \\
\hline 2 & & 68 & 62 & -- & 48 & 34 & 45 & 47 & 52 & 54 & 60 & 71 \\
\hline 3 & & 68 & 62 & 55 & 48 & 39 & 47 & 47 & 54 & -- & 60 & 72 \\
\hline 4 & & 66 & 60 & 54 & 48 & $-\infty$ & 47 & 46 & 53 & 54 & 61 & 71 \\
\hline 5 & & 66 & - & 55 & 47 & 34 & 46 & 49 & -- & 57 & 63 & -- \\
\hline 6 & & 65 & 59 & 56 & 46 & 35 & 44 & 50 & 53 & 60 & 62 & 68 \\
\hline 7 & & -- & 60 & 56 & -- & 34 & 46 & 47 & 54 & 58 & $-\infty$ & 68 \\
\hline 8 & & 66 & 60 & 55 & 45 & 36 & -- & -- & 51 & 56 & 60 & 69 \\
\hline 9 & & 66 & 59 & - & 44 & 36 & 46 & 49 & 55 & 56 & 60 & 70 \\
\hline 10 & & 64 & 61 & 55 & 45 & 36 & 46 & 49 & 54 & -- & 60 & 69 \\
\hline 11 & 70 & 63 & 59 & 54 & 44 & -- & 47 & 48 & 56 & 56 & 60 & 68 \\
\hline 12 & 70 & 64 & -- & 54 & 43 & 38 & 46 & 48 & -- & 55 & 61 & $-\infty$ \\
\hline 13 & 70 & 63 & 57 & 54 & 44 & 40 & 46 & 48 & 53 & 54 & 62 & 68 \\
\hline 14 & 70 & -- & 55 & 52 & - & 38 & 45 & 44 & 58 & 57 & -- & 68 \\
\hline 15 & 70 & 64 & 58 & 53 & 46 & 39 & -- & -- & 54 & 58 & 60 & 66 \\
\hline 16 & 70 & 64 & 57 & -- & 46 & 39 & 45 & 50 & 54 & 54 & 60 & 66 \\
\hline 17 & -- & 64 & 58 & 50 & 48 & 40 & 45 & 48 & 54 & -- & 60 & 66 \\
\hline 18 & 71 & 62 & 56 & 48 & 49 & -- & 46 & 49 & 56 & 58 & 60 & 67 \\
\hline 19 & 72 & 62 & -- & 48 & 48 & 38 & 46 & 48 & -- & 56 & 60 & -- \\
\hline 20 & 73 & 62 & 58 & 48 & 46 & 43 & 44 & 50 & 58 & 59 & 61 & 69 \\
\hline 21 & 71 & -- & 59 & 45 & -- & 44 & 44 & 51 & 57 & 58 & -- & 71 \\
\hline 22 & 73 & 64 & 58 & 45 & 48 & 42 & - & -- & 57 & 58 & 62 & 71 \\
\hline 23 & 72 & 64 & 58 & - & 46 & 40 & 44 & 49 & 55 & 58 & 62 & 71 \\
\hline 24 & 71 & 62 & 58 & 46 & 46 & 40 & 44 & 48 & 57 & -- & 62 & 71 \\
\hline 25 & -- & 62 & 58 & 47 & -- & -- & 45 & 48 & 54 & 60 & 62 & 70 \\
\hline 26 & 69 & 62 & -- & 45 & 42 & 44 & $\overline{44}$ & 49 & -- & 60 & 62 & -- \\
\hline 27 & 69 & 62 & 58 & 46 & 41 & 44 & 44 & 47 & 52 & 59 & 62 & 71 \\
\hline 28 & 68 & -- & 56 & 46 & -- & 43 & 46 & 49 & 53 & 58 & -- & 69 \\
\hline 29 & 68 & 62 & 53 & 47 & 42 & 43 & -- & -- & 54 & 58 & 66 & 70 \\
\hline 30 & 68 & 62 & 55 & -- & 39 & 45 & ------ & 54 & 52 & 58 & 67 & 70 \\
\hline 31 & -- & $---n$ & 54 & $---\cdots$ & 38 & -- & ------ & 54 & $---\cdots$ & -- & ------ & -- \\
\hline$\overline{\text { Avg }}$ & 70 & 64 & 58 & 51 & 45 & 39 & 45 & 49 & 54 & 57 & 61 & 69 \\
\hline $\operatorname{Max}$ & 73 & 68 & 62 & 56 & 49 & 45 & 47 & 54 & 58 & 60 & 67 & 72 \\
\hline Min & 68 & 62 & 53 & 45 & 38 & 34 & 44 & 44 & 50 & 50 & 59 & 66 \\
\hline
\end{tabular}


Table 7.--Daily water temperatures of Willamette River at Portland, Oreg. (Morrison Bridge), August 1941 to July 1942

[Once-daily observations--mixed morning and afternoon readings at river mile 12.8 ]

\begin{tabular}{|c|c|c|c|c|c|c|c|c|c|c|c|c|}
\hline Day & August & September & O-tober & November & December & January & February & March & April & May & June & July \\
\hline 1 & & 71 & 62 & 54 & 44 & 38 & -- & -- & 51 & 52 & 57 & 65 \\
\hline 2 & & 70 & 62 & - & 46 & 36 & 44 & 45 & 52 & 52 & -- & 66 \\
\hline 3 & & 69 & 62 & 54 & 48 & 37 & 45 & 45 & 53 & 51 & 58 & 70 \\
\hline 4 & & 68 & 62 & 53 & 49 & -- & 46 & 46 & -- & 51 & 60 & 72 \\
\hline 5 & & 68 & -- & 54 & 48 & 34 & 46 & 47 & -- & $=$ & - & 73 \\
\hline 6 & & 68 & 60 & 54 & 48 & 34 & 46 & 46 & 53 & 52 & 60 & 74 \\
\hline 7 & & -- & 60 & 54 & -- & 34 & 45 & 48 & 53 & 54 & 60 & -- \\
\hline 8 & & 65 & 60 & 54 & 47 & 35 & -- & -- & 52 & 56 & 61 & 75 \\
\hline 9 & & 65 & 58 & -- & 46 & 35 & 45 & 47 & 55 & 56 & 61 & 75 \\
\hline 10 & & 68 & 59 & 53 & 45 & 38 & 46 & 47 & 55 & -- & - & 75 \\
\hline 11 & & 66 & 58 & 53 & 44 & -- & 46 & 48 & 55 & 56 & 60 & 73 \\
\hline 12 & 77 & 66 & -- & 53 & 44 & 39 & 47 & 48 & -- & -- & 59 & 71 \\
\hline 13 & 74 & 65 & 56 & 53 & 44 & 40 & 46 & 47 & 55 & 55 & 60 & 72 \\
\hline 14 & 74 & -- & 56 & 53 & -- & 40 & 45 & 47 & 55 & 56 & 60 & 72 \\
\hline 15 & 75 & 64 & 56 & 52 & 44 & 40 & -- & -- & 56 & 56 & 60 & 72 \\
\hline 16 & 75 & 64 & 58 & -- & 45 & 40 & 43 & 46 & 56 & 56 & -- & 70 \\
\hline 17 & -- & 64 & 59 & 48 & 46 & 40 & 43 & 45 & 55 & 56 & 62 & 69 \\
\hline 18 & 76 & 63 & 56 & 48 & 47 & -- & 43 & 46 & 54 & 57 & 62 & 69 \\
\hline 19 & 76 & 62 & -- & 48 & 47 & 40 & 44 & 46 & -- & -- & 61 & 69 \\
\hline 20 & 76 & 63 & 54 & 46 & 48 & 41 & 44 & 48 & 55 & 58 & 61 & 70 \\
\hline 21 & 75 & -- & 55 & 46 & -- & 40 & 44 & 48 & 55 & 59 & 60 & -- \\
\hline 22 & 74 & 62 & 57 & 45 & 47 & 40 & -- & -- & 55 & 60 & 61 & 71 \\
\hline 23 & 76 & 61 & 56 & -- & 46 & 40 & 44 & 47 & 55 & 60 & - & 71 \\
\hline 24 & -- & 63 & 57 & 44 & 46 & 41 & 43 & 47 & 56 & 60 & 62 & 72 \\
\hline 25 & 74 & 62 & 56 & 45 & -- & -- & 43 & 47 & 55 & 61 & 62 & 72 \\
\hline 26 & 73 & 63 & -- & 45 & 44 & 43 & 44 & 48 & 55 & -- & 63 & 73 \\
\hline 27 & 74 & 64 & 55 & 44 & 42 & 44 & 44 & 48 & 54 & 58 & 63 & 74 \\
\hline 28 & 72 & -- & 55 & 45 & -- & 46 & 44 & 48 & -- & 57 & 63 & -- \\
\hline 29 & 71 & 62 & 56 & 46 & 41 & 46 & -- & -- & 52 & 56 & 64 & 74 \\
\hline 30 & 72 & 62 & 55 & -- & 40 & 45 & $---n$ & 49 & -- & 56 & -- & 74 \\
\hline 31 & -- & $--\infty-\infty$ & 54 & ----- & 40 & 46 & $-\cdots---$ & 50 & $---\cdots$ & 56 & ------ & 74 \\
\hline$\overline{\text { Avg }}$ & 74 & 65 & 57 & 50 & 45 & 40 & 45 & 47 & 54 & 56 & 61 & 72 \\
\hline Max & 77 & 71 & 62 & 54 & 49 & 46 & 47 & 50 & 56 & 61 & 64 & 75 \\
\hline Min & 71 & 61 & 54 & 44 & 40 & 34 & 43 & 45 & 51 & 51 & 57 & 65 \\
\hline
\end{tabular}




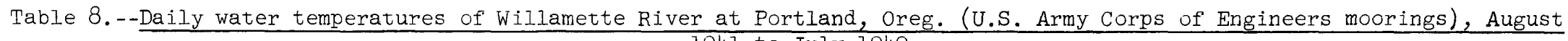
1941 to July 1942

[Once-daily observations--morning readings to Feb. 23, afternoon readings thereafter, at river mile 6.3]

\begin{tabular}{|c|c|c|c|c|c|c|c|c|c|c|c|c|}
\hline Day & August & September & October & November & December & January & February & March & April & May & June & July \\
\hline 1 & & -- & 61 & 54 & 45 & 35 & -- & -- & 51 & 54 & 58 & 69 \\
\hline 2 & & 70 & 62 & -- & 46 & 34 & 44 & 46 & 53 & 54 & 60 & 70 \\
\hline 3 & & 71 & 62 & 54 & 46 & 33 & 44 & 47 & 54 & -- & 60 & 73 \\
\hline 4 & & 70 & 62 & 54 & 48 & -- & 46 & 46 & 53 & 52 & 63 & 72 \\
\hline 5 & & 70 & -- & 54 & 48 & 32 & 46 & 48 & -- & 56. & 63 & -- \\
\hline 6 & & 66 & 62 & 54 & 48 & 32 & 46 & 48 & 54 & 56 & 60 & 73 \\
\hline 7 & & -- & 62 & 54 & -- & 32 & 45 & 48 & 54 & 57 & -- & 76 \\
\hline 8 & 78 & 66 & 60 & 54 & 48 & 32 & -- & -- & 54 & 55 & 61 & 76 \\
\hline 9 & 76 & 66 & 60 & -- & 47 & 32 & 46 & 48 & 54 & 56 & 62 & 76 \\
\hline 10 & -- & 66 & 60 & 53 & 46 & 35 & 46 & 48 & 58 & -- & 63 & 75 \\
\hline 11 & 74 & 66 & 60 & -- & 46 & -- & 46 & 48 & 55 & 57 & 62 & 74 \\
\hline 12 & 73 & 65 & -- & 53 & 44 & 38 & 46 & 48 & -- & 58 & 64 & -- \\
\hline 13 & 74 & 65 & 58 & 53 & 44 & 40 & 46 & 48 & 56 & 59 & 66 & 72 \\
\hline 14 & 74 & -- & 57 & 53 & $-\infty$ & 40 & 46 & 48 & 56 & 58 & -- & 72 \\
\hline 15 & 73 & 65 & 57 & 52 & 44 & 40 & -- & - & 57 & 58 & 64 & 72 \\
\hline 16 & 73 & 65 & 56 & -- & 45 & 40 & 44 & 48 & 56 & 57 & 61 & 71 \\
\hline 17 & -- & 64 & 57 & 50 & 45 & 40 & 44 & 48 & 57 & -- & 62 & 72 \\
\hline 18 & 74 & 64 & 56 & 49 & 47 & -- & 42 & 47 & 57 & 58 & 64 & 72 \\
\hline 19 & 75 & 63 & $\ldots$ & 46 & 47 & 39 & 42 & 48 & -- & 60 & 65 & -- \\
\hline 20 & 74 & 61 & 56 & -- & 47 & 40 & -- & 49 & 58 & 60 & 66 & 76 \\
\hline 21 & 73 & -- & 56 & 46 & -- & 40 & 44 & 48 & 56 & 60 & -- & 76 \\
\hline 22 & 73 & 60 & 56 & 45 & 47 & 40 & -- & -- & 55 & 60 & 66 & 73 \\
\hline 23 & 74 & 61 & 56 & - & 46 & 40 & 44 & 48 & 56 & 60 & 66 & 75 \\
\hline 24 & -- & 62 & 56 & 44 & 46 & 40 & 44 & 48 & $\ldots$ & -- & 66 & 74 \\
\hline 25 & 73 & 62 & 56 & 45 & $=$ & - & 44 & 48 & 56 & 61 & 62 & 76 \\
\hline 26 & 73 & 62 & -- & 44 & 44 & 42 & 44 & 48 & -- & 62 & 64 & -- \\
\hline 27 & 72 & 60 & 56 & 44 & 42 & 44 & 44 & 50 & 55 & 60 & 65 & 74 \\
\hline 28 & 72 & -- & 56 & 45 & - & 44 & 46 & 50 & 56 & 59 & - & 74 \\
\hline 29 & 72 & 62 & 55 & 46 & 41 & 44 & -- & -- & 56 & 58 & 72 & 74 \\
\hline 30 & 73 & 62 & 55 & -- & 41 & 45 & $-\cdots--$ & 51 & 54 & 58 & 69 & 74 \\
\hline 31 & -- & ------ & 55 & ------ & 40 & 44 & $-\cdots---$ & 52 & $-\cdots--$ & -- & ------ & 74 \\
\hline$\overline{\text { Avg }}$ & 74 & 65 & 58 & 50 & 45 & 38 & 45 & 48 & 55 & 58 & 64 & 73 \\
\hline Max & 78 & 71 & 62 & 54 & 48 & 45 & 46 & 52 & 58 & 62 & 72 & 76 \\
\hline Min & 72 & 60 & 55 & 44 & 40 & 32 & 42 & 46 & 51 & 52 & 58 & 69 \\
\hline
\end{tabular}


Table 9.--Daily water temperatures of Columbia River at Willow-Bar, Wash., August 1941 to July 1942

[Once-daily observations--mostly mid-day readings at river mile 101.6]

\begin{tabular}{|c|c|c|c|c|c|c|c|c|c|c|c|c|}
\hline Day & August & September & October & November & December & January & February & March & April & May & June & July \\
\hline 1 & & 68 & 62 & 54 & 47 & 36 & 38 & 40 & 48 & 51 & 59 & 66 \\
\hline 2 & & 66 & 62 & 54 & 48 & 35 & 38 & 42 & 50 & 52 & 59 & 68 \\
\hline 3 & & 66 & 62 & 55 & 46 & 35 & 40 & 42 & 49 & 52 & 58 & 68 \\
\hline 4 & & 67 & 60 & 54 & 46 & 34 & 40 & 41 & 52 & 52 & 60 & 68 \\
\hline 5 & & 67 & 60 & 55 & 46 & 33 & 39 & .42 & 52 & 54. & 60 & 68 \\
\hline 6 & & 66 & 60 & -- & 47 & 32 & 40 & 42 & 51 & 56 & 60 & 68 \\
\hline 7 & & 66 & 59 & 53 & 47 & 32 & 40 & 42 & 51 & 56 & 60 & 68 \\
\hline 8 & & 65 & 58 & 52 & 46 & 32 & 40 & 43 & 52 & 56 & 60 & 68 \\
\hline 9 & & 65 & 58 & 53 & 46 & 32 & 41 & 44 & 53 & 55 & 61 & 69 \\
\hline 10 & & 65 & 58 & 58 & 45 & 32 & 42 & 44 & 54 & 56 & 61 & 68 \\
\hline 11 & & 66 & 58 & 54 & 44 & 32 & 42 & 46 & 54 & 56 & 60 & 67 \\
\hline 12 & 70 & 64 & 58 & 53 & 44 & 32 & 41 & 44 & 53 & 58 & 60 & 67 \\
\hline 13 & 70 & 64 & 57 & 53 & 44 & 32 & 41 & 45 & 54 & 57 & 60 & 68 \\
\hline 14 & 70 & 64 & 56 & 52 & 43 & 32 & 41 & 44 & 54 & 57 & 60 & 66 \\
\hline 15 & 70 & 63 & 57 & 52 & 44 & 32 & 41 & 44 & 54 & 58 & 60 & 66 \\
\hline 16 & 70 & 63 & 57 & 50 & 44 & 32 & 42 & 46 & 54 & 56 & 60 & 66 \\
\hline 17 & 70 & 63 & 58 & 50 & 44 & 34 & 42 & 46 & 54 & 57 & 60 & 66 \\
\hline 18 & 71 & 62 & 58 & 50 & 44 & 34 & 42 & 46 & 54 & 58 & 60 & 66 \\
\hline 19 & 72 & 62 & 57 & 50 & 45 & 34 & 42 & 46 & 54 & 58 & 60 & 67 \\
\hline 20 & 72 & 61 & 56 & 49 & 46 & 34 & 40 & 46 & 54 & 59 & 60 & 68 \\
\hline 21 & 73 & 61 & 56 & 48 & 45 & 35 & 41 & 45 & 54 & 60 & 60 & 69 \\
\hline 22 & 73 & 61 & 56 & 47 & 45 & 35 & 40 & 46 & 53 & 60 & 60 & 68 \\
\hline 23 & 73 & 62 & 56 & 46 & 44 & 34 & 40 & 46 & 54 & 60 & 62 & 69 \\
\hline 24 & 72 & 62 & 56 & 46 & 44 & 35 & 40 & 46 & 54 & 60 & 62 & 70 \\
\hline 25 & 72 & 62 & 56 & 46 & 44 & 36 & 40 & 46 & 54 & 60 & 61 & 69 \\
\hline 26 & 70 & 63 & 56 & 46 & 42 & 36 & 40 & 46 & 54 & 60 & 62 & 70 \\
\hline 27 & 70 & 62 & 57 & 46 & 40 & 38 & 40 & 46 & 52 & 58 & 61 & 70 \\
\hline 28 & 68 & 62 & 56 & 45 & 40 & 38 & 40 & 47 & 54 & 59 & 61 & 70 \\
\hline 29 & 68 & 63 & 56 & 45 & 40 & 37 & -- & 47 & 54 & 58 & 62 & 70 \\
\hline 30 & 68 & 63 & 54 & 46 & 40 & 37 & $\ldots$ & 48 & 52 & 58 & 64 & 70 \\
\hline 31 & 68 & $---\cdot-$ & 54 & ------ & 39 & 37 & $-\cdots-\cdots$ & 48 & $-\cdots-\cdots$ & 58 & $\ldots$ & 70 \\
\hline$\overline{\text { Avg }}$ & 70 & 64 & 58 & 50 & 44 & 34 & 40 & 45 & 53 & 57 & 60 & 68 \\
\hline Max & 73 & 68 & 62 & 55 & 48 & 38 & 42 & 48 & 54 & 60 & 64 & 70 \\
\hline Min & 68 & 61 & 54 & 45 & 39 & 32 & 38 & 40 & 48 & 51 & 58 & 66 \\
\hline
\end{tabular}


Table 10.--Daily water temperatures of Columbia River at St. Helens, Oreg., August 1941 to July 1942

[Once-daily observations--mostly morning readings at river mile 85.8]

$\stackrel{N}{\infty}$

\begin{tabular}{|c|c|c|c|c|c|c|c|c|c|c|c|c|}
\hline Day & August & September & October & November & December & January & February & March & April & May & June & July \\
\hline 1 & & 68 & 62 & 54 & 45 & 37 & 45 & 43 & 47 & 51 & 56 & 65 \\
\hline 2 & & 67 & 61 & -- & 46 & 35 & 43 & 42 & 48 & 50 & 57 & 66 \\
\hline 3 & & 67 & 62 & 54 & 44 & 36 & 42 & 40 & 48 & 51 & 58 & 66 \\
\hline 4 & & 66 & 61 & 53 & 45 & 34 & 41 & 44 & 50 & 51 & 58 & 67 \\
\hline 5 & & 66 & 60 & 55 & 48 & 33 & 43 & 42 & 50 & 51. & 59 & 67 \\
\hline 6 & & 65 & 61 & 53 & 48 & 32 & 44 & 42 & 50 & 52 & 60 & 68 \\
\hline 7 & & 65 & 58 & 53 & 46 & 32 & 44 & 43 & 51 & 53 & 60 & 67 \\
\hline 8 & 71 & 65 & 58 & 52 & 45 & 32 & 45 & 43 & 52 & 54 & 60 & 67 \\
\hline 9 & 69 & 65 & 58 & 52 & 45 & 32 & 45 & 45 & 53 & 54 & 60 & 69 \\
\hline 10 & 70 & 65 & 58 & 52 & 45 & 32 & 45 & 44 & - & 55 & 60 & 69 \\
\hline 11 & 70 & 64 & 58 & 52 & 43 & 32 & 45 & 46 & 54 & 55 & 60 & 68 \\
\hline 12 & 70 & 64 & -- & 52 & 42 & 32 & 44 & 46 & 53 & -- & 60 & 66 \\
\hline 13 & 70 & -- & 58 & 52 & 43 & 35 & 45 & 44 & 53 & -- & 60 & 67 \\
\hline 14 & 69 & 64 & 58 & 52 & 43 & 38 & 45 & 45 & 53 & 53 & 60 & 66 \\
\hline 15 & 71 & 63 & 58 & 52 & 44 & 35 & 44 & 46 & 53 & 56 & 60 & 66 \\
\hline 16 & 70 & 64 & 57 & 51 & 44 & 38 & 42 & 45 & 54 & 55 & 59 & 65 \\
\hline 17 & 71 & 64 & 57 & 50 & 44 & 38 & 42 & 45 & 53 & 56 & 59 & 65 \\
\hline 18 & 70 & 62 & 57 & 48 & 45 & 36 & 41 & 44 & 52 & 56 & 59 & 65 \\
\hline 19 & 72 & 62 & 57 & 48 & 47 & 36 & 40 & 44 & 53 & 57 & 59 & 66 \\
\hline 20 & 71 & 61 & 55 & 46 & 46 & 36 & 41 & 43 & 55 & 57 & 59 & 67 \\
\hline 21 & 72 & 61 & 56 & 46 & 46 & 38 & 41 & 44 & -- & 59 & 60 & 68 \\
\hline 22 & 72 & 61 & 56 & 45 & 47 & 38 & 40 & 44 & 53 & 58 & 60 & 68 \\
\hline 23 & 72 & 62 & 56 & 45 & 45 & 38 & 42 & 44 & 53 & 58 & 60 & 68 \\
\hline 24 & 73 & 62 & 56 & 45 & 45 & 39 & 42 & 46 & 54 & 59 & 61 & 68 \\
\hline 25 & 71 & 62 & 55 & 45 & 44 & 40 & 42 & 45 & 54 & 60 & 60 & 70 \\
\hline 26 & 71 & 62 & 55 & 44 & 40 & 42 & 41 & 45 & 53 & 59 & 60 & 70 \\
\hline 27 & 69 & 61 & 55 & 43 & 42 & 42 & 42 & 45 & 53 & 59 & 60 & 70 \\
\hline 28 & 69 & 61 & 55 & 44 & 41 & 43 & 43 & 47 & 53 & 58 & 61 & 70 \\
\hline 29 & 69 & 62 & 55 & 44 & 40 & 43 & -- & 47 & 51 & 57 & 62 & 69 \\
\hline 30 & 69 & 61 & 54 & 45 & 40 & 44 & $-m---$ & 47 & 52 & 56 & 63 & 68 \\
\hline 31 & 69 & ------ & 54 & $---\cdots--$ & 39 & 44 & $-\cdots-\cdots$ & 48 & $-\cdots--$ & 56 & $-\cdots-\cdots$ & 68 \\
\hline Avg & 70 & 64 & 57 & 49 & 44 & 37 & 43 & 44 & 52 & 55 & 60 & 67 \\
\hline Max & 73 & 68 & 62 & 55 & 48 & 44 & 45 & 48 & 55 & 60 & 63 & 70 \\
\hline Min & 69 & 61 & 54 & 43 & 39 & 32 & 40 & 40 & 47 & 50 & 56 & 65 \\
\hline
\end{tabular}


Taljle 11.--Daily water temperatures of Columbia River at Kalama, Wash., August 1941 to July 1942

[Once-daily observations-mostly morning readings at river mile 75.1]

\begin{tabular}{|c|c|c|c|c|c|c|c|c|c|c|c|c|}
\hline Day & August & September & October & November & December & January & February & March & April & May & June & July \\
\hline 1 & & 67 & 62 & 54 & \begin{tabular}{l|}
45 \\
\end{tabular} & 38 & 38 & 40 & 46 & 50 & 56 & 63 \\
\hline 2 & & 66 & 62 & 54 & 46 & 36 & 39 & 40 & 47 & 49 & 56 & 64 \\
\hline 3 & & 66 & 62 & 54 & 46 & 36 & 40 & 40 & 47 & -- & 57 & 65 \\
\hline 4 & & 66 & 61 & 54 & 46 & 35 & 40 & 40 & 48 & 50 & 57 & 66 \\
\hline 5 & & 66 & 60 & 53 & 46 & 34 & 40 & 40 & 50 & 51 & 58 & 66 \\
\hline 6 & & 66 & 60 & 53 & 46 & 32 & 40 & 40 & 50 & 52 & 58 & 66 \\
\hline 7 & & 65 & 59 & 52 & 46 & 32 & 40 & 41 & 50 & 53 & 59 & 66 \\
\hline 8 & & 65 & 59 & 52 & 46 & 32 & 40 & 42 & 50 & 54 & 59 & 66 \\
\hline 9 & & 64 & 58 & 52 & 46 & 32 & 41 & 43 & 50 & 54 & 59 & 66 \\
\hline 10 & & 64 & 57 & 52 & 45 & 32 & 42 & 43 & 51 & 54 & 59 & 66 \\
\hline 11 & & 64 & 57 & 52 & 44 & 32 & 42 & 43 & 52 & 53 & 58 & 65 \\
\hline 12 & 70 & 64 & 57 & 52 & 44 & 32 & 42 & 43 & 52 & 54 & 58 & 65 \\
\hline 13 & 71 & 64 & 56 & 52 & 43 & 32 & 42 & 43 & 52 & 54 & 58 & 65 \\
\hline 14 & 70 & 64 & 56 & 52 & 43 & 32 & 42 & 43 & 51 & 55 & 58 & 65 \\
\hline 15 & 70 & 64 & 56 & 51 & 44 & 32 & 42 & -- & 52 & 54 & 58 & 65 \\
\hline 16 & 70 & 63 & 56 & 51 & 44 & 35 & 42 & -- & 52 & 55 & 58 & 64 \\
\hline 17 & 70 & 63 & 56 & 50 & 44 & 34 & 41 & 45 & 51 & 55 & -- & 64 \\
\hline 18 & 72 & 63 & 57 & 50 & 44 & 34 & 41 & 45 & 51 & 54 & 58 & 64 \\
\hline 19 & 71 & 62 & 56 & 49 & 44 & 34 & 40 & 44 & 52 & -- & 58 & 64 \\
\hline 20 & 72 & 62 & 56 & 48 & 45 & 34 & 40 & 44 & 52 & 55 & 58 & 65 \\
\hline 21 & 73 & 61 & 56 & 48 & 44 & 35 & 40 & 44 & 52 & 56 & 58 & 66 \\
\hline 22 & 72 & 61 & 56 & 47 & 44 & 35 & 40 & 44 & 52 & 57 & 58 & 66 \\
\hline 23 & 72 & 62 & 56 & 46 & 44 & 35 & 40 & 44 & 52 & 56 & 59 & 66 \\
\hline 24 & 72 & 62 & 56 & 46 & 44 & 35 & 40 & 45 & 52 & 58 & 60 & 67 \\
\hline 25 & 71 & 62 & 56 & 46 & -- & 36 & 39 & 44 & 52 & 58 & 59 & 68 \\
\hline 26 & 70 & 62 & 56 & 46 & 43 & 37 & 40 & 44 & 52 & 58 & 60 & 68 \\
\hline 27 & 70 & 62 & 56 & 44 & 42 & 38 & 40 & 44 & 51 & 56 & 59 & 68 \\
\hline 28 & 69 & 62 & 56 & 45 & 40 & 38 & 40 & 46 & 51 & 56 & 59 & 68 \\
\hline 29 & 68 & 62 & 55 & 44 & 40 & 38 & -- & 46 & 50 & 56 & 59 & 68 \\
\hline 30 & 68 & 62 & 54 & 45 & 40 & 37 & ------ & 46 & 50 & 56 & 61 & 68 \\
\hline 31 & 68 & ------ & 54 & ------ & 38 & 38 & ----- & 47 & ------ & 56 & ------ & 68 \\
\hline $\overrightarrow{\text { Avg }}$ & 70 & 64 & 57 & 50 & 44 & 35 & 40 & 43 & 51 & 54 & 58 & 66 \\
\hline Max & 73 & 67 & 62 & 54 & 46 & 38 & 42 & 47 & 52 & 58 & 61 & 68 \\
\hline Min & 68 & 61 & 54 & 44 & 38 & 32 & 38 & 40 & 46 & 49 & 56 & 63 \\
\hline
\end{tabular}


Table 12.--Daily water temperatures of Columbia River at Longview, Wash., August 1941 to July 1942

[Once-daily observations--mostly between $10 \mathrm{a} . \mathrm{m}$. and $2 \mathrm{p} . \mathrm{m}$. at river mile 66.0]

\begin{tabular}{|c|c|c|c|c|c|c|c|c|c|c|c|c|}
\hline Day & August & September & October & November & December & January & February & March & April & May & June & July \\
\hline 1 & & -- & 62 & 53 & 47 & 37 & 41 & 42 & 49 & 50 & 56 & 65 \\
\hline 2 & & 67 & 62 & -- & 48 & 34 & 43 & 43 & 49 & 49 & 56 & 66 \\
\hline 3 & & 65 & 61 & 53 & 48 & 35 & 43 & 43 & 49 & - & 57 & 66 \\
\hline 4 & & 66 & 61 & 53 & 45 & -- & 43 & 43 & 49 & 50 & 58 & 66 \\
\hline 5 & & 66 & - & 53 & 44 & 34 & 44 & 42 & -- & 52 & 59 & -- \\
\hline 6 & & 66 & 59 & 52 & 45 & 33 & 42 & 43 & 50 & 53 & 60 & 67 \\
\hline 7 & & -- & 57 & 52 & -- & 33 & 42 & 43 & 50 & 55 & -- & 67 \\
\hline 8 & & 65 & 57 & 52 & 45 & 34 & -- & -- & 50 & 55 & 58 & 66 \\
\hline 9 & & 65 & 57 & -- & 44 & 33 & 43 & 44 & 51 & 54 & 58 & 66 \\
\hline 10 & & 65 & 57 & 52 & 44 & 33 & 43 & 44 & 52 & -- & 57 & 66 \\
\hline 11 & & 64 & 57 & 52 & 44 & -- & 43 & 45 & 53 & 52 & 56 & 65 \\
\hline 12 & 71 & 64 & -- & 52 & 44 & 34 & 43 & 44 & -- & 52 & 55 & -- \\
\hline 13 & 71 & 64 & 57 & 52 & 43 & 35 & 42 & 44 & 51 & 53 & 58 & 65 \\
\hline 14 & 71 & -- & 57 & 51 & -- & 38 & 41 & 44 & 51 & 54 & -- & 65 \\
\hline 15 & 71 & 64 & 57 & 50 & 44 & 35 & -- & - & 50 & 55 & 57 & 64 \\
\hline 16 & 71 & 64 & 57 & -- & 45 & 37 & 42 & 43 & 51 & 56 & 56 & 64 \\
\hline 17 & -- & 63 & 56 & 48 & 45 & 38 & 42 & 44 & 50 & -- & 55 & 64 \\
\hline 18 & 72 & 62 & 56 & 47 & 45 & -- & 41 & 45 & 50 & 57 & 56 & 63 \\
\hline 19 & 72 & 61 & -- & 46 & 46 & 38 & 41 & 45 & -- & 56 & 55 & - \\
\hline 20 & 72 & 61 & 55 & 45 & 45 & 38 & 41 & 45 & 53 & 57 & 56 & 66 \\
\hline 21 & 72 & 60 & 55 & 43 & -- & 38 & 41 & 45 & 53 & 58 & -- & 67 \\
\hline 22 & 72 & 60 & 55 & 42 & 45 & 38 & - & -- & 52 & 57 & 58 & 67 \\
\hline 23 & 72 & 60 & 55 & 42 & 45 & 38 & 41 & 45 & 51 & 56 & 59 & 66 \\
\hline 24 & -- & 61 & 55 & -- & 44 & 38 & 41 & 44 & 52 & -- & 60 & 68 \\
\hline 25 & 71 & 62 & 56 & 44 & -- & 39 & 41 & 44 & 52 & 57 & 59 & 69 \\
\hline 26 & 70 & 62 & -- & 46 & 42 & 41 & 41 & 45 & -- & 57 & 58 & -- \\
\hline 27 & 70 & 63 & 56 & 46 & 40 & 40 & 41 & 45 & 50 & 54 & 58 & 69 \\
\hline 28 & 69 & -- & 56 & 45 & -- & 41 & 42 & 46 & 50 & 54 & -- & 69 \\
\hline 29 & 69 & 63 & 55 & 45 & 40 & 41 & -- & -- & 50 & 55 & 60 & 69 \\
\hline 30 & 69 & 62 & 54 & - & 40 & 41 & ----- & 48 & 51 & 55 & 63 & 69 \\
\hline 31 & -- & 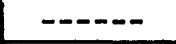 & 54 & $---\cdots$ & 38 & 41 & $---\cdots$ & 49 & $--\cdots--$ & -- & ------- & 68 \\
\hline Avg & 71 & 63 & 57 & 49 & 44 & 37 & 42 & 44 & 51 & 54 & 58 & 66 \\
\hline Max & 72 & 57 & 32 & 53 & 43 & 41 & 44 & 49 & 53 & 59 & 53 & 59 \\
\hline Min & 69 & 60 & 54 & 42 & 38 & 33 & 41 & 42 & 49 & 49 & 55 & 63 \\
\hline
\end{tabular}


Table 13.--Daily water temperatures of Columbia River at Stella, Wash., August 1941 to July 1942

[Once-daily observations--mixed morning and afternoon readings at river mile 56.4]

\begin{tabular}{|c|c|c|c|c|c|c|c|c|c|c|c|c|}
\hline Day & August & September & October & November & December & January & February & March & April & May & June & July \\
\hline 1 & & 63 & 63 & 54 & -- & -- & -- & 41 & 48 & 50 & 57 & -- \\
\hline 2 & & 67 & 61 & 54 & 46 & 34 & 41 & 42 & 50 & 50 & 56 & 67 \\
\hline 3 & & 66 & 62 & 54 & 46 & 34 & 41 & 41 & 49 & 51 & 59 & 68 \\
\hline 4 & & 66 & 60 & 54 & 46 & 34 & 42 & 43 & 51 & 50 & 60 & 68 \\
\hline 5 & & 67 & -- & 54 & 46 & 31 & 43 & 42 & -- & 51 & -- & 66 \\
\hline 6 & & 66 & 60 & 53 & 47 & 32 & 42 & 42 & 51 & 54 & 59 & 66 \\
\hline 7 & & 67 & -- & 54 & 47 & 32 & 42 & 44 & 51 & 54 & 60 & 67 \\
\hline 8 & & 66 & 57 & 53 & 45 & 32 & 42 & 44 & 51 & 54 & 58 & 67 \\
\hline 9 & & 65 & 58 & 52 & 45 & 31 & 43 & 44 & 53 & 54 & 59 & 61 \\
\hline 10 & & 65 & 58 & 52 & 46 & 33 & 43 & 45 & -- & 54 & 59 & 62 \\
\hline 11 & & 63 & 57 & 53 & 44 & 32 & 44 & 45 & 53 & 55 & 58 & 65 \\
\hline 12 & 71 & 65 & 58 & 52 & 42 & 33 & 43 & 43 & 54 & 54 & 59 & 67 \\
\hline 13 & 71 & 64 & 58 & -- & 43 & 34 & 42 & 45 & 52 & 56 & 60 & 65 \\
\hline 14 & 71 & 64 & 55 & 52 & -- & 35 & 42 & 45 & 51 & 56 & 60 & 66 \\
\hline 15 & 71 & 63 & 58 & 51 & 43 & 33 & 44 & 44 & 52 & 55 & 59 & 65 \\
\hline 16 & 72 & 63 & 57 & 50 & 44 & 34 & 42 & 44 & 51 & 58 & 59 & 65 \\
\hline 17 & -- & 63 & 59 & 50 & 44 & 35 & 42 & 45 & 53 & 57 & 57 & 65 \\
\hline 18 & 72 & -- & 57 & 49 & 45 & 35 & 42 & 46 & 53 & 55 & 58 & 65 \\
\hline 19 & 71 & 63 & 57 & 48 & 44 & 33 & 42 & 45 & 54 & 57 & 58 & 68 \\
\hline 20 & 71 & 62 & 55 & 48 & -- & 38 & 41 & 44 & 53 & 58 & 58 & 67 \\
\hline 21 & 72 & 62 & 55 & 47 & 45 & 37 & 41 & 45 & 53 & 58 & 61 & 67 \\
\hline 22 & 72 & 62 & 55 & 46 & 45 & 37 & 40 & 45 & 53 & 58 & 60 & 68 \\
\hline 23 & 73 & 64 & 57 & 45 & 44 & 36 & 41 & 45 & 52 & 59 & 59 & 67 \\
\hline 24 & 72 & 63 & 58 & - & 44 & 37 & 40 & 46 & 52 & 58 & 61 & 69 \\
\hline 25 & 71 & 63 & -- & 46 & 45 & 39 & 42 & 47 & 53 & 58 & 59 & 69 \\
\hline 26 & 70 & 63 & 56 & 46 & 43 & 39 & 41 & 48 & 52 & 58 & 60 & -- \\
\hline 27 & 69 & 62 & 56 & -- & 39 & 39 & 40 & 47 & 51 & 58 & 59 & -- \\
\hline 28 & 70 & 62 & 57 & 45 & - & 41 & 41 & 48 & 52 & 56 & 61 & -- \\
\hline 29 & 70 & 62 & 56 & 45 & 38 & 39 & -- & 49 & 52 & 58 & -- & -- \\
\hline 30 & 69 & 62 & 55 & 46 & 40 & 39 & ----- & 47 & 50 & 57 & 64 & -- \\
\hline 31 & 68 & ------ & 55 & ------ & 38 & 39 & $-\ldots-\cdots$ & 47 & $-\ldots-\cdots$ & 57 &.----- & -- \\
\hline Avg & 71 & 64 & 58 & 50 & 44 & 35 & 42 & 45 & 52 & 55 & 59 & 66 \\
\hline Max & 73 & 67 & 63 & 54 & 47 & 41 & 44 & 49 & 54 & 59 & 64 & 69 \\
\hline Min & 68 & 62 & 55 & 45 & 38 & 31 & 40 & 41 & 18 & 50 & 55 & 51 \\
\hline
\end{tabular}


Table 14.--Daily water temperatures of Columbia River at Eagle Cliff, Wash., August 1941 to July 1942

[Once-daily observations--mostly afternoon readings at river mile 50.6]

\begin{tabular}{|c|c|c|c|c|c|c|c|c|c|c|c|c|}
\hline Day & August & September & October & November & December & January & Febr'uary & March & April & May & June & July \\
\hline 1 & & 68 & 63 & 55 & 47 & 37 & 40 & 42 & 47 & 50 & 56 & 64 \\
\hline 2 & & 67 & 61 & 55 & 47 & 38 & 42 & 41 & 48 & 50 & 57 & 66 \\
\hline 3 & & 66 & 62 & 54 & 46 & 37 & 40 & 42 & 48 & 50 & 57 & 66 \\
\hline 4 & & 66 & 60 & 54 & 45 & 37 & 42 & 42 & 49 & 51 & 58 & 66 \\
\hline 5 & & 66 & 61 & 54 & 46 & 35 & 41 & 41 & 49 & 51. & 59 & 68 \\
\hline 6 & & 65 & 59 & 54 & 48 & 35 & 41 & 42 & 50 & 52 & -- & 68 \\
\hline 7 & & 66 & 59 & 54 & 46 & 35 & 42 & 42 & 50 & 53 & 59 & 66 \\
\hline 8 & & 64 & 59 & 54 & 46 & 33 & 42 & 42 & 50 & 53 & 58 & 67 \\
\hline 9 & & 65 & 58 & 53 & 46 & 35 & 43 & 43 & 50 & 53 & 58 & 66 \\
\hline 10 & & 65 & 58 & 52 & 46 & 33 & 42 & 43 & 52 & -- & 58 & 66 \\
\hline 11 & & 64 & 57 & 53 & 44 & 32 & 43 & 43 & -- & 53 & 58 & 66 \\
\hline 12 & 70 & 64 & 58 & 52 & 44 & 34 & 43 & 43 & 53 & 54 & 58 & 66 \\
\hline 13 & 70 & 64 & 57 & 52 & 45 & 33 & 42 & 44 & 52 & 55 & 59 & 65 \\
\hline 14 & 71 & 62 & 58 & 52 & 44 & 37 & 42 & 44 & 52 & 57 & 58 & 65 \\
\hline 15 & 70 & 61 & 57 & 51 & 45 & 34 & 42 & 44 & 52 & 55 & 58 & 65 \\
\hline 16 & 71 & 63 & 57 & 50 & 45 & 34 & 40 & 44 & 52 & 55 & 58 & -- \\
\hline 17 & -- & 62 & 57 & 50 & 45 & 34 & 43 & 44 & 52 & 56 & 58 & 64 \\
\hline 18 & 72 & 62 & 57 & 49 & 45 & 34 & 43 & 45 & 52 & 56 & 58 & 66 \\
\hline 19 & 72 & 61 & 57 & 48 & 46 & 36 & 43 & 45 & 52 & 55 & 58 & 66 \\
\hline 20 & 70 & 61 & 56 & -- & 47 & 37 & 41 & 45 & 54 & 59 & 58 & 66 \\
\hline 21 & 67 & 60 & 58 & 46 & 45 & 39 & 42 & 44 & 52 & 57 & 58 & 66 \\
\hline 22 & 71 & 60 & 57 & 46 & 45 & 40 & 41 & 44 & 52 & 55 & 59 & 67 \\
\hline 23 & 72 & 61 & 57 & 45 & 45 & 41 & 40 & 44 & 52 & 58 & 60 & 67 \\
\hline 24 & 72 & 61 & 57 & 46 & 45 & 37 & 41 & 44 & 52 & 57 & 60 & 69 \\
\hline 25 & 70 & 62 & 56 & 47 & 45 & 38 & 40 & 44 & 52 & 57 & 59 & 69 \\
\hline 26 & 70 & 62 & 56 & 46 & 44 & 38 & 40 & 45 & 52 & 57 & 59 & -- \\
\hline 27 & 68 & 61 & 56 & 46 & 42 & 38 & 41 & 45 & 50 & 56 & 60 & 70 \\
\hline 28 & 70 & 62 & 56 & 46 & 43 & 40 & 40 & 46 & 51 & 57 & -- & 70 \\
\hline 29 & 69 & 63 & 56 & 45 & 41 & 40 & -- & 46 & 52 & 57 & 62 & 69 \\
\hline 30 & 68 & 63 & 55 & 45 & 41 & 40 & ------ & 46 & 50 & 57 & 62 & 69 \\
\hline 31 & 68 & ------ & 54 & $--\cdots--$ & 39 & 39 & $---\cdots-$ & 47 & $---\cdots$ & 56 & $---\cdots$ & 69 \\
\hline Avs & $7 n$ & 63 & 58 & $5 n$ & 45 & 36 & 42 & 41 & 51 & 55 & 50 & 57 \\
\hline Max & 72 & 68 & 63 & 55 & 48 & 41 & 43 & 47 & 54 & 59 & 62 & 70 \\
\hline Min & 67 & 60 & 54 & 45 & 39 & 32 & 40 & 41 & 47 & 50 & 56 & 64 \\
\hline
\end{tabular}


Table 15. --Daily water temperatures of Columbia River at Wauna, Oreg., November 1940 to August 1942

[Once-daily observations--mixed morning and afternoon readings at river mile 4l.8]

\begin{tabular}{|c|c|c|c|c|c|c|c|c|c|c|c|}
\hline Day & November & December & January & February & March & April & May & June & July & August & September \\
\hline 1 & -- & 48 & 39 & 43 & 46 & 50 & 56 & 61 & 64 & 72 & 68 \\
\hline 2 & -- & 46 & 39 & 43 & 46 & 54 & 56 & 60 & 64 & 72 & 67 \\
\hline 3 & -- & 46 & 40 & 46 & 46 & 52 & 57 & 60 & 64 & 72 & 67 \\
\hline 4 & -- & 46 & 40 & 44 & 46 & 52 & -- & 60 & -- & 68 & 67 \\
\hline 5 & 53 & 46 & 40 & 44 & 45 & 53 & 57 & 59 & 67 & 70 & 67 \\
\hline 6 & 53 & 47 & 39 & 44 & 47 & 52 & 57 & 59 & 66 & 71 & 67 \\
\hline 7 & 53 & 46 & 39 & 44 & 46 & 53 & 57 & 60 & 67 & 71 & -- \\
\hline 8 & 49 & 46 & 39 & 44 & 46 & 53 & 58 & $-\infty$ & 67 & 71 & 65 \\
\hline 9 & 50 & 41 & 39 & 44 & 46 & 54 & 58 & 60 & 67 & 70 & 65 \\
\hline 10 & 50 & 41 & 39 & 44 & 46 & 54 & 57 & 64 & 67 & -- & 64 \\
\hline 11 & 49 & 39 & 40 & 44 & 49 & 54 & -- & 63 & 68 & 68 & 65 \\
\hline 12 & 47 & 39 & 40 & 44 & 49 & 54 & 58 & 63 & 69 & 69 & 65 \\
\hline 13 & 45 & 40 & 40 & 43 & 48 & 54 & 58 & 63 & - & 68 & 65 \\
\hline 14 & 46 & 39 & 40 & 41 & 49 & 54 & 57 & 62 & 74 & 68 & -- \\
\hline 15 & 45 & 38 & 40 & 41 & 49 & 54 & 57 & -- & 73 & 68 & 63 \\
\hline 16 & 44 & 39 & 40 & 43 & -- & 54 & 57 & $\overline{63}$ & 73 & 68 & 64 \\
\hline 17 & 50 & 40 & 42 & 45 & 48 & 54 & 57 & 63 & 73 & 67 & 63 \\
\hline 18 & 51 & 40 & 41 & 41 & 48 & 54 & 57 & 63 & 72 & 67 & 63 \\
\hline 19 & 44 & 40 & 41 & 43 & 49 & 54 & 56 & 63 & 72 & 71 & 63 \\
\hline 20 & 45 & 41 & 44 & 41 & 49 & $=$ & 57 & 63 & 72 & 70 & 61 \\
\hline 21 & 41 & 40 & 43 & 45 & 49 & 54 & 58 & 63 & 72 & 71 & -- \\
\hline 22 & 44 & 41 & 42 & 42 & 50 & 54 & 61 & -- & 73 & 69 & 62 \\
\hline 23 & 43 & 39 & 41 & 43 & -- & 54 & 59 & 63 & 73 & 69 & 63 \\
\hline 24 & 42 & 41 & 42 & 43 & 49 & 55 & 59 & 63 & 73 & - & 62 \\
\hline 25 & 43 & 41 & 42 & 43 & 50 & 56 & -- & 64 & 73 & 68 & 62 \\
\hline 26 & 41 & 41 & 41 & 43 & 50 & 55 & 59 & 64 & 73 & 69 & 62 \\
\hline 27 & 42 & 42 & 44 & 44 & 50 & -- & 59 & 64 & -- & 69 & 61 \\
\hline 28 & 45 & 41 & 44 & 44 & 50 & 56 & 60 & 63 & 72 & 69 & - \\
\hline 29 & 44 & 41 & 43 & -- & 50 & 55 & 61 & 64 & 73 & 69 & 61 \\
\hline 30 & 44 & 41 & 43 & ----- & 49 & 55 & 62 & 64 & 72 & 68 & 61 \\
\hline 31 & $\ldots-\cdots$ & 41 & 43 & ------ & 50 & ----- & 61 & ------ & 72 & -- & ------ \\
\hline Avg & 46 & 42 & 41 & 43 & 48 & 54 & 58 & 62 & 70 & 69 & 64 \\
\hline Max & 53 & 48 & 44 & 46 & 50 & 56 & 62 & 64 & 74 & 72 & 68 \\
\hline Min & 41 & 38 & 39 & 41 & 45 & 50 & 56 & 59 & 64 & 67 & 61 \\
\hline
\end{tabular}


Table 15.--Daily water temperatures of Columbia River at Wauna, Oreg., November 1940 to August 1942--Continued

\begin{tabular}{|c|c|c|c|c|c|c|c|c|c|c|c|}
\hline Day & October & November & December & January & February & March & April & May & June & July & August \\
\hline 1 & 61 & -- & 47 & 40 & 37 & 43 & 46 & 53 & 59 & 64 & 66 \\
\hline 2 & 61 & -- & 47 & 37 & 41 & 43 & 47 & 54 & 59 & 64 & -- \\
\hline 3 & 60 & 55 & 47 & 37 & 40 & 42 & 47 & -- & 59 & 64 & 66 \\
\hline 4 & 61 & 55 & 47 & -- & 40 & 43 & 47 & 54 & 60 & 64 & 66 \\
\hline 5 & 61 & 55 & 47 & 37 & 41 & 44 & $\ldots$ & 55 & 60 & -- & 67 \\
\hline 6 & 61 & 55 & 47 & 37 & 41 & 44 & 47 & 55 & 60 & 64 & -- \\
\hline 7 & 60 & 55 & -- & 37 & 42 & 44 & 48 & 55 & -- & 64 & -- \\
\hline 8 & 60 & 55 & 46 & 37 & -- & -- & 49 & 55 & 59 & 64 & 71 \\
\hline 9 & 60 & -- & 46 & 37 & 42 & 43 & 49 & 55 & 60 & 64 & - \\
\hline 10 & 58 & 55 & 46 & 37 & 43 & 44 & 49 & -- & 60 & 64 & 71 \\
\hline 11 & 58 & 55 & 46 & -- & 43 & 43 & 49 & 54 & 60 & 64 & 71 \\
\hline 12 & -- & 55 & 45 & 35 & 41 & 43 & -- & 55 & 61 & -- & 72 \\
\hline 13 & 57 & 54 & 45 & 35 & 42 & 43 & 50 & 55 & 61 & 64 & 72 \\
\hline 14 & 57 & 53 & -- & 35 & 43 & 44 & 50 & 55 & -- & 64 & 72 \\
\hline 15 & 57 & 53 & 45 & 35 & $\ldots$ & -- & 52 & 55 & 61 & 65 & 72 \\
\hline 16 & 58 & -- & 45 & 36 & 43 & 43 & 52 & 55 & 60 & 65 & -- \\
\hline 17 & 58 & 49 & 45 & 36 & 43 & 44 & 52 & -- & 60 & 65 & 70 \\
\hline 18 & 57 & 49 & 45 & -- & 43 & 44 & 52 & 56 & 60 & 65 & 72 \\
\hline 19 & -- & 49 & 46 & 36 & 43 & 44 & -- & 56 & 60 & -- & 72 \\
\hline 20 & 57 & 48 & 46 & 36 & 43 & 46 & 52 & 56 & 60 & 65 & 72 \\
\hline 21 & 57 & 48 & -- & 36 & 43 & 45 & 52 & 58 & - & 65 & 72 \\
\hline 22 & 57 & 48 & 45 & 36 & 43 & -- & 53 & 58 & 60 & 65 & 72 \\
\hline 23 & 57 & -- & -- & 36 & 43 & 43 & 53 & 58 & 60 & 65 & -- \\
\hline 24 & 57 & 47 & 45 & 36 & 43 & 43 & 53 & -- & 60 & 66 & 71 \\
\hline 25 & 57 & 47 & 44 & 36 & 43 & 44 & 53 & 58 & 59 & 66 & 71 \\
\hline 26 & -- & 47 & 43 & 37 & 43 & 44 & -- & 58 & 60 & -- & 70 \\
\hline 27 & 56 & 46 & 43 & 37 & 42 & 45 & 53 & 58 & 60 & 66 & 70 \\
\hline 28 & 56 & 46 & -- & 37 & 42 & 44 & 53 & 58 & -- & 66 & 69 \\
\hline 29 & 56 & 47 & 41 & 36 & -- & -- & 53 & 58 & 60 & 66 & 69 \\
\hline 30 & 56 & 47 & 41 & 38 & - & 46 & 53 & 59 & 59 & 66 & -- \\
\hline 31 & 54 & - - & 40 & 38 & $-\cdots$ & 46 & $-\cdots$ & -- & $-\cdots$ & 66 & 69 \\
\hline Avg & 53 & 51 & 45 & 37 & $4 ?$ & 44 & 51 & 55 & 50 & 55 & 70 \\
\hline Max & 61 & 55 & 47 & 40 & 43 & 46 & 53 & 59 & 61 & 66 & 72 \\
\hline Min & 54 & 46 & 40 & 35 & 37 & 42 & 46 & 53 & 59 & 64 & 66 \\
\hline
\end{tabular}


Table 16.--Daily water temperatures of Columbia River at Cathlamet, Wash., September 1940 to July 1942

[Once-daily observations--mostly afternoon readings at river mile 39.5]

\begin{tabular}{|c|c|c|c|c|c|c|c|c|c|c|c|c|}
\hline Day & September & Ortober & November & December & January & February & March & April & May & June & July & August \\
\hline 1 & & 64 & 56 & -- & 42 & 43 & 43 & 51 & 59 & -- & 66 & 70 \\
\hline 2 & & 64 & 55 & 46 & 41 & -- & -- & 51 & 59 & -- & 66 & 70 \\
\hline 3 & & -- & -- & -- & 40 & 43 & 43 & 53 & 58 & 60 & 67 & -- \\
\hline 4 & & 64 & 54 & 46 & 40 & 43 & 44 & 53 & 58 & 60 & -- & 70 \\
\hline 5 & & 63 & 53 & 46 & 40 & 43 & -- & 53 & 59 & -- & 68 & 69 \\
\hline 6 & & -- & 53 & 46 & 39 & -- & 45 & -- & 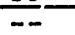 & 61 & -- & 70 \\
\hline 7 & & 62 & 53 & 46 & 39 & 43 & 45 & - & -- & 62 & 69 & 72 \\
\hline 8 & & 63 & 51 & -- & 39 & 43 & 46 & -- & 59 & -- & 70 & -- \\
\hline 9 & & -- & 51 & 45 & 39 & -- & $-\infty$ & -- & 59 & 62 & 71 & 70 \\
\hline 10 & & 62 & -- & 45 & 39 & 43 & 47 & -- & -- & 64 & 70 & -- \\
\hline 11 & & 61 & 50 & 44 & 39 & 43 & 47 & 54 & -- & 64 & 70 & 70 \\
\hline 12 & & 61 & 49 & 43 & -- & 44 & 47 & -- & 59 & 64 & 70 & 71 \\
\hline 13 & & -- & 48 & 41 & -- & 43 & 48 & -- & 59 & 64 & -- & 71 \\
\hline 14 & & 61 & 48 & 41 & 39 & 43 & -- & 54 & 59 & -- & 72 & 71 \\
\hline 15 & & 61 & 47 & -- & 39 & 43 & -- & 54 & 59 & -- & 72 & 71 \\
\hline 16 & & 61 & 46 & -- & 39 & -- & -- & 54 & 59 & 64 & 73 & -- \\
\hline 17 & & 61 & -- & -- & 39 & 43 & 48 & 53 & 59 & 64 & 74 & -- \\
\hline 18 & & 61 & 45 & -- & 40 & 43 & 48 & 53 & -- & 64 & 75 & 71 \\
\hline 19 & & 62 & 44 & -- & -- & 43 & -- & 54 & 57 & 64 & 75 & 72 \\
\hline 20 & 69 & -- & 44 & 37 & 42 & 43 & 48 & -- & 58 & 64 & -- & 71 \\
\hline 21 & 66 & 62 & 44 & 38 & 42 & 43 & -- & 55 & 59 & 64 & 75 & -- \\
\hline 22 & -- & 62 & 43 & -- & -- & 43 & 49 & 56 & 59 & -- & 75 & -- \\
\hline 23 & 66 & 62 & 42 & 40 & 42 & -- & -- & 56 & 59 & 64 & 74 & 72 \\
\hline 24 & 65 & 61 & 43 & 40 & 42 & 43 & -- & -- & 60 & 64 & 74 & -- \\
\hline 25 & 67 & -- & 42 & -- & 42 & 43 & 49 & 57 & -- & 65 & -- & 72 \\
\hline 26 & 65 & 59 & -- & 42 & -- & 43 & 50 & -- & -- & 65 & 73 & 71 \\
\hline 27 & 65 & -- & 42 & 42 & -- & 43 & 50 & -- & 60 & 65 & -- & 70 \\
\hline 28 & 64 & 58 & 42 & 41 & 43 & 44 & 50 & -- & 61 & 65 & 72 & -- \\
\hline 29 & -- & 57 & 43 & -- & 43 & -- & 51 & -- & 61 & -- & 72 & -- \\
\hline 30 & 64 & 57 & 44 & 42 & 43 & $-\infty \ldots$ & -- & 58 & -- & 65 & 72 & 69 \\
\hline 31 & ----- & 56 & ------ & 42 & 43 & ------ & 51 & $-\cdots--$ & 61 & $-\cdots--$ & 71 & -- \\
\hline Avg & & 61 & 47 & 43 & 41 & 43 & 47 & 54 & 59 & 64 & 71 & 71 \\
\hline $\operatorname{Max}$ & & 64 & 56 & 46 & 43 & 44 & 51 & 58 & 61 & 65 & 75 & 72 \\
\hline Min & & 56 & 42 & 37 & 39 & 43 & 43 & 51 & 57 & 60 & 66 & 69 \\
\hline
\end{tabular}


Table 16.--Daily water temperatures of Columbia River at Cathlamet, Wash., September 1940 to July $1942--C o n t i n u e d$

\begin{tabular}{|c|c|c|c|c|c|c|c|c|c|c|c|}
\hline Day & September & Otober & November & December & January & February & March & April & May & June & July \\
\hline 1 & 68 & 63 & -- & 46 & 39 & -- & 42 & 49 & 51 & 57 & 64 \\
\hline 2 & 67 & 63 & -- & 46 & 38 & 40 & 42 & 49 & 51 & 57 & 65 \\
\hline 3 & 66 & -- & 54 & 46 & 37 & 41 & 41 & 49 & 50 & -- & 66 \\
\hline 4 & 66 & 62 & 54 & 46 & -- & 41 & 42 & 50 & 52 & 58 & -- \\
\hline 5 & 66 & - & 54 & 46 & 37 & - & $=$ & $\ldots$ & 52 & 59 & 67 \\
\hline 6 & 66 & 61 & 54 & 46 & 36 & - & 42 & 51 & 53 & 59 & 67 \\
\hline 7 & -- & 61 & 54 & -- & 34 & -- & -- & 51 & 54 & -- & 67 \\
\hline 8 & 66 & 60 & 53 & 46 & 34 & 42 & 43 & 51 & - & 59 & -- \\
\hline 9 & -- & 59 & -- & 46 & 33 & 42 & 44 & 51 & 54 & 59 & 67 \\
\hline 10 & -- & 58 & 52 & -- & 33 & - & 44 & 53 & -- & 60 & 67 \\
\hline 11 & - & 58 & 52 & 45 & -- & 42 & 44 & 53 & 56 & 59 & 67 \\
\hline 12 & -- & -- & 52 & 45 & 34 & -- & 44 & -- & 55 & 59 & 66 \\
\hline 13 & -- & 57 & 52 & 45 & 35 & 43 & 45 & 53 & 57 & 60 & -- \\
\hline 14 & -- & 57 & 52 & -- & 35 & 43 & 45 & 53 & 56 & 59 & 66 \\
\hline 15 & $\ldots$ & 57 & 51 & 44 & 35 & -- & -- & -- & -- & 59 & 65 \\
\hline 16 & 64 & 57 & - & 44 & 35 & 43 & 45 & 53 & 56 & 59 & 65 \\
\hline 17 & 64 & 57 & -- & -- & -- & 42 & 46 & 53 & -- & 58 & 65 \\
\hline 18 & 63 & 57 & 50 & 45 & -- & 42 & 46 & 53 & 56 & -- & 65 \\
\hline 19 & 63 & -- & 49 & 46 & 36 & -- & 46 & 53 & 56 & 59 & 66 \\
\hline 20 & 63 & 57 & -- & 46 & 36 & -- & 47 & 54 & 57 & 59 & 66 \\
\hline 21 & -- & 57 & 48 & -- & 36 & 41 & 45 & 53 & 57 & -- & 66 \\
\hline 22 & 63 & -- & 47 & 45 & 36 & 41 & -- & 53 & 57 & 60 & 67 \\
\hline 23 & 63 & 57 & -- & 45 & 36 & 41 & 46 & 53 & 58 & -- & 68 \\
\hline 24 & 63 & 56 & 46 & 45 & 36 & 41 & 46 & 53 & -- & 60 & 68 \\
\hline 25 & 63 & 56 & 46 & 44 & $=$ & -- & 46 & 53 & 58 & 60 & 69 \\
\hline 26 & 63 & 56 & 46 & 44 & -- & 41 & 46 & -- & 58 & 60 & -- \\
\hline 27 & 63 & 56 & 46 & 42 & 38 & 41 & 46 & 52 & 57 & 60 & 69 \\
\hline 28 & -- & 55 & 46 & -- & 39 & 41 & 46 & 52 & 58 & 61 & 69 \\
\hline 29 & 63 & -- & 46 & 41 & 39 & -- & -- & 52 & 58 & 62 & 69 \\
\hline 30 & 63 & 55 & -- & 41 & 39 & ------ & 48 & 51 & 57 & 62 & 69 \\
\hline 31 & $-\cdots-$ & 55 & ---- & 40 & 39 & - - - - & 48 & - - - - & 57 & --- & 69 \\
\hline $\overrightarrow{\text { Avg }}$ & 04 & 58 & 5o & $4 j$ & 50 & 42 & 45 & 52 & 50 & sy & 67 \\
\hline $\operatorname{Max}$ & 68 & 63 & 54 & 46 & 39 & 43 & 48 & 54 & 58 & 62 & 69 \\
\hline Min & 63 & 55 & 46 & 40 & 33 & 40 & 41 & 49 & 50 & 57 & 64 \\
\hline
\end{tabular}


Table 17. - Daily water temperatures of Columbia River at Skamokawa, Wash., November 1940 to July 1942 [Once-daily observations-mixed morning and afternoon readings at river mile 33 ]

\begin{tabular}{|c|c|c|c|c|c|c|c|c|c|c|c|}
\hline$\overline{\text { Day }}$ & November & December & January & February & March & April & May & June & July & August & September \\
\hline$-\frac{j}{1}$ & & 45 & 41 & 44 & 44 & 53 & 59 & 62 & 65 & -- & 66 \\
\hline 2 & & 45 & 40 & 43 & 44 & 53 & 57 & 59 & 64 & 67 & 66 \\
\hline 3 & & 46 & 40 & 43 & 44 & 53 & 55 & 59 & 65 & -- & 64 \\
\hline 4 & & 46 & 40 & 43 & 44 & 53 & -- & 63 & 65 & 68 & 65 \\
\hline 5 & & 47 & 40 & 43 & 44 & 53 & 56 & 62 & 68 & 68 & 66 \\
\hline 6 & & 46 & 40 & 43 & 46 & 53 & 57 & 60 & 67 & 71 & 64 \\
\hline 7 & 52 & 46 & 40 & 43 & 45 & 53 & 58 & 60 & 69 & 66 & -- \\
\hline 8 & 52 & - & 40 & 44 & 46 & 53 & 58 & 60 & 68 & 67 & 64 \\
\hline 9 & 51 & 43 & 41 & 43 & 46 & 53 & 60 & 61 & 66 & 68 & 64 \\
\hline 10 & 48 & 44 & 40 & 44 & 47 & 54 & 60 & 64 & 67 & -- & 64 \\
\hline 11 & 49 & 42 & 40 & 43 & 49 & 53 & 60 & 64 & 66 & 65 & 63 \\
\hline 12 & 46 & 42 & - & 43 & 48 & 53 & 59 & 63 & 68 & 66 & 64 \\
\hline 13 & 46 & 39 & 40 & 43 & 48 & - & 58 & 63 & - & 69 & 63 \\
\hline 14 & 47 & 40 & 41 & 43 & 47 & 52 & 58 & 63 & 71 & 66 & - \\
\hline 15 & 46 & 40 & 40 & 42 & 47 & 53 & 58 & 63 & 70 & 67 & 64 \\
\hline 16 & 46 & 39 & 39 & 42 & 47 & 52 & 58 & 63 & 70 & 66 & 63 \\
\hline 17 & 45 & 39 & 41 & 42 & 48 & 52 & 58 & 63 & 70 & -- & 63 \\
\hline 18 & 42 & 40 & 41 & 43 & 47 & 52 & 58 & 63 & 67 & 69 & 63 \\
\hline 19 & 43 & 39 & 42 & 43 & 48 & 53 & 57 & 63 & 68 & 70 & 62 \\
\hline 20 & 43 & 40 & 42 & 42 & 48 & 54 & 58 & 62 & - & 69 & 61 \\
\hline 21 & 43 & 40 & 42 & 42 & 49 & 54 & 59 & 64 & 72 & 69 & -- \\
\hline 22 & 44 & 40 & 40 & 42 & 48 & 53 & 60 & 63 & 72 & 69 & 61 \\
\hline 23 & 41 & 40 & 41 & 42 & 49 & 53 & 60 & 64 & 72 & 68 & 59 \\
\hline 24 & 43 & 40 & 41 & 43 & 48 & 55 & 60 & 62 & 70 & 71 & 57 \\
\hline 25 & 43 & 41 & 41 & 43 & 49 & 57 & 60 & 64 & 70 & 68 & 61 \\
\hline 26 & 43 & 42 & -- & 43 & 49 & 57 & 60 & 63 & 72 & 68 & 61 \\
\hline 27 & 43 & 42 & 42 & 43 & 52 & 57 & 60 & 63 & - & 69 & 61 \\
\hline 28 & 43 & 42 & 42 & 43 & 51 & 59 & 60 & 64 & 69 & 68 & -- \\
\hline 29 & 45 & 42 & 43 & - & 53 & 60 & 60 & 63 & 71 & 68 & 62 \\
\hline 30 & 44 & 41 & 43 & ------ & 52 & 58 & 60 & 64 & 71 & 66 & 62 \\
\hline 31 & ------ & 42 & 43 & ------ & 53 & ------ & 60 & $----n$ & 64 & 66 & $----n$ \\
\hline Avg & 45 & 42 & 41 & 43 & 48 & 54 & 59 & 62 & 68 & 68 & 63 \\
\hline Max & 52 & 47 & 43 & 44 & 53 & 60 & 60 & 64 & 72 & 71 & 66 \\
\hline Min & 41 & 39 & 39 & 42 & 44 & 52 & 55 & 59 & 64 & 65 & 57 \\
\hline
\end{tabular}


Table 17.--Daily water temperatures of Columbia River at Skamokawa, Wash., November 1940 to July $1942--C o n$.

\begin{tabular}{|c|c|c|c|c|c|c|c|c|c|c|}
\hline Day & October & November & December & January & February & March & April & May & June & July \\
\hline 1 & 61 & 58 & 47 & 37 & -- & 40 & 48 & 50 & 55 & 66 \\
\hline 2 & 62 & -- & 47 & 36 & 42 & 42 & 49 & 49 & 59 & 66 \\
\hline 3 & 61 & 55 & 45 & 37 & 42 & 41 & 49 & -- & 58 & 66 \\
\hline 4 & 61 & 54 & 47 & -- & 42 & 42 & 52 & 51 & 60 & 66 \\
\hline 5 & -- & 54 & 46 & 36 & 43 & 43 & -- & 54 & 60 & - \\
\hline 6 & 59 & 53 & 49 & 36 & 43 & 43 & 51 & 54 & 59 & 67 \\
\hline 7 & 58 & 53 & -- & 33 & 44 & 46 & 50 & 54 & -- & 66 \\
\hline 8 & 59 & 54 & 45 & 35 & -- & -- & 50 & 54 & 59 & 67 \\
\hline 9 & 56 & -- & 46 & 35 & 43 & 44 & 52 & 54 & 57 & 68 \\
\hline 10 & 59 & 53 & 46 & 34 & 43 & 45 & 52 & -- & 59 & 68 \\
\hline 11 & 55 & 52 & 44 & -- & 44 & 44 & 54 & 54 & 59 & 68 \\
\hline 12 & -- & 52 & 43 & 34 & 42 & 43 & -- & 54 & 60 & -- \\
\hline 13 & 58 & 52 & 43 & 36 & 41 & 44 & 52 & 56 & 58 & 66 \\
\hline 14 & 55 & 52 & -- & 37 & 43 & 46 & 53 & 55 & -- & 66 \\
\hline 15 & 57 & 50 & 45 & 36 & -- & - & 54 & 56 & 59 & 65 \\
\hline 16 & 56 & -- & 44 & 36 & 43 & 46 & -- & 55 & 59 & 65 \\
\hline 17 & 55 & 49 & 43 & 37 & 43 & 45 & -- & -- & 59 & 64 \\
\hline 18 & 55 & 48 & 45 & -- & 42 & 45 & -- & 56 & 59 & 66 \\
\hline 19 & -- & 48 & 46 & 36 & 41 & 44 & 54 & 47 & 59 & -- \\
\hline 20 & 52 & 48 & 46 & 36 & 42 & 49 & 55 & 59 & 59 & 68 \\
\hline 21 & 59 & 46 & -- & 39 & 40 & 45 & 54 & 58 & -- & 67 \\
\hline 22 & 56 & 46 & 45 & 37 & -- & -- & 52 & 57 & 61 & 66 \\
\hline 23 & 55 & -- & 44 & 38 & 41 & 44 & 53 & 59 & 60 & 68 \\
\hline 24 & 56 & 45 & 44 & 40 & 41 & 45 & 51 & -- & 59 & 66 \\
\hline 25 & 55 & 46 & 44 & -- & 41 & 46 & 51 & 58 & 59 & 68 \\
\hline 26 & -- & 46 & 42 & 41 & 40 & 48 & -- & 58 & 61 & -- \\
\hline 27 & 55 & 45 & 40 & 40 & 41 & 46 & 51 & 57 & 61 & 67 \\
\hline 28 & 55 & 46 & -- & 39 & 42 & 46 & 52 & 58 & -- & 69 \\
\hline 29 & 56 & 46 & 40 & 40 & -- & -- & 52 & 57 & 63 & 67 \\
\hline 30 & 54 & -- & 38 & 40 & ------ & 48 & 50 & 59 & 64 & 68 \\
\hline 31 & 54 & $--\cdots--$ & 38 & 40 & ------ & 48 & ------ & -- & $---n-n$ & 68 \\
\hline$\overline{\mathrm{Avg}}$ & 57 & 50 & 11 & $3 ?$ & 12 & 45 & 52 & 55 & 59 & 57 \\
\hline Max & 62 & 58 & 49 & 41 & 44 & 49 & 55 & 59 & 64 & 69 \\
\hline Min & 52 & 45 & 38 & 34 & 40 & 40 & 48 & 49 & 55 & 64 \\
\hline
\end{tabular}


Table 18.--Daily water temperatures of Columbia River at Altoona, Wash., September 1940 to June 1942

[Once-daily observations-mixed morning and afternoon readings at river mile 24]

\begin{tabular}{|c|c|c|c|c|c|c|c|c|c|c|c|c|}
\hline Day & September & October & November & December & January & February & March & April & Ma.y & June & July & August \\
\hline 1 & & 64 & 55 & 45 & 43 & 44 & 44 & 53 & 59 & 61 & 65 & 72 \\
\hline 2 & & 63 & 55 & 46 & 42 & -- & 44 & 53 & 59 & 61 & 66 & 70 \\
\hline 3 & & 63 & -- & 46 & 41 & 44 & 45 & 54 & 59 & 61 & 66 & 69 \\
\hline 4 & & 63 & 54 & 46 & 41 & 44 & 45 & 54 & -- & 61 & 66 & -- \\
\hline 5 & & 63 & 54 & 46 & 41 & 44 & 46 & -- & 59 & 61 & - & 69 \\
\hline 6 & & 63 & 53 & 46 & 41 & 44 & 46 & 54 & 59 & 61 & 67 & 71 \\
\hline 7 & & 63 & 53 & 46 & 41 & 44 & 46 & 54 & 59 & 61 & 68 & 70 \\
\hline 8 & & 63 & 52 & 46 & 41 & 44 & 46 & 54 & 59 & 62 & 69 & 70 \\
\hline 9 & & 62 & 51 & 46 & 42 & 44 & 46 & 54 & 59 & 62 & 70 & 70 \\
\hline 10 & & 61 & 50 & 46 & 42 & 45 & 46 & 54 & 59 & 63 & 71 & 70 \\
\hline 11 & & 61 & 50 & -- & 41 & 45 & 47 & 54 & 59 & 64 & -- & 70 \\
\hline 12 & & 61 & 49 & -- & 41 & 45 & 48 & 54 & 60 & 64 & -- & 70 \\
\hline 13 & & 61 & 48 & 43 & 41 & 45 & 48 & 54 & 60 & 64 & 72 & 70 \\
\hline 14 & & 61 & 48 & 42 & 41 & 44 & 49 & 54 & 60 & -- & 72 & 71 \\
\hline 15 & & 61 & 48 & 42 & 41 & 44 & 49 & 54 & 60 & 64 & 72 & 70 \\
\hline 16 & & 61 & 48 & 41 & 40 & 43 & 49 & 54 & 59 & 64 & 73 & 70 \\
\hline 17 & & 61 & 47 & 41 & 40 & 43 & 49 & 54 & 59 & 64 & 73 & 70 \\
\hline 18 & & 61 & 46 & 41 & 40 & 43 & 49 & 54 & 58 & 64 & 73 & 70 \\
\hline 19 & 66 & 61 & 46 & 41 & 40 & 43 & 49 & 54 & 58 & 64 & 73 & 71 \\
\hline 20 & 66 & 61 & 45 & 41 & 41 & 43 & 49 & 54 & 59 & 64 & -- & 71 \\
\hline 21 & 68 & 61 & 45 & 41 & 42 & 44 & 49 & 55 & 59 & 64 & 73 & 71 \\
\hline 22 & 68 & 59 & 45 & 40 & 42 & 44 & 49 & 55 & 59 & 64 & 73 & 71 \\
\hline 23 & 66 & 60 & 45 & 40 & 43 & 44 & 49 & 55 & 59 & 64 & 73 & -- \\
\hline 24 & 65 & 60 & 44 & 41 & 43 & 44 & 49 & 56 & 60 & 64 & 73 & -- \\
\hline 25 & 66 & 60 & 44 & 41 & 43 & -- & 49 & 57 & 60 & 64 & 73 & 70 \\
\hline 26 & 66 & 59 & 44 & 42 & 43 & 44 & 50 & 58 & 61 & 65 & 73 & 70 \\
\hline 27 & 66 & 59 & 44 & 43 & 43 & 44 & 51 & -- & 61 & 65 & 72 & 70 \\
\hline 28 & 66 & 58 & 44 & 43 & 43 & 44 & 51 & 58 & 61 & 65 & 72 & 70 \\
\hline 29 & 65 & 57 & 43 & 43 & 43 & -- & 53 & 59 & 61 & -- & 72 & 70 \\
\hline 30 & 65 & 57 & 43 & 43 & 43 & ------ & 54 & 59 & 61 & 65 & 72 & 69 \\
\hline 31 & ------ & 57 & ------ & 43 & 43 & ------ & 54 & $\ldots-\cdots$ & 61 & ------ & 72 & 69 \\
\hline $\begin{array}{l}\text { Avg } \\
\text { Max } \\
\text { Min }\end{array}$ & & $\begin{array}{l}61 \\
64 \\
57\end{array}$ & $\begin{array}{l}48 \\
55 \\
43\end{array}$ & $\begin{array}{l}43 \\
46 \\
40\end{array}$ & $\begin{array}{l}42 \\
43 \\
40\end{array}$ & $\begin{array}{l}44 \\
45 \\
43\end{array}$ & $\begin{array}{l}48 \\
54 \\
44\end{array}$ & $\begin{array}{l}55 \\
59 \\
53\end{array}$ & $\begin{array}{l}60 \\
61 \\
58\end{array}$ & $\begin{array}{l}63 \\
65 \\
61\end{array}$ & $\begin{array}{l}71 \\
73 \\
65\end{array}$ & $\begin{array}{l}70 \\
72 \\
69\end{array}$ \\
\hline
\end{tabular}


Table 18.--Daily water temperatures of Columbia River at Altoona, Wash., September 1940 to June $1942--C o n$.

\begin{tabular}{|c|c|c|c|c|c|c|c|c|c|c|}
\hline Day & September & October & November & December & January & February & March & April & May & June \\
\hline 1 & 69 & 61 & 56 & 46 & 40 & 40 & 43 & 47 & 54 & 59 \\
\hline 2 & 68 & 62 & 56 & 46 & 40 & 40 & 43 & 48 & 54 & 59 \\
\hline 3 & -- & 61 & - & 46 & 39 & 41 & 43 & 48 & 54 & 59 \\
\hline 4 & 67 & 61 & 55 & -- & 37 & 41 & 43 & 49 & 54 & 60 \\
\hline 5 & 66 & -- & 54 & 46 & 37 & -- & 43 & -- & 54 & 60 \\
\hline 6 & 66 & 61 & 54 & 46 & -- & 41 & 43 & 49 & 54 & 60 \\
\hline 7 & 66 & 61 & 54 & 46 & 36 & 41 & 43 & 49 & -- & 60 \\
\hline 8 & 66 & 60 & 54 & 46 & 36 & 42 & 43 & 50 & -- & 60 \\
\hline 9 & 66 & 60 & 54 & -- & 36 & - & 43 & 51 & -- & 60 \\
\hline 10 & 66 & 59 & 54 & 46 & -- & 42 & 43 & -- & 54 & 60 \\
\hline 11 & 66 & 59 & 54 & 46 & 36 & 43 & 43 & -- & -- & -- \\
\hline 12 & 65 & -- & 54 & 46 & 36 & 43 & 44 & 51 & 54 & 61 \\
\hline 13 & 64 & 59 & 54 & 46 & 36 & 43 & 44 & 51 & 54 & 61 \\
\hline 14 & -- & 59 & 54 & 46 & 36 & 44 & 44 & 52 & 56 & 61 \\
\hline 15 & 64 & 59 & 53 & 45 & -- & 44 & 45 & 52 & 56 & 61 \\
\hline 16 & -- & 59 & 52 & 45 & 36 & 44 & 45 & 53 & 56 & 61 \\
\hline 17 & 63 & 58 & 51 & 45 & 36 & 44 & 46 & 53 & -- & -- \\
\hline 18 & -- & 58 & 51 & 45 & -- & 44 & 46 & 54 & -- & 61 \\
\hline 19 & 61 & -- & -- & 45 & 36 & 44 & 46 & 54 & 56 & 61 \\
\hline 20 & 61 & 58 & 50 & 45 & 36 & 44 & 46 & -- & 56 & 62 \\
\hline 21 & 61 & -- & -- & 45 & 36 & 44 & 46 & 54 & 56 & 62 \\
\hline 22 & 61 & 57 & 49 & 45 & 36 & 44 & 46 & 54 & 56 & 62 \\
\hline 23 & -- & 57 & 49 & 45 & 36 & 44 & -- & 54 & -- & 62 \\
\hline 24 & 61 & 57 & 48 & - & -- & 44 & 46 & 54 & -- & 62 \\
\hline 25 & 61 & 57 & 47 & -- & 37 & 44 & 46 & 54 & 57 & 62 \\
\hline 26 & 61 & 56 & 46 & 44 & 38 & 43 & 46 & 54 & 58 & 62 \\
\hline 27 & 61 & 56 & - & 44 & -- & 43 & 46 & 54 & 58 & 62 \\
\hline 28 & 61 & 56 & 46 & 43 & 38 & 43 & 46 & 54 & 59 & 62 \\
\hline 29 & 61 & 56 & 46 & 43 & 38 & -- & 47 & 54 & 59 & 62 \\
\hline 30 & 61 & 56 & 46 & 43 & 39 & $---n$ & 47 & 54 & 59 & 62 \\
\hline 31 & $----\cdot-$ & 56 & $-\cdots--$ & 42 & 39 & ----- & 47 & ------ & 59 & ------ \\
\hline Avg & 64 & 58 & 52 & 45 & s: & 43 & 45 & j2 & so & 61 \\
\hline $\operatorname{Max}$ & 69 & 62 & 56 & 46 & 40 & 44 & 47 & 54 & 59 & 62 \\
\hline Min & 61 & 56 & 46 & 42 & 36 & 40 & 43 & 47 & 54 & 59 \\
\hline
\end{tabular}


Table 19.--Daily water temperatures of Columbia River at Astoria (Tongue Point), Oreg., November 1940 to June 1942 [Once-daily observations--mostly afternoon readings at river mile 18.2]

\begin{tabular}{|c|c|c|c|c|c|c|c|c|c|c|c|}
\hline$\overline{\text { Day }}$ & November & December & January & February & March & April & May & June & July & August & September \\
\hline 1 & 55 & 45 & 41 & 45 & 46 & 54 & 58 & 61 & 68 & 68 & 64 \\
\hline 2 & 54 & 45 & 40 & 45 & 46 & 53 & 56 & 61 & 66 & 66 & 66 \\
\hline 3 & 54 & 46 & 39 & 44 & 46 & 54 & 57 & 62 & 67 & 67 & 64 \\
\hline 4 & 53 & 46 & 39 & 45 & 46 & 54 & 57 & 62 & 68 & 68 & 64 \\
\hline 5 & 52 & 46 & 41 & 46 & 46 & 53 & 56 & 61 & 68 & 70 & 65 \\
\hline 6 & 53 & 46 & 42 & 46 & 48 & 52 & 57 & 61 & 69 & 69 & 65 \\
\hline 7 & 52 & 46 & 43 & 46 & 48 & 54 & 57 & 61 & 69 & 68 & 64 \\
\hline 8 & 50 & 46 & 43 & 46 & 48 & 54 & 58 & 61 & 69 & 68 & 64 \\
\hline 9 & 50 & 45 & 43 & 45 & 48 & 53 & 59 & 61 & 68 & 68 & 64 \\
\hline 10 & 48 & 44 & 43 & 46 & 49 & 54 & 61 & 63 & 68 & 66 & 64 \\
\hline 11 & 48 & 44 & 43 & 46 & 49 & 54 & 61 & 64 & 68 & 68 & 64 \\
\hline 12 & 48 & 43 & 43 & 46 & 51 & 54 & 60 & 66 & 68 & 69 & 64 \\
\hline 13 & 47 & 43 & 43 & 45 & 49 & 54 & 59 & 64 & -- & 68 & 63 \\
\hline 14 & 47 & 40 & 43 & 45 & 49 & 54 & 58 & 63 & 75 & 68 & -- \\
\hline 15 & 47 & 41 & 41 & 44 & 50 & 52 & 59 & - & 73 & 68 & 64 \\
\hline 16 & 47 & 41 & 42 & 44 & 50 & 52 & 58 & 64 & 72 & 68 & 64 \\
\hline 17 & 47 & 43 & 43 & 45 & 50 & 54 & 57 & 64 & 72 & -- & 63 \\
\hline 18 & 46 & 43 & 43 & 45 & 49 & 54 & 57 & 63 & 73 & 69 & 63 \\
\hline 19 & 46 & 43 & 40 & 45 & 50 & 54 & 57 & 62 & 72 & 70 & 62 \\
\hline 20 & 45 & 43 & 41 & 45 & 49 & 55 & 59 & 63 & -- & 68 & 61 \\
\hline 21 & 44 & 43 & 41 & 45 & 49 & 55 & 61 & 63 & 72 & 66 & -- \\
\hline 22 & 43 & 42 & 41 & 45 & 49 & 56 & 63 & 63 & 70 & 68 & 63 \\
\hline 23 & 43 & 42 & 43 & 45 & 49 & 56 & 64 & 64 & 72 & 68 & 63 \\
\hline 24 & 45 & 42 & 44 & 46 & 50 & 56 & 60 & 64 & 68 & -- & 63 \\
\hline 25 & 44 & 42 & 45 & 46 & 50 & 57 & 61 & 64 & 70 & 66 & 62 \\
\hline 26 & 43 & 41 & 43 & 45 & 52 & 58 & 59 & 64 & 68 & 67 & 62 \\
\hline 27 & 44 & 42 & 44 & 46 & 52 & 57 & 59 & 65 & -- & 68 & 62 \\
\hline 28 & 46 & 43 & 45 & 46 & 53 & 58 & 60 & 66 & 69 & 68 & -- \\
\hline 29 & 46 & 43 & 44 & -- & 54 & 59 & 63 & - & 70 & 68 & 61 \\
\hline 30 & 44 & 42 & 43 & $---\infty-$ & 54 & 58 & 61 & 66 & 70 & 66 & 61 \\
\hline 31 & $----\infty$ & 41 & 45 & ------ & 54 & ------ & 61 & ----- & 70 & -- & $---\infty-$ \\
\hline Avg & 48 & 43 & 42 & 45 & 49 & 55 & 59 & 63 & 70 & 68 & 63 \\
\hline Max & 55 & 46 & 45 & 46 & 54 & 59 & 64 & 66 & 75 & 70 & 66 \\
\hline Min & 43 & 40 & 39 & 44 & 46 & 52 & 56 & 61 & 66 & 66 & 61 \\
\hline
\end{tabular}


Table 19.--Daily water temperatures of Columbia River at Astoria (Tongue Point), Oreg., November 1940 to June 1942--Continued

\begin{tabular}{|c|c|c|c|c|c|c|c|c|c|}
\hline Day & October & November & December & January & February & March & April & May & June \\
\hline 1 & 61 & 54 & 48 & 36 & 43 & -- & 48 & 50 & 55 \\
\hline 2 & 61 & 54 & 49 & 36 & 43 & 45 & 48 & 50 & 57 \\
\hline 3 & 61 & 54 & 46 & 37 & 43 & 43 & 50 & 52 & 57 \\
\hline 4 & 61 & 54 & 46 & 36 & 42 & 43 & 49 & 51 & 57 \\
\hline 5 & 60 & 54 & 46 & 36 & 41 & 43 & 51 & 50 & 59 \\
\hline 6 & 60 & 54 & 46 & 36 & 43 & 43 & 50 & 52 & 59 \\
\hline 7 & 59 & 54 & -- & 36 & 43 & 44 & 50 & 53 & 59 \\
\hline 8 & 59 & 54 & 46 & 36 & 43 & 45 & 49 & 54 & 58 \\
\hline 9 & 59 & 54 & 46 & 36 & 43 & 46 & 50 & 53 & 57 \\
\hline 10 & 59 & 54 & 46 & 36 & 44 & 46 & 52 & -- & 57 \\
\hline 11 & 58 & 54 & 46 & -- & 44 & 44 & 52 & 52 & 58 \\
\hline 12 & -- & 52 & 45 & 35 & 43 & 43 & -- & 54 & 59 \\
\hline 13 & 57 & 54 & 45 & 36 & 43 & 43 & 52 & 54 & 60 \\
\hline 14 & 58 & 53 & -- & 36 & 43 & 43 & 52 & 55 & -- \\
\hline 15 & 58 & 52 & 46 & 36 & -- & -- & 52 & 56 & 58 \\
\hline 16 & 58 & -- & 45 & 37 & 43 & 43 & 52 & 56 & 57 \\
\hline 17 & 58 & 50 & 44 & 38 & 43 & 45 & 53 & -- & 58 \\
\hline 18 & 57 & 49 & 46 & - & 43 & 45 & 51 & 56 & 57 \\
\hline 19 & $-\infty$ & 49 & 45 & 37 & 43 & 45 & -- & 57 & 57 \\
\hline 20 & 58 & 48 & 45 & 37 & 43 & 45 & 53 & 57 & 58 \\
\hline 21 & 58 & 46 & -- & 38 & 43 & 46 & 53 & 58 & -- \\
\hline 22 & 57 & 46 & 46 & 38 & -- & -- & 52 & 57 & 59 \\
\hline 23 & 57 & -- & 45 & 38 & 43 & 46 & 51 & 56 & 60 \\
\hline 24 & 56 & 48 & 45 & 39 & 43 & 46 & 52 & -- & 59 \\
\hline 25 & 56 & 47 & 44 & -- & 43 & 44 & 52 & 58 & 59 \\
\hline 26 & -- & 46 & 42 & 42 & 43 & 45 & -- & 57 & 59 \\
\hline 27 & 55 & 46 & 41 & 41 & 43 & 45 & 51 & 57 & 59 \\
\hline 28 & 55 & 46 & -- & 41 & 43 & 46 & 50 & 57 & -- \\
\hline 29 & 55 & 48 & 41 & 40 & -- & -- & 52 & 57 & 61 \\
\hline 30 & 54 & - & 41 & 41 & $--\infty--$ & 48 & 52 & 58 & 64 \\
\hline 31 & 54 & ------ & 38 & 41 & $---\cdots$ & 48 & ------ & -- & ------ \\
\hline$\overline{\mathrm{Avg}}$ & 58 & 51 & 45 & 38 & 43 & 45 & 51 & 55 & 53 \\
\hline Max & 61 & 54 & 49 & 42 & 44 & 48 & 53 & 58 & 64 \\
\hline Min & 54 & 46 & 38 & 35 & 41 & 43 & 48 & 50 & 55 \\
\hline
\end{tabular}


Table 20.--Daily water temperatures of Columbia River at Fort Stevens, Oreg., August 1941 to January 1942 and June, July 1942 [Once-daily observations--mixed morning and afternoon readings at river mile 6.7]

\begin{tabular}{|c|c|c|c|c|c|c|c|c|c|c|c|c|}
\hline$\overline{D a y}$ & August & September & October & November & December & January & February & March & April & May & June & July \\
\hline$\frac{1}{1}$ & & -- & 62 & 55 & 51 & 38 & & & & & 60 & 64 \\
\hline 2 & & 71 & 62 & -- & 52 & 37 & & & & & 59 & 68 \\
\hline 3 & & 65 & 59 & 55 & -- & 40 & & & & & 59 & 68 \\
\hline 4 & & 64 & 60 & 55 & 49 & -- & & & & & 61 & 66 \\
\hline 5 & & 65 & -- & 57 & 49 & 37 & & & & & 64 & -- \\
\hline 6 & & 66 & -- & 56 & -- & - & & & & & - & 68 \\
\hline 7 & & -- & 57 & 56 & -- & 38 & & & & & - & 69 \\
\hline 8 & & 65 & 58 & 55 & 47 & -- & & & & & 59 & 68 \\
\hline 9 & & 64 & 57 & -- & -- & 41 & & & & & 59 & 68 \\
\hline 10 & & -- & 60 & 54 & 49 & 40 & & & & & -- & 68 \\
\hline 11 & & 64 & 58 & -- & 47 & - & & & & & 59 & 66 \\
\hline 12 & & 66 & -- & 55 & -- & -- & & & & & 62 & -- \\
\hline 13 & & 63 & 59 & 54 & 46 & 40 & & & & & 63 & 69 \\
\hline 14 & & -- & 62 & 55 & -- & 40 & & & & & -- & 65 \\
\hline 15 & & 65 & -- & 53 & 50 & 40 & & & & & 60 & 68 \\
\hline 16 & & 65 & 59 & -- & 49 & 42 & & & & & 59 & 66 \\
\hline 17 & & 64 & 62 & 52 & 48 & 43 & & & & & 61 & 68 \\
\hline 18 & 67 & 63 & 58 & 52 & 51 & -- & & & & & 61 & 68 \\
\hline 19 & 68 & -- & -- & 51 & 48 & -- & & & & & 60 & -- \\
\hline 20 & 67 & 62 & 59 & -- & 48 & 40 & & & & & 63 & 68 \\
\hline 21 & 66 & -- & 58 & 48 & -- & 41 & & & & & -- & 68 \\
\hline 22 & 64 & 64 & 62 & 47 & -- & -- & & & & & 63 & 68 \\
\hline 23 & 66 & 63 & -- & -- & 45 & 41 & & & & & 63 & 68 \\
\hline 24 & -- & 58 & 57 & 50 & 44 & 42 & & & & & 61 & 69 \\
\hline 25 & 58 & 60 & 56 & 48 & -- & -- & & & & & 61 & 68 \\
\hline 26 & -- & 62 & -- & 48 & 43 & 46 & & & & & 60 & - \\
\hline 27 & 63 & -- & 56 & -- & 41 & -- & & & & & 62 & 67 \\
\hline 28 & 65 & -- & 56 & 49 & -- & 47 & & & & & -- & 64 \\
\hline 29 & 60 & 63 & -- & -- & 41 & -- & & & & & 65 & 64 \\
\hline 30 & 65 & 63 & 55 & -- & 44 & 42 & & & & & 66 & 63 \\
\hline 31 & $\ldots$ & $-\cdots$ & 55 & $-\cdots$ & 40 & 44 & & & & & ---- & 67 \\
\hline $\begin{array}{l}\mathrm{Avg} \\
\mathrm{Max} \\
\mathrm{Min}\end{array}$ & & $\begin{array}{l}64 \\
71 \\
58\end{array}$ & $\begin{array}{l}59 \\
62 \\
55\end{array}$ & $\begin{array}{l}53 \\
57 \\
47\end{array}$ & $\begin{array}{l}47 \\
52 \\
40\end{array}$ & $\begin{array}{l}41 \\
47 \\
37\end{array}$ & & & & & $\begin{array}{l}61 \\
66 \\
59\end{array}$ & $\begin{array}{l}67 \\
69 \\
63\end{array}$ \\
\hline
\end{tabular}


Table 21.--Monthly maximum, minimum, and mean water temperatures of Columbia River at Bonneville Dam forebay

\begin{tabular}{|c|c|c|c|c|c|c|c|c|c|c|c|c|}
\hline $\begin{array}{l}\text { Water } \\
\text { Year }\end{array}$ & Oct. & Nov. & Dec. & Jan. & Feb. & Mar. & Apr. & May & June & July & Aug. & Sept. \\
\hline 1938 & & & & & & & & $\begin{array}{r}54 \\
59 \quad 52 \\
\end{array}$ & $\begin{array}{r}59 \\
63 \quad 56 \\
\end{array}$ & $\begin{array}{r}67 \\
72 \quad 62 \\
\end{array}$ & $\begin{array}{c}68 \\
70 \quad 67 \\
\end{array}$ & $\begin{array}{r}68 \\
68 \quad 67 \\
\end{array}$ \\
\hline 1939 & $\begin{array}{r}59 \\
66 \quad 53 \\
\end{array}$ & $\begin{array}{r}47 \\
54 \quad 39 \\
\end{array}$ & $\begin{array}{r}39 \\
42 \quad 35 \\
\end{array}$ & $\begin{array}{r}40 \\
41 \quad 38 \\
\end{array}$ & $\begin{array}{r}39 \\
41 \quad 35 \\
\end{array}$ & $\begin{array}{r}45 \\
50 \quad 41 \\
\end{array}$ & $\begin{array}{r}51 \\
54 \quad 49 \\
\end{array}$ & $\begin{array}{rr}55 \\
57 \quad 54 \\
\end{array}$ & $\begin{array}{r}58 \\
63 \quad 56 \\
\end{array}$ & $\begin{array}{r}66 \\
71 \quad 63 \\
\end{array}$ & $\begin{array}{c}69 \\
70 \quad 67 \\
\end{array}$ & $\begin{array}{r}64 \\
66 \quad 62 \\
\end{array}$ \\
\hline 1940 & $\begin{array}{c}57 \\
63 \quad 52 \\
\end{array}$ & $\begin{array}{c}47 \\
52 \quad 43 \\
\end{array}$ & $\begin{array}{r}43 \\
44 \quad 39 \\
\end{array}$ & $\begin{array}{c}37 \\
40 \quad 34 \\
\end{array}$ & $\begin{array}{rr} & 39 \\
42 \quad 34 \\
\end{array}$ & $\begin{array}{rr}46 \\
51 \quad 42 \\
\end{array}$ & $\begin{array}{r}52 \\
54 \quad 49 \\
\end{array}$ & \begin{tabular}{rr}
\multicolumn{2}{|c}{57} \\
$61 \quad 53$ \\
\end{tabular} & $\begin{array}{c}63 \\
66 \quad 59 \\
\end{array}$ & $\begin{array}{c}68 \\
70 \quad 66 \\
\end{array}$ & $\begin{array}{c}69 \\
70 \quad 67 \\
\end{array}$ & $\begin{array}{r}67 \\
69 \quad 65 \\
\end{array}$ \\
\hline 1941 & $\begin{array}{r}60 \\
64 \quad 55 \\
\end{array}$ & $\begin{array}{r}46 \\
54 \quad 41 \\
\end{array}$ & $\begin{array}{r}40 \\
43 \quad 35 \\
\end{array}$ & $\begin{array}{c}37 \\
40 \quad 35 \\
\end{array}$ & $\begin{array}{r}40 \\
42 \quad 39 \\
\end{array}$ & $\begin{array}{cc}46 \\
52 \quad 42 \\
\end{array}$ & $\begin{array}{rr}53 \\
58 \quad 51 \\
\end{array}$ & $\begin{array}{rr} & 58 \\
60 \quad 55 \\
\end{array}$ & $\begin{array}{r}62 \\
65 \quad 58 \\
\end{array}$ & $\begin{array}{c}70 \\
75 \quad 64 \\
\end{array}$ & $\begin{array}{c}70 \\
74 \quad 67 \\
\end{array}$ & $\begin{array}{c}64 \\
67 \quad 61 \\
\end{array}$ \\
\hline 1942 & $\begin{array}{r}57 \\
62 \quad 54 \\
\end{array}$ & $\begin{array}{r}50 \\
54 \quad 44 \\
\end{array}$ & $\begin{array}{r}43 \\
47 \quad 39 \\
\end{array}$ & $\begin{array}{cc} & 34 \\
37 \quad 31 \\
\end{array}$ & $\begin{array}{r}39 \\
40 \quad 36 \\
\end{array}$ & $\begin{array}{r}42 \\
46 \quad 38 \\
\end{array}$ & $\begin{array}{c}51 \\
53 \quad 46 \\
\end{array}$ & \begin{tabular}{rr}
\multicolumn{2}{c}{54} \\
$58 \quad 49$ \\
\end{tabular} & $\begin{array}{r}58 \\
62 \quad 56 \\
\end{array}$ & $\begin{array}{c}65 \\
68 \quad 61 \\
\end{array}$ & $\begin{array}{r}69 \\
71 \quad 66 \\
\end{array}$ & $\begin{array}{r}65 \\
68 \quad 62 \\
\end{array}$ \\
\hline 1943 & $\begin{array}{r}59 \\
65 \quad 54 \\
\end{array}$ & $\begin{array}{r}47 \\
53 \quad 44 \\
\end{array}$ & $\begin{array}{r}42 \\
44 \quad 40 \\
\end{array}$ & $\begin{array}{r}37 \\
41 \quad 32 \\
\end{array}$ & $\begin{array}{r}39 \\
42 \quad 33 \\
\end{array}$ & $\begin{array}{r}43 \\
48 \quad 41 \\
\end{array}$ & $\begin{array}{r}49 \\
53 \quad 46 \\
\end{array}$ & $\begin{array}{c}52 \\
57 \quad 50 \\
\end{array}$ & $\begin{array}{r}56 \\
60 \quad 53 \\
\end{array}$ & $\begin{array}{c}63 \\
66 \quad 60 \\
\end{array}$ & $\begin{array}{c}66 \\
68 \quad 65 \\
\end{array}$ & $\begin{array}{c}65 \\
66 \quad 63 \\
\end{array}$ \\
\hline 1944 & $\begin{array}{rr} & 58 \\
64 \quad 51 \\
\end{array}$ & $\begin{array}{c}47 \\
52 \quad 43 \\
\end{array}$ & $\begin{array}{c}41 \\
46 \quad 38 \\
\end{array}$ & $\begin{array}{r}38 \\
41 \quad 35 \\
\end{array}$ & $\begin{array}{r}40 \\
42 \quad 37 \\
\end{array}$ & $\begin{array}{r}42 \\
45 \quad 40 \\
\end{array}$ & $\begin{array}{cc} & 50 \\
53 \quad 44 \\
\end{array}$ & $\begin{array}{rr}55 \\
58 \quad 52 \\
\end{array}$ & $\begin{array}{rr}59 \\
63 \quad 57 \\
\end{array}$ & $\begin{array}{c}66 \\
70 \quad 63 \\
\end{array}$ & $\begin{array}{c}68 \\
70 \quad 66 \\
\end{array}$ & $\begin{array}{r}65 \\
69 \quad 61 \\
\end{array}$ \\
\hline 1945 & $\begin{array}{r}60 \\
64 \quad 57 \\
\end{array}$ & $\begin{array}{rr}50 \\
57 \quad 43 \\
\end{array}$ & $\begin{array}{r}40 \\
44 \quad 37 \\
\end{array}$ & $\begin{array}{c}41 \\
45 \quad 37 \\
\end{array}$ & $\begin{array}{c}41 \\
45 \quad 37 \\
\end{array}$ & $\begin{array}{r}43 \\
48 \quad 40 \\
\end{array}$ & $\begin{array}{r}49 \\
54 \quad 47 \\
\end{array}$ & $\begin{array}{rr} & 56 \\
60 \quad 53 \\
\end{array}$ & $\begin{array}{r}59 \\
64 \quad 56 \\
\end{array}$ & $\begin{array}{r}66 \\
69 \quad 61 \\
\end{array}$ & $\begin{array}{r}69 \\
71 \quad 67 \\
\end{array}$ & $\begin{array}{r}65 \\
69 \quad 59 \\
\end{array}$ \\
\hline 1946 & $\begin{array}{rr} & 59 \\
62 \quad 52 \\
\end{array}$ & $\begin{array}{c}47 \\
54 \quad 44 \\
\end{array}$ & $\begin{array}{r}40 \\
44 \quad 37 \\
\end{array}$ & $\begin{array}{r}40 \\
42 \quad 39 \\
\end{array}$ & $\begin{array}{r}40 \\
42 \quad 39 \\
\end{array}$ & $\begin{array}{c}44 \\
46 \quad 42 \\
\end{array}$ & $\begin{array}{rr}49 \\
52 \quad 45 \\
\end{array}$ & $\begin{array}{c}53 \\
56 \quad 50 \\
\end{array}$ & $\begin{array}{r}58 \\
61 \quad 55 \\
\end{array}$ & $\begin{array}{c}64 \\
68 \quad 60 \\
\end{array}$ & $\begin{array}{c}68 \\
70 \quad 66 \\
\end{array}$ & $\begin{array}{r}65 \\
69 \quad 60 \\
\end{array}$ \\
\hline 1947 & $\begin{array}{rr}55 \\
60 \quad 50 \\
\end{array}$ & $\begin{array}{r}45 \\
50 \quad 42 \\
\end{array}$ & $\begin{array}{r}42 \\
48 \quad 39 \\
\end{array}$ & $\begin{array}{r}37 \\
42 \quad 34 \\
\end{array}$ & $\begin{array}{r}40 \\
44 \quad 38 \\
\end{array}$ & $\begin{array}{r}45 \\
49 \quad 42 \\
\end{array}$ & $\begin{array}{r}50 \\
54 \quad 47 \\
\end{array}$ & $\begin{array}{rr} & 55 \\
60 \quad 53 \\
\end{array}$ & $\begin{array}{r}59 \\
62 \quad 57 \\
\end{array}$ & $\begin{array}{r}65 \\
68 \quad 61 \\
\end{array}$ & $\begin{array}{r}67 \\
69 \quad 65 \\
\end{array}$ & $\begin{array}{r}64 \\
68 \quad 61 \\
\end{array}$ \\
\hline 1948 & $\begin{array}{rr}60 \\
65 \quad 56 \\
\end{array}$ & $\begin{array}{c}51 \\
57 \quad 47 \\
\end{array}$ & $\begin{array}{r}45 \\
48 \quad 44 \\
\end{array}$ & $\begin{array}{r}41 \\
44 \quad 39 \\
\end{array}$ & $\begin{array}{r}40 \\
44 \quad 37 \\
\end{array}$ & $\begin{array}{r}44 \\
48 \quad 43 \\
\end{array}$ & $\begin{array}{rr}50 \\
53 \quad 47 \\
\end{array}$ & $\begin{array}{r}52 \\
55 \quad 48 \\
\end{array}$ & $\begin{array}{cc} & 55 \\
57 \quad 53 \\
\end{array}$ & $\begin{array}{rr} & 59 \\
61 \quad 57 \\
\end{array}$ & $\begin{array}{c}63 \\
64 \quad 61 \\
\end{array}$ & $\begin{array}{r}63 \\
65 \quad 59 \\
\end{array}$ \\
\hline 1949 & $\begin{array}{r}57 \\
59 \quad 55 \\
\end{array}$ & $\begin{array}{c}50 \\
54 \quad 47 \\
\end{array}$ & $\begin{array}{r}43 \\
47 \quad 39 \\
\end{array}$ & $\begin{array}{r}36 \\
38 \quad 32 \\
\end{array}$ & $\begin{array}{cc} & 34 \\
40 \quad 32 \\
\end{array}$ & $\begin{array}{r}43 \\
46 \quad 40 \\
\end{array}$ & $\begin{array}{cc} & 49 \\
51 \quad 45 \\
\end{array}$ & $\begin{array}{rr}52 \\
54 \quad 49 \\
\end{array}$ & $\begin{array}{c}56 \\
57 \quad 50 \\
\end{array}$ & $\begin{array}{c}61 \\
65 \quad 57 \\
\end{array}$ & $\begin{array}{r}67 \\
71 \quad 65 \\
\end{array}$ & $\begin{array}{c}65 \\
70 \quad 61 \\
\end{array}$ \\
\hline 1950 & $\begin{array}{rr}54 \\
63 \quad 49 \\
\end{array}$ & $\begin{array}{r}50 \\
52 \quad 47 \\
\end{array}$ & $\begin{array}{r}42 \\
49 \quad 39 \\
\end{array}$ & $\begin{array}{r}34 \\
40 \quad 32 \\
\end{array}$ & $\begin{array}{rr}35 \\
41 \quad 32 \\
\end{array}$ & $\begin{array}{r}42 \\
44 \quad 40 \\
\end{array}$ & $\begin{array}{r}47 \\
49 \quad 44 \\
\end{array}$ & $\begin{array}{rr}52 \\
55 \quad 49 \\
\end{array}$ & $\begin{array}{c}55 \\
57 \quad 54 \\
\end{array}$ & $\begin{array}{c}62 \\
66 \quad 58 \\
\end{array}$ & $\begin{array}{c}68 \\
71 \quad 61 \\
\end{array}$ & $\begin{array}{r}66 \\
69 \quad 63 \\
\end{array}$ \\
\hline 1951 & $\begin{array}{r}57 \\
63 \quad 51 \\
\end{array}$ & $\begin{array}{r}48 \\
52 \quad 45 \\
\end{array}$ & $\begin{array}{r}43 \\
45 \quad 41 \\
\end{array}$ & $\begin{array}{r}37 \\
44 \quad 34 \\
\end{array}$ & $\begin{array}{rr}38 \\
41 \quad 32 \\
\end{array}$ & $\begin{array}{c}41 \\
46 \quad 38 \\
\end{array}$ & $\begin{array}{cc} & 49 \\
52 \quad 46 \\
\end{array}$ & $\begin{array}{rr}53 \\
55 \quad 49 \\
\end{array}$ & $\begin{array}{c}57 \\
60 \quad 54 \\
\end{array}$ & $\begin{array}{c}64 \\
66 \quad 61 \\
\end{array}$ & $\begin{array}{c}67 \\
70 \quad 66 \\
\end{array}$ & $\begin{array}{c}65 \\
66 \quad 62 \\
\end{array}$ \\
\hline 1952 & $\begin{array}{r}58 \\
62 \quad 52 \\
\end{array}$ & $\begin{array}{r}48 \\
51 \quad 45 \\
\end{array}$ & $\begin{array}{r}41 \\
45 \quad 35 \\
\end{array}$ & $\begin{array}{rr} & 36 \\
38 \quad 34 \\
\end{array}$ & $\begin{array}{r}39 \\
40 \quad 37 \\
\end{array}$ & $\begin{array}{r}42 \\
47 \quad 40 \\
\end{array}$ & $\begin{array}{rr} & 50 \\
54 \quad 46 \\
\end{array}$ & $\begin{array}{rr} & 54 \\
57 \quad 52 \\
\end{array}$ & $\begin{array}{r}59 \\
62 \quad 56 \\
\end{array}$ & $\begin{array}{c}65 \\
67 \quad 61 \\
\end{array}$ & $\begin{array}{c}68 \\
70 \quad 66 \\
\end{array}$ & $\begin{array}{r}65 \\
66 \quad 61 \\
\end{array}$ \\
\hline 1.953 & $\begin{array}{c}61 \\
65 \quad 57 \\
\end{array}$ & $\begin{array}{cc} & 48 \\
57 \quad 40 \\
\end{array}$ & $\begin{array}{r}42 \\
44 \quad 40 \\
\end{array}$ & $\begin{array}{r}43 \\
45 \quad 40 \\
\end{array}$ & $\begin{array}{r}44 \\
45 \quad 42 \\
\end{array}$ & $\begin{array}{r}46 \\
48 \quad 42 \\
\end{array}$ & $\begin{array}{r}50 \\
55 \quad 47 \\
\end{array}$ & $\begin{array}{rr}55 \\
58 \quad 52 \\
\end{array}$ & $\begin{array}{c}57 \\
60 \quad 53 \\
\end{array}$ & $\begin{array}{c}64 \\
66 \quad 60 \\
\end{array}$ & $\begin{array}{c}68 \\
70 \quad 66 \\
\end{array}$ & $\begin{array}{c}66 \\
68 \quad 61 \\
\end{array}$ \\
\hline
\end{tabular}




\begin{tabular}{|c|c|c|c|c|c|c|c|c|c|c|c|c|}
\hline 1954 & $\begin{array}{r}58 \\
6156\end{array}$ & $\begin{array}{c}52 \\
56 \quad 49\end{array}$ & $\begin{array}{r}46 \\
49 \quad 43\end{array}$ & $\begin{array}{c}40 \\
4336\end{array}$ & $\begin{array}{c}40 \\
44 \quad 37\end{array}$ & $\begin{array}{c}44 \\
45 \quad 42\end{array}$ & $\begin{array}{c}49 \\
52 \quad 44\end{array}$ & $\begin{array}{c}54 \\
56 \quad 50\end{array}$ & $\begin{array}{c}56 \\
5954\end{array}$ & $\begin{array}{c}61 \\
6458\end{array}$ & $\begin{array}{c}64 \\
6663\end{array}$ & 65 \\
\hline 1955 & $\begin{array}{c}56 \\
60 \quad 52\end{array}$ & $\begin{array}{c}51 \\
5546\end{array}$ & $\begin{array}{c}42 \\
46 \quad 39\end{array}$ & $\begin{array}{r}39 \\
41 \quad 38\end{array}$ & $\begin{array}{c}39 \\
4139\end{array}$ & $\begin{array}{c}40 \\
4237\end{array}$ & $\begin{array}{c}46 \\
47 \quad 43\end{array}$ & $\begin{array}{c}52 \\
56 \quad 49\end{array}$ & $\begin{array}{c}57 \\
5954\end{array}$ & $\begin{array}{c}61 \\
6457\end{array}$ & $\begin{array}{c}66 \\
6864\end{array}$ & $\begin{array}{c}65 \\
6960\end{array}$ \\
\hline 1956 & $\begin{array}{r}57 \\
60 \quad 53\end{array}$ & $\begin{array}{r}45 \\
5339\end{array}$ & $\begin{array}{r}40 \\
43 \quad 36\end{array}$ & $\begin{array}{c}39 \\
40 \quad 35\end{array}$ & $\begin{array}{c}34 \\
36 \quad 32\end{array}$ & $\begin{array}{c}42 \\
46 \quad 37 \\
\end{array}$ & $\begin{array}{c}48 \\
5246\end{array}$ & $\begin{array}{r}53 \\
5750\end{array}$ & $\begin{array}{c}57 \\
60 \quad 55\end{array}$ & $\begin{array}{c}64 \\
68 \quad 55\end{array}$ & $\begin{array}{r}68 \\
71 \quad 64\end{array}$ & $\begin{array}{c}66 \\
6864\end{array}$ \\
\hline 1957 & $\begin{array}{r}58 \\
6352 \\
\end{array}$ & $\begin{array}{r}48 \\
5242 \\
\end{array}$ & $\begin{array}{r}42 \\
44 \quad 40 \\
\end{array}$ & $\begin{array}{r}37 \\
40 \quad 32 \\
\end{array}$ & $\begin{array}{r}36 \\
36 \quad 32 \\
\end{array}$ & $\begin{array}{r}44 \\
47 \quad 40 \\
\end{array}$ & $\begin{array}{r}50 \\
54 \quad 47 \\
\end{array}$ & $\begin{array}{r}55 \\
58 \quad 52 \\
\end{array}$ & $\begin{array}{r}60 \\
63 \quad 58 \\
\end{array}$ & $\begin{array}{r}66 \\
6862 \\
\end{array}$ & $\begin{array}{r}68 \\
6967 \\
\end{array}$ & $\begin{array}{r}67 \\
6965 \\
\end{array}$ \\
\hline 1958 & $\begin{array}{c}59 \\
65 \quad 55\end{array}$ & $\begin{array}{c}51 \\
56^{46}\end{array}$ & $\begin{array}{c}44 \\
47 \quad 42\end{array}$ & $\begin{array}{c}41 \\
4340\end{array}$ & $\begin{array}{r}45 \\
4743\end{array}$ & $\begin{array}{c}45 \\
4843\end{array}$ & $\begin{array}{c}50 \\
5248\end{array}$ & $\begin{array}{c}57 \\
60 \quad 52\end{array}$ & $\begin{array}{c}63 \\
6860\end{array}$ & $\begin{array}{c}69 \\
7464\end{array}$ & $\begin{array}{c}72 \\
7370\end{array}$ & $\begin{array}{c}66 \\
70 \quad 62\end{array}$ \\
\hline 1959 & $\begin{array}{r}60 \\
63 \quad 55\end{array}$ & $\begin{array}{c}50 \\
55 \quad 42\end{array}$ & $\begin{array}{c}43 \\
45 \quad 41\end{array}$ & $\begin{array}{r}40 \\
44 \quad 38 \\
\end{array}$ & $\begin{array}{c}41 \\
4239\end{array}$ & $\begin{array}{c}44 \\
45 \quad 41\end{array}$ & $\begin{array}{c}49 \\
5246\end{array}$ & $\begin{array}{r}53 \\
56 \quad 50 \\
\end{array}$ & $\begin{array}{r}58 \\
60 \quad 52\end{array}$ & $\begin{array}{c}64 \\
6860\end{array}$ & $\begin{array}{r}67 \\
6865\end{array}$ & $\begin{array}{c}64 \\
6660\end{array}$ \\
\hline 1960 & $\begin{array}{r}58 \\
60 \quad 55 \\
\end{array}$ & $\begin{array}{r}48 \\
55 \quad 44 \\
\end{array}$ & $\begin{array}{r}43 \\
46 \quad 41 \\
\end{array}$ & $\begin{array}{r}37 \\
41 \quad 34 \\
\end{array}$ & $\begin{array}{c}40 \\
41 \quad 37 \\
\end{array}$ & $48 \quad 36$ & $\begin{array}{c}48 \\
51 \quad 46\end{array}$ & $\begin{array}{c}54 \\
5852\end{array}$ & $\begin{array}{c}59 \\
6357\end{array}$ & $\begin{array}{c}66 \\
6963\end{array}$ & $\begin{array}{c}68 \\
70 \quad 64\end{array}$ & $\begin{array}{c}65 \\
6764\end{array}$ \\
\hline 1961 & $\begin{array}{c}60 \\
66 \quad 56\end{array}$ & $\begin{array}{c}51 \\
5646\end{array}$ & $\begin{array}{c}42 \\
48 \quad 40 \\
\end{array}$ & $\begin{array}{c}41 \\
4238 \\
\end{array}$ & $\begin{array}{r}43 \\
44 \quad 41 \\
\end{array}$ & $\begin{array}{r}45 \\
48 \quad 42 \\
\end{array}$ & $\begin{array}{c}50 \\
52 \quad 48 \\
\end{array}$ & $\begin{array}{r}55 \\
58 \quad 52 \\
\end{array}$ & $\begin{array}{r}60 \\
63 \quad 55\end{array}$ & $\begin{array}{c}67 \\
7061\end{array}$ & $\begin{array}{c}71 \\
7269\end{array}$ & $\begin{array}{c}65 \\
7060\end{array}$ \\
\hline 1962 & $\begin{array}{c}58 \\
6152\end{array}$ & $\begin{array}{c}48 \\
53 \quad 44\end{array}$ & $\begin{array}{c}42 \\
44 \quad 40\end{array}$ & $\begin{array}{r}39 \\
44 \quad 34\end{array}$ & $\begin{array}{c}40 \\
4237\end{array}$ & $\begin{array}{c}42 \\
46 \quad 38\end{array}$ & $\begin{array}{c}50 \\
5346\end{array}$ & $\begin{array}{c}53 \\
5650\end{array}$ & $\begin{array}{c}59 \\
6355\end{array}$ & $\begin{array}{r}65 \\
70 \quad 62\end{array}$ & $\begin{array}{r}69 \\
7166\end{array}$ & $\begin{array}{c}66 \\
68 \quad 64\end{array}$ \\
\hline 1963 & $\begin{array}{r}59 \\
64 \quad 56 \\
\end{array}$ & $\begin{array}{r}53 \\
5647 \\
\end{array}$ & $\begin{array}{r}46 \\
48 \quad 44\end{array}$ & $\begin{array}{r}39 \\
44 \quad 34 \\
\end{array}$ & $\begin{array}{r}40 \\
43 \quad 35\end{array}$ & $\begin{array}{r}45 \\
46 \quad 43 \\
\end{array}$ & $\begin{array}{c}49 \\
51 \quad 47\end{array}$ & $\begin{array}{r}56 \\
60 \quad 50\end{array}$ & $\begin{array}{r}61 \\
6358\end{array}$ & $\begin{array}{r}65 \\
6762\end{array}$ & $\begin{array}{r}69 \\
7066\end{array}$ & $\begin{array}{c}68 \\
70 \quad 66\end{array}$ \\
\hline 1964 & $\begin{array}{c}62 \\
6756\end{array}$ & $\begin{array}{c}52 \\
5648\end{array}$ & $\begin{array}{c}43 \\
4641\end{array}$ & $\begin{array}{c}42 \\
44 \quad 40\end{array}$ & $\begin{array}{r}42 \\
44 \quad 40\end{array}$ & $\begin{array}{c}44 \\
46 \quad 42\end{array}$ & $\begin{array}{c}49 \\
51 \quad 46\end{array}$ & $\begin{array}{c}54 \\
57 \quad 50\end{array}$ & $\begin{array}{r}57 \\
60 \quad 55\end{array}$ & $\begin{array}{c}64 \\
6760\end{array}$ & $\begin{array}{c}66 \\
6963\end{array}$ & $\begin{array}{c}63 \\
6462\end{array}$ \\
\hline 1965 & $\begin{array}{r}58 \\
63 \quad 50 \\
\end{array}$ & $\begin{array}{r}49 \\
55 \quad 43 \\
\end{array}$ & $\begin{array}{r}41 \\
47 \quad 35 \\
\end{array}$ & $\begin{array}{r}39 \\
42 \quad 37 \\
\end{array}$ & $\begin{array}{r}41 \\
43 \quad 40 \\
\end{array}$ & $\begin{array}{r}43 \\
44 \quad 41 \\
\end{array}$ & $\begin{array}{r}49 \\
53 \quad 44 \\
\end{array}$ & $\begin{array}{r}54 \\
57 \quad 50 \\
\end{array}$ & $\begin{array}{r}59 \\
6157\end{array}$ & $\begin{array}{r}65 \\
6961\end{array}$ & $\begin{array}{r}70 \\
7265 \\
\end{array}$ & $\begin{array}{c}64 \\
66 \quad 62 \\
\end{array}$ \\
\hline 1966 & $\begin{array}{c}60 \\
6258\end{array}$ & $\begin{array}{r}53 \\
5848\end{array}$ & $\begin{array}{c}45 \\
48 \quad 42\end{array}$ & $\begin{array}{c}41 \\
4239\end{array}$ & $\begin{array}{c}41 \\
4239\end{array}$ & $\begin{array}{c}44 \\
48 \quad 41\end{array}$ & $\begin{array}{c}51 \\
5348\end{array}$ & $\begin{array}{c}56 \\
5853\end{array}$ & $\begin{array}{r}59 \\
6156\end{array}$ & $\begin{array}{c}64 \\
66 \quad 58\end{array}$ & $\begin{array}{c}67 \\
7066\end{array}$ & $\begin{array}{c}66 \\
68 \quad 65\end{array}$ \\
\hline
\end{tabular}


